\title{
UNKNOTTED REEB ORBITS AND NICELY EMBEDDED HOLOMORPHIC CURVES
}

\author{
ALEXANDRU CIOBA AND CHRIS WENDL
}

\begin{abstract}
We exhibit a distinctly low-dimensional dynamical obstruction to the existence of Liouville cobordisms: for any contact 3-manifold admitting an exact symplectic cobordism to the tight 3-sphere, every nondegenerate contact form admits an embedded Reeb orbit that is unknotted and has self-linking number -1 . The same is true moreover for any contact structure on a closed 3-manifold that is reducible. Our results generalize an earlier theorem of Hofer-Wysocki-Zehnder for the 3-sphere, but use somewhat newer techniques: the main idea is to exploit the intersection theory of punctured holomorphic curves in order to understand the compactification of the space of so-called "nicely embedded" curves in symplectic cobordisms. In the process, we prove a local adjunction formula for holomorphic annuli breaking along a Reeb orbit, which may be of independent interest.
\end{abstract}

\section{Contents}

1. Introduction

1.1. Statement of the main results

1.2. Context

1.3. Applications

1.4. Outline of proofs, part 1: seed curves and compactness

1.5. Outline of proofs, part 2: intersections

1.6. Local adjunction

1.7. Outline of proofs, conclusion

1.8. Acknowledgements

2. Preparation

2.1. Contact manifolds and symplectic cobordisms

2.2. Reeb orbits and the Conley-Zehnder index

2.3. Holomorphic curves in completed symplectic cobordisms

2.4. The low-dimensional case

3. Seed curves in the positive end

3.1. The standard sphere

3.2. Reducible tight contact 3-manifolds

3.3. Overtwisted contact 3-manifolds

4. A local adjunction formula for breaking holomorphic annuli

5. Compactness for nicely embedded planes in cobordisms

6. Proofs of the main theorems

Appendix A. Liouville cobordisms from exact Lagrangian caps References 


\section{INTRODUCTION}

1.1. Statement of the main results. Contact structures arise in the context of Hamiltonian dynamics via the notion of convexity: a convex hypersurface in a symplectic manifold naturally inherits a contact structure, and the orbits of its Reeb vector field then match the Hamiltonian orbits defined by any Hamiltonian function that has the hypersurface as a regular level set. In this paper, we consider contact structures that are induced on convex and concave boundaries of symplectic manifolds, i.e. symplectic cobordisms. Our main theorem relates the existence of exact symplectic cobordisms between given contact manifolds to a dynamical condition on their Reeb vector fields. In particular, we will restrict attention to dimension three and discuss the existence of closed Reeb orbits $\gamma: S^{1} \rightarrow M$ that are not only contractible but also unknotted, meaning

$$
\gamma=\left.f\right|_{\partial \mathbb{D}^{2}} \quad \text { for some embedding } f: \mathbb{D}^{2} \hookrightarrow M,
$$

where $\mathbb{D}^{2} \subset \mathbb{C}$ denotes the closed unit disk. All definitions relevant to the following statements may be found in 92.1 , but let us stress the following convention from the start since it sometimes causes confusion:

Convention. In this paper, the words "symplectic cobordism from $\left(M_{1}, \xi_{1}\right)$ to $\left(M_{2}, \xi_{2}\right)$ " always mean that $\left(M_{1}, \xi_{1}\right)$ is the concave boundary and $\left(M_{2}, \xi_{2}\right)$ the convex boundary of the cobordism (cf. \$2.1). This usage is standard, and is natural from the perspective of contact surgery, but a few other authors (especially e.g. in the literature on embedded contact homology) sometimes interchange the order of "convex" and "concave," which would make our results false.

Theorem 1.1. Assume $(M, \xi)$ is a closed contact 3-manifold that admits a Liouville cobordism to the standard contact 3 -sphere $\left(S^{3}, \xi_{\text {std }}\right)$. Then for every nondegenerate contact form $\alpha$ on $(M, \xi)$, the Reeb vector field $R_{\alpha}$ admits a simple closed orbit $\gamma$ whose image is the boundary of an embedded disk $\mathcal{D} \subset M$. Moreover, the Conley-Zehnder index and self-linking number of $\gamma$ with respect to $\mathcal{D}$ satisfy

$$
\mu_{\mathrm{CZ}}(\gamma ; \mathcal{D}) \in\{2,3\} \quad \text { and } \quad \operatorname{sl}(\gamma ; \mathcal{D})=-1 .
$$

A minor variation on the same techniques in the spirit of [Hof93] will also imply the following:

Theorem 1.2. Assume $(M, \xi)$ is a closed contact 3-manifold and that either of the following is true:

(1) $M$ is reducible, i.e. it contains an embedded 2-sphere that does not bound an embedded ball;

(2) $(M, \xi)$ admits a Liouville cobordism to an overtwisted contact manifold.

Then for every nondegenerate contact form $\alpha$ on $(M, \xi)$, the Reeb vector field $R_{\alpha}$ admits a simple closed orbit $\gamma$ whose image is the boundary of an embedded disk $\mathcal{D} \subset M$ such that

$$
\mu_{\mathrm{CZ}}(\gamma ; \mathcal{D})=2 \quad \text { and } \quad \operatorname{sl}(\gamma ; \mathcal{D})=-1 .
$$


Recall that an oriented 3-manifold is reducible if and only if it is either $S^{1} \times S^{2}$ or $M_{1} \# M_{2}$ for a pair of closed oriented 3-manifolds that are not spheres. This condition is now known to be equivalent to the hypothesis $\pi_{2}(M) \neq 0$ used in Hof93: in one direction this follows from the sphere theorem for 3-manifolds, and in the other, from [Hat, Prop. 3.10] and the Poincaré conjecture. Note that both of the above theorems require nondegeneracy of the contact form $\alpha$, but it is possible for the sake of applications to weaken this condition; see Theorem 1.12 below.

1.2. Context. The prototype for Theorems 1.1 and 1.2 is a 20-year-old result of Hofer-Wysocki-Zehnder HWZ96c, which amounts to the case $(M, \xi)=\left(S^{3}, \xi_{\text {std }}\right)$ of Theorem 1.1. The result in HWZ96c was in some sense far ahead of its time, as it required ideas from both the compactness theory $\left[\mathrm{BEH}^{+} 03\right]$ and the intersection theory [Sie11] of punctured holomorphic curves, but it appeared several years before either of those theories were developed in earnest. In the mean time the available techniques have improved, and our proofs will make use of those improvements.

A weaker version of Theorem 1.1 can be shown to hold in all dimensions, namely:

Theorem 1.3. If $(M, \xi)$ is a closed $(2 n-1)$-dimensional contact manifold admitting a Liouville cobordism to a standard contact sphere $\left(S^{2 n-1}, \xi_{\mathrm{std}}\right)$, then every contact form for $(M, \xi)$ admits a contractible closed Reeb orbit.

This result can largely be attributed to Hofer, as most of the ideas needed for its proof are present in Hof93. We will sketch a proof in $\$ 1.4$ which is similar in spirit to one that has previously appeared in the work of Geiges and Zehmisch [GZ12, Corollary 3.3] (see also [GZ13a, GZ13b); there is also an alternative proof via symplectic homology by Albers, Cieliebak and Oancea (see the appendix of [CO18]). Analogous results that may be viewed as higher-dimensional versions of Theorem 1.2 have appeared in AH09, NR11, GZ16, GNW16. The conclusions of our main results however are stronger and uniquely low dimensional: for instance in $\$ 1.3$ below, we will see examples of contact 3-manifolds that always admit contractible but not necessarily unknotted Reeb orbits. Theorem 1.1 thus gives a new means of proving that these examples cannot be exactly cobordant to the standard 3 -sphere.

We are aware of three general classes of contact 3-manifolds that satisfy the hypothesis of Theorem 1.1.

Example 1.4. If $\xi$ is overtwisted, then a theorem of Etnyre and Honda EH02 provides Stein cobordisms from $(M, \xi)$ to any other contact 3-manifold, so in particular to $\left(S^{3}, \xi_{\text {std }}\right)$. Of course, in this case Theorem 1.2 also applies and gives a slightly stronger result.

Example 1.5. Suppose $(M, \xi)$ is subcritically Stein fillable, or equivalently, that it can be obtained by performing contact connected sums on copies of the tight $S^{3}$ and $S^{1} \times S^{2}$. In this case, $(M, \xi)$ is the convex boundary of a Weinstein domain $W$ constructed by attaching 1-handles to a ball, and these 1-handles can then be cancelled by attaching suitable Weinstein 2-handles. This procedure embeds $W$ into the standard 4-ball as a Weinstein subdomain 
and thus produces a Weinstein cobordism from $(M, \xi)$ to $\left(S^{3}, \xi_{\text {std }}\right)$. Note that Theorem 1.2 also applies in this case unless $M=S^{3}$.

The third class of examples was brought to our attention by Emmy Murphy.

Example 1.6. Suppose $L \subset[1, \infty) \times S^{3}$ is an exact Lagrangian cap for some Legendrian knot $\Lambda$ in $\left(S^{3}, \xi_{\text {std }}\right)$, i.e. $L$ is a compact Lagrangian submanifold properly embedded in the top half of the symplectization $\mathbb{R} \times S^{3}$, such that $\partial L=\{1\} \times \Lambda, L$ is tangent near its boundary to a globally defined Liouville vector field pointing transversely inward at $\{1\} \times S^{3}$, and the restriction of the corresponding Liouville form to $L$ is exact. A result of Francesco Lin [Lin16] guarantees that such caps always exist after stabilizing $\Lambda$ sufficiently many times. Now suppose $\mathcal{U}_{L}$ is an open neighbourhood of $L$ in $[1, \infty) \times$ $S^{3}$, where the latter is viewed as sitting on top of the standard Weinstein filling $B^{4}$ of $\left(S^{3}, \xi_{\text {std }}\right)$. This neighbourhood can be choosen such that, after smoothing corners, $B^{4} \cup \overline{\mathcal{U}}_{L}$ is a Weinstein filling of some contact 3-manifold $(M, \xi)$, and $\left([1, T] \times S^{3}\right) \backslash \mathcal{U}_{L}$ for suitable $T>1$ defines a Liouville cobordism $W_{+}$from $(M, \xi)$ to $\left(S^{3}, \xi_{\text {std }}\right)$, see Figure1. Using a Morse function on $L$ that has one index 2 critical point and an inward gradient at $\partial L$, one can find a Weinstein handle decomposition of $B^{4} \cup \overline{\mathcal{U}}_{L}$ having exactly one 2-handle (see Remark A.2), thus $B^{4} \cup \overline{\mathcal{U}}_{L}$ is not subcritical, and it follows from the uniqueness of Stein fillings in the subcritical case [CE12, Theorem 16.9(c)] that $(M, \xi)$ is not subcritically fillable. For more details on this construction, see Appendix A.

One can now use a well-known result of Eliashberg [Eli90,CE12] to extract from this example contact 3 -manifolds other than $\left(S^{3}, \xi_{\text {std }}\right)$ to which Theorem 1.1 applies but Theorem 1.2 does not. Indeed, while $(M, \xi)=\partial\left(B^{4} \cup \overline{\mathcal{U}}_{L}\right)$ could be reducible, it is Stein fillable and therefore tight, so Colin [Col97. (see also [Gei08, §4.12]) provides a prime decomposition

$$
(M, \xi)=\left(M_{1}, \xi_{1}\right) \# \ldots \#\left(M_{k}, \xi_{k}\right),
$$

and Eliashberg's theorem implies that $B^{4} \cup \overline{\mathcal{U}}_{L}$ must be Weinstein deformation equivalent to a domain obtained by attaching Weinstein 1-handles to Weinstein fillings of the summands. But the summands cannot all be $S^{1} \times S^{2}$ since $(M, \xi)$ is not subcritically fillable, so at least one of them is an irreducible tight contact 3 -manifold admitting a Liouville cobordism to $\left(S^{3}, \xi_{\text {std }}\right)$.

Corollary 1.7. The contact 3-manifolds $(M, \xi)$ described in Example 1.6 and their prime summands all admit unknotted Reeb orbits with ConleyZehnder index 2 or 3 and self-linking number -1 for every choice of nondegenerate contact form.

The construction outlined in Example1.6 also works in higher dimensions using the exact Lagrangian caps of Eliashberg-Murphy EM13, cf. Appendix A. In this case it produces Weinstein subdomains of the standard ball which are presumably flexible in the sense of CE12. Recently, Murphy and Siegel [MS18] have also found examples of nonflexible Weinstein subdomains in the standard ball, whose boundaries therefore also satisfy the hypothesis of Theorem 1.3 . 


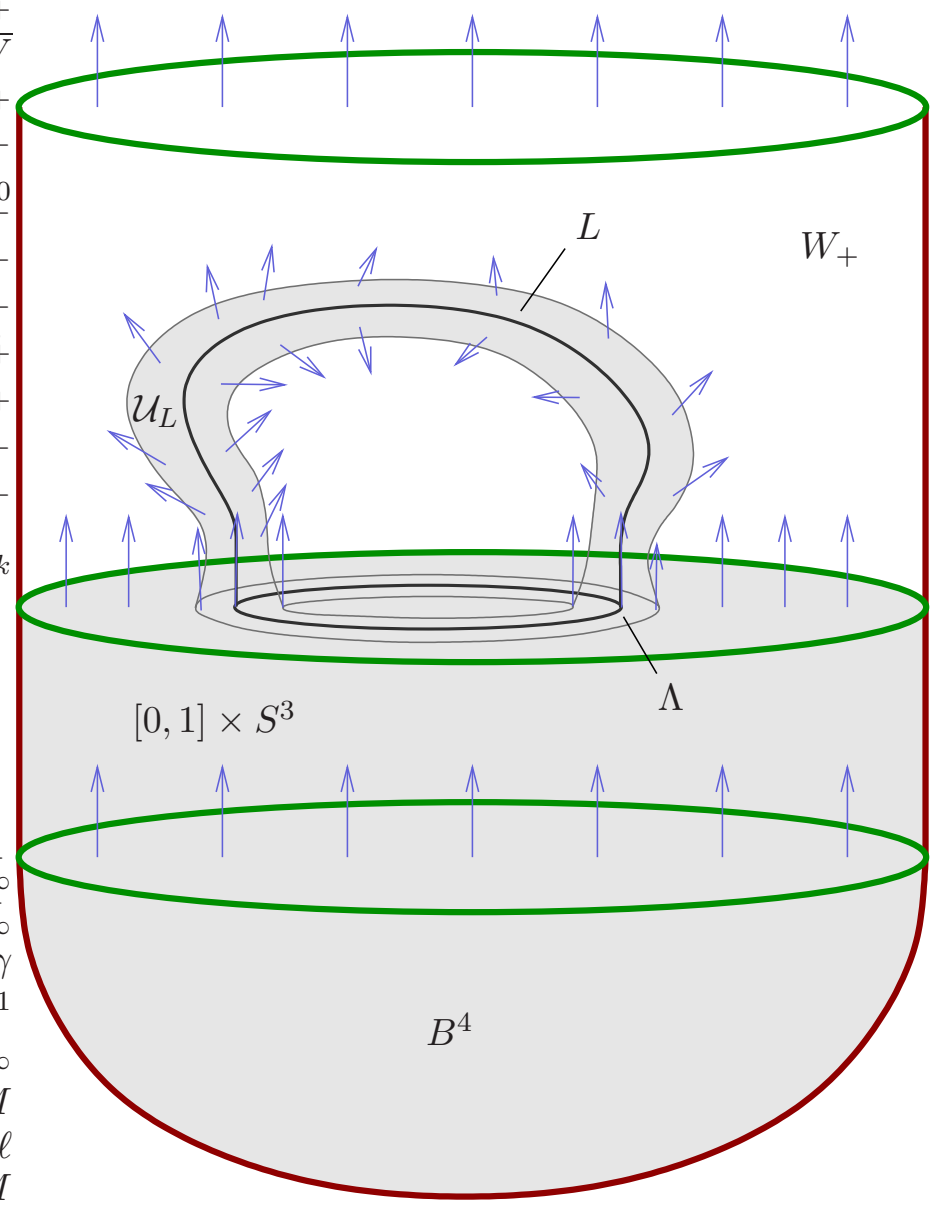

Figure 1. An exact Lagrangian cap for a Legendrian in $\left(S^{3}, \xi_{\text {std }}\right)$ produces a Liouville cobordism $W_{+}$from $(M, \xi)$ to $\left(S^{3}, \xi_{\text {std }}\right)$, where $(M, \xi):=\partial\left(B^{4} \cup \overline{\mathcal{U}}_{L}\right)$ is not subcritically fillable.

Remark 1.8. It is not known whether any contact 3-manifolds satisfy the hypothesis of Theorem 1.2(2) without being overtwisted, though Andy Wand Wan15 has proved that the answer is no under the stronger condition that the cobordism is Stein. Theorem 1.2(2) may thus be interpreted as a small measure of support for the conjecture that Wand's theorem extends to Liouville cobordisms (cf. Wena, Question 5]): that is, Theorem 1.2(2) provides a mechanism for detecting tightness, but it cannot detect the (conjecturally nonexistent) distinction between an overtwisted contact manifold and one that is only Liouville cobordant to something overtwisted.

We remark that the word "Liouville" definitely cannot be dropped from the statements of any of the above theorems: for instance, any Lagrangian torus in the standard symplectic $\mathbb{R}^{2 n}$ gives rise to a strong symplectic cobordism from the unit cotangent bundle of the torus to $\left(S^{2 n-1}, \xi_{\text {std }}\right)$, but one can easily find contact forms on the former that have no contractible Reeb orbits, corresponding to metrics on the torus with no contractible geodesics. The cobordism of course cannot be Liouville because, by a well-known theorem of 
Gromov Gro85, the Lagrangian torus cannot be exact. Similarly, Gay06 and Wen13b show that every contact 3-manifold with positive Giroux torsion is strongly symplectically cobordant to something overtwisted, including e.g. the nonfillable tight 3 -tori, which admit contact forms without contractible orbits.

1.3. Applications. Here is a specific situation in which Theorem 1.1 can be used to rule out the existence of exact symplectic cobordisms. Good candidates for manifolds that fail to satisfy the conclusion of the theorem are furnished by the universally tight lens spaces $L(p, q)$ for $p \neq 1$. Recall that $L(p, q)$ is defined as the quotient

$$
L(p, q)=S^{3} / G_{p, q},
$$

where $G_{p, q} \subset \mathrm{U}(2)$ denotes the cyclic group of matrices $\left(\begin{array}{cc}e^{2 \pi i k / p} & 0 \\ 0 & e^{2 \pi i k q / p}\end{array}\right)$ for $k \in \mathbb{Z}_{p}$, acting on the unit sphere $S^{3} \subset \mathbb{C}^{2}$ by unitary transformations. This action preserves the standard contact form $\alpha_{\text {std }}=\frac{1}{2} \sum_{j=1}^{2}\left(x_{j} d y_{j}-\right.$ $\left.y_{j} d x_{j}\right)$ on $S^{3}$, written here in coordinates $\left(z_{1}, z_{2}\right)=\left(x_{1}+i y_{1}, x_{2}+i y_{2}\right)$, so the standard contact structure $\xi_{\text {std }}$ on $L(p, q)$ is defined via this quotient.

Proposition 1.9. For every relatively prime pair of integers $p>q \geq 1$, $L(p, q)$ admits a nondegenerate contact form with only two simple closed Reeb orbits, both of them nondegenerate and noncontractible.

Proof. We present $\left(L(p, q), \xi_{\text {std }}\right)$ as a quotient of the so-called irrational ellipsoid. Let $\alpha_{H}:=\frac{1}{H} \alpha_{\text {std }}$ on $S^{3}$, where $H$ is the restriction to the unit sphere $S^{3} \subset \mathbb{C}^{2}$ of the function

$$
H\left(z_{1}, z_{2}\right)=\frac{\left|z_{1}\right|^{2}}{a^{2}}+\frac{\left|z_{2}\right|^{2}}{b^{2}}
$$

for some $a, b>0$. The closed orbits for the Reeb flow on $S^{3}$ determined by $\alpha_{H}$ are then in bijective correspondence with the closed orbits on the ellipsoid $H^{-1}(1) \subset \mathbb{C}^{2}$ for the Hamiltonian flow of $H$ on the standard symplectic $\mathbb{C}^{2}$. In particular, if $a / b$ is irrational, then the only simple closed orbits of this flow are (up to parametrization) the embedded loops $\gamma_{1}, \gamma_{2}: S^{1} \rightarrow S^{3} \subset \mathbb{C}^{2}$ defined by

$$
\gamma_{1}(t)=\left(e^{2 \pi i t}, 0\right), \quad \gamma_{2}(t)=\left(0, e^{2 \pi i t}\right)
$$

for $t \in S^{1}=\mathbb{R} / \mathbb{Z}$, and moreover, these orbits and their multiple covers are all nondegenerate. Now since $\alpha_{\text {std }}$ and $H$ are both invariant under the action of $\mathrm{U}(1) \times \mathrm{U}(1) \subset \mathrm{U}(2)$, which contains $G_{p, q}, \alpha_{H}$ descends to a welldefined contact form on $L(p, q)$, and this contact form is nondegenerate. But the orbits $\gamma_{1}$ and $\gamma_{2}$ project to orbits in $L(p, q)$ that are $p$-fold covered, so their underlying simple orbits lift to the universal cover $S^{3} \rightarrow L(p, q)$ as non-closed paths since $p>1$, hence they are noncontractible.

Corollary 1.10. For every pair of relatively prime integers $p>q \geq 1$, $\left(L(p, q), \xi_{\text {std }}\right)$ admits no exact cobordism to $\left(S^{3}, \xi_{\text {std }}\right)$.

Remark 1.11. The Reeb flow on any universally tight $L(p, q)$ admits a contractible Reeb orbit since $\pi_{1}(L(p, q))$ is torsion, so previously known criteria for excluding such cobordisms do not apply. 
While the lens space example is relatively easy to work with, the nondegeneracy of a contact form is usually a rather difficult condition to check, and for this reason one might sometimes want to have the following technical enhancement of Theorems 1.1 and 1.2. It will be an immediate consequence of our proofs, requiring only that one pay closer attention to the relationship between periods of orbits and energies of holomorphic curves.

Theorem 1.12. Assume $(M, \xi)$ satisfies the hypotheses of either Theorem 1.1 or Theorem 1.2. and fix a contact form $\alpha_{0}$ for $(M, \xi)$. There exists a constant $T>0$, dependent on $\alpha_{0}$, such that the following holds: suppose $\alpha=f \alpha_{0}$ is a contact form on $(M, \xi)$ such that

(1) $f: M \rightarrow(0, \infty)$ satisfies $f<T$, and

(2) All closed Reeb orbits for $\alpha$ with period less than $T$ are nondegenerate.

Then the Reeb flow of $\alpha$ satisfies the conclusions of Theorems 1.1 or 1.2 respectively, and the unknotted orbit can be assumed to have period less than $T$.

One could apply this in practice if e.g. $\alpha_{0}$ is Morse-Bott and admits no unknotted Reeb orbits, as then one can define perturbations of $\alpha_{0}$ as in Bou02 whose orbits up to some arbitrarily large period are nondegenerate and still knotted - the topology of orbits with large period may be harder to control, but for Theorem 1.12 this does not matter.

Remark 1.13. We have chosen to adopt a mainly contact topological perspective on the main theorems of this paper, but for other purposes (e.g. quantitative Reeb dynamics, cf. GZ13a, §3.23]), one could also state more quantitatively precise versions of Theorem 1.12 .

Note that no such enhancement is necessary for Theorem 1.3, which does not require nondegeneracy, see Remark 1.15.

1.4. Outline of proofs, part 1: seed curves and compactness. All proofs of theorems in this paper follow a similar scheme, which in the case of Theorems 1.1 and 1.3 can be described as follows. Suppose $(W, d \lambda)$ is a Liouville cobordism from $(M, \xi)$ to a standard contact sphere $\left(S^{2 n-1}, \xi_{\text {std }}\right)$, and let $(\bar{W}, d \lambda)$ denote the completion obtained by attaching cylindrical ends in the standard way (see $\$ 2.3$ ). Then the positive end of $\bar{W}$ can be assumed to match the top half of the symplectization

$$
\left(\mathbb{R} \times S^{2 n-1}, d\left(e^{r} \alpha_{\text {std }}\right)\right),
$$

where $\alpha_{\text {std }}$ is the standard contact form, defined by restricting the Liouville form $\lambda_{\text {std }}:=\sum_{j=1}^{n}\left(x_{j} d y_{j}-y_{j} d x_{j}\right)$ to the unit sphere. We will assume also that the negative end matches $\left((-\infty, 0] \times M, d\left(e^{r} \alpha\right)\right)$ where $\alpha$ is (after a positive rescaling) an arbitrary nondegenerate contact form for $(M, \xi)$. (The nondegeneracy assumption was not included in Theorem 1.3, but this assumption will be easy to remove in the final step, see Remark 1.15 below.)

The first step in the proof is then to choose a suitable almost complex structure $J$ on the symplectization (1.1) that admits a foliation by a $(2 n-2)$ dimensional family of $J$-holomorphic planes, so-called "seed curves," which are asymptotic to a fixed Reeb orbit $\gamma$ for $\alpha_{\text {std }}$ that has the smallest possible period. We will be able to verify explicitly that these planes are Fredholm 
regular for the moduli problem with fixed asymptotic orbit, hence the moduli space is cut out transversely, and moreover, there exist no other curves in $\mathbb{R} \times S^{2 n-1}$ with a single positive end approaching $\gamma$. Once these curves are understood, we can regard them as living in the cylindrical end $[0, \infty) \times$ $S^{2 n-1} \subset \bar{W}$, so after extending $J$ to a compatible almost complex structure on the rest of $(\bar{W}, d \lambda)$, they generate a nonempty moduli space $\mathcal{M}(J)$ of unparametrized $J$-holomorphic planes in $\bar{W}$, all asymptotic to the same simply covered Reeb orbit in the sphere, and this moduli space is a smooth $(2 n-2)$-dimensional manifold for generic extensions of $J$ since all curves in $\mathcal{M}(J)$ are somewhere injective. Our main task is then to understand the natural compactification $\overline{\mathcal{M}}(J)$ of $\mathcal{M}(J)$, that is to say, the closure of $\mathcal{M}(J)$ in the space of stable $J$-holomorphic buildings in the sense of $\mathrm{BEH}^{+} 03$. Recall that a $J$-holomorphic building in a cobordism may have multiple levels, including one main level which is a (possibly empty) curve in the completed cobordism, and arbitrary finite numbers of upper levels living in the symplectization of the convex boundary and lower levels living in the symplectization of the concave boundary. The uniqueness of the seed curves in the positive end implies the following:

Lemma 1.14. If $u \in \overline{\mathcal{M}}(J)$ is a stable holomorphic building with a nontrivial upper level, then it has exactly one upper level, which consists of one of the seed curves in $\mathbb{R} \times S^{2 n-1}$, and all its other levels are empty.

The lemma means that the only way for a sequence of planes in $\mathcal{M}(J)$ to "degenerate" with something nontrivial happening at the positive end is if the planes simply escape into the positive end and become seed curves; in particular, this cannot happen to any sequence of planes that have points falling into the negative end. Theorem 1.3 can now be proved as follows. Let $\mathcal{M}_{1}(J)$ denote the smooth $2 n$-dimensional moduli space consisting of curves in $\mathcal{M}(J)$ with the additional data of a marked point, hence there is a well-defined evaluation map

$$
\text { ev }: \mathcal{M}_{1}(J) \rightarrow \bar{W}
$$

Choose a smooth properly embedded 1-dimensional submanifold $\ell \subset \bar{W}$ with one end in $[0, \infty) \times S^{2 n-1}$ and the other in $(-\infty, 0] \times M$, and perturb it to be transverse to the evaluation map. Then

$$
\mathcal{M}_{\ell}(J):=\mathrm{ev}^{-1}(\ell)
$$

is a smooth 1-dimensional manifold, and it has a unique connected component $\mathcal{M}_{\ell}^{0}(J) \subset \mathcal{M}_{\ell}(J)$ that contains seed curves in the positive end. This component has a noncompact end consisting of a family of seed curves that escape to $+\infty$, thus it is manifestly noncompact and therefore diffeomorphic to $\mathbb{R}$. We claim now that $\mathcal{M}_{\ell}^{0}(J)$ must also contain curves with points that descend arbitrarily far into the negative end. Indeed, the SFT compactness theorem would otherwise imply that every sequence in $\mathcal{M}_{\ell}^{0}(J)$ has a subsequence convergent to either an element of $\mathcal{M}_{\ell}^{0}(J)$ or a holomorphic building of the type described in Lemma 1.14. But the latter can only happen if the sequence escapes through the neighbourhood of $+\infty$ in which all curves are seed curves. In particular, we obtain a contradiction by considering a noncompact sequence escaping to the opposite end of $\mathcal{M}_{\ell}^{0}(J) \cong \mathbb{R}$ from the one 

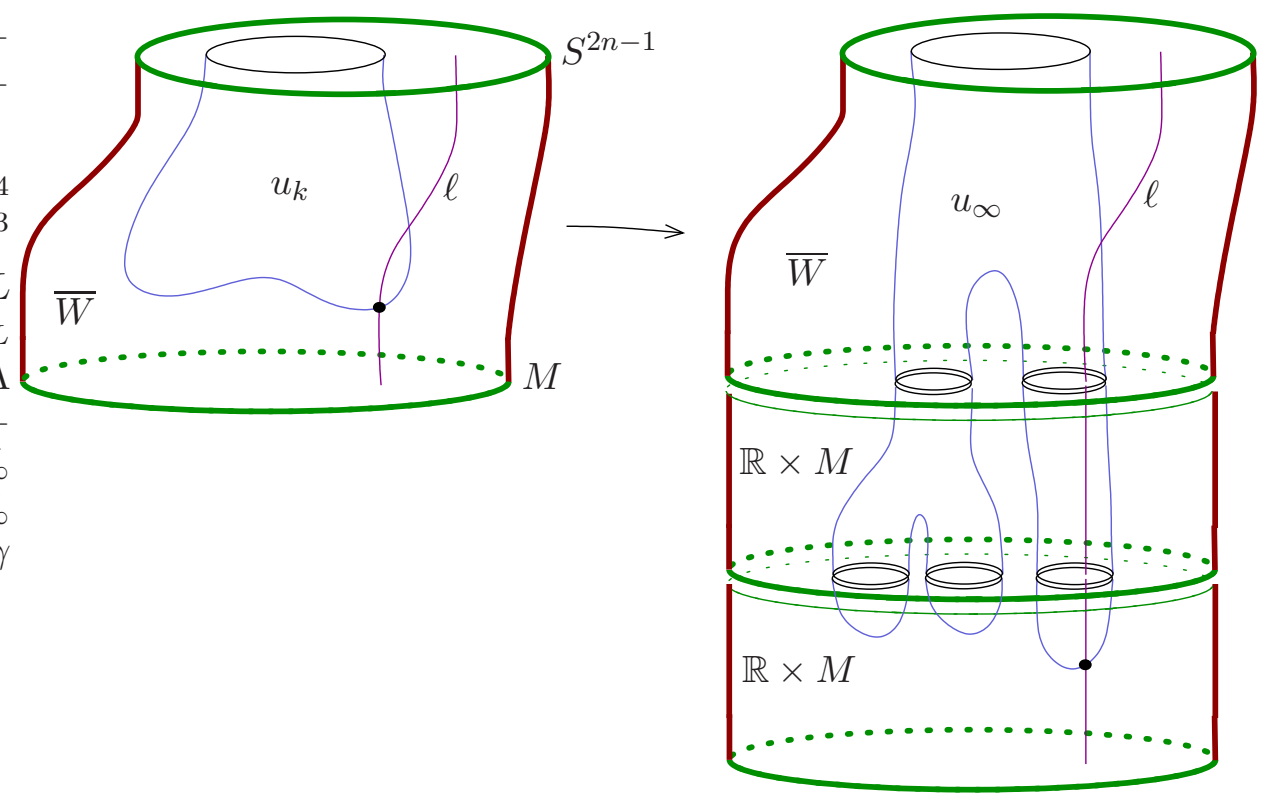

FIgURE 2. When holomorphic planes in an exact cobordism converge to a holomorphic building with nontrivial lower levels, at least one of them must include a plane.

consisting of seed curves, and this proves the claim. It follows that one can find a sequence $u_{k} \in \mathcal{M}_{\ell}(J)$ of curves converging to a holomorphic building $u_{\infty} \in \overline{\mathcal{M}}(J)$ with a nontrivial lower level (see Figure 2). Since the cobordism is exact, every component curve in $u_{\infty}$ must have exactly one positive end, and it follows that at least one of the curves in a lower level of $u_{\infty}$ is a plane, whose asymptotic orbit is the contractible Reeb orbit promised by Theorem 1.3 .

Remark 1.15. To remove the nondegeneracy assumption from Theorem 1.3 , one can take advantage of the fact that due to the exactness of the cobordism, the contractible orbit found in the above argument comes with an a priori bound on its period. Then if $\alpha$ is a degenerate contact form on $(M, \xi)$ approximated by a sequence $\alpha_{k}$ of nondegenerate contact forms, the above argument gives a sequence $\gamma_{k}$ of contractible Reeb orbits with respect to $\alpha_{k}$ whose periods are uniformly bounded, so by Arzelà-Ascoli, these have a subsequence convergent to a contractible Reeb orbit with respect to $\alpha$. Note that if the orbits $\gamma_{k}$ are also unknotted, it is not so clear whether the limiting orbit will also be unknotted, hence the need for the more technical Theorem 1.12 .

1.5. Outline of proofs, part 2: intersections. The argument described thus far is quite standard and, as mentioned earlier, is largely attributable to Hofer [Hof93] (though the use of the path $\ell \subset \bar{W}$ to define a 1-dimensional submanifold of the moduli space is borrowed from Niederkrüger [Nie06]). The arguments required for finding an orbit that is not only contractible but also unknotted are significantly subtler, and here we must make liberal use of Siefring's intersection theory [Sie11] in the low-dimensional setting. 
To explain the idea, we briefly recall the notion of nicely embedded holomorphic curves, introduced by the second author in Wen10a, Wen10b. The precise definition will be reviewed in $\$ 2.4 .5$, but in essence, a holomorphic curve $u: \dot{\Sigma} \rightarrow \bar{W}$ in a completed 4-dimensional symplectic cobordism $\bar{W}$ is nicely embedded if it has the necessary intersection-theoretic properties to guarantee that it does not intersect its neighbors in the moduli space. This condition implies that the moduli space near $u$ can be at most 2 -dimensional, and in the 2-dimensional case the curves near $u$ form the leaves of a foliation on a neighbourhood of $u(\dot{\Sigma})$ in $\bar{W}$. If $\bar{W}$ is a symplectization $\mathbb{R} \times M$ or the image of $u$ is confined to a cylindrical end, then being nicely embedded has the additional implication that $u$ projects to an embedding into $M$, i.e. $u$ can be written as

$$
u=\left(u_{\mathbb{R}}, u_{M}\right): \dot{\Sigma} \rightarrow \mathbb{R} \times M,
$$

where the map $u_{M}: \dot{\Sigma} \rightarrow M$ is also an embedding. It is easy to show that the seed curves we find in the symplectization of $\left(S^{3}, \xi_{\text {std }}\right)$ are nicely embedded, and the homotopy invariance of the intersection theory then implies that the same is true for all curves in $\mathcal{M}(J)$.

The fundamental principle behind the proof of Theorems 1.1 and 1.2 is then the notion that "nice curves degenerate nicely," i.e. if a sequence $u_{k} \in \mathcal{M}(J)$ converges to a holomorphic building $u_{\infty} \in \overline{\mathcal{M}}(J)$, then we should expect the component curves in levels of $u_{\infty}$ to be nicely embedded. This statement as such is false in full generality (see Wen10b, Example 4.22 and Remark 4.23] for counterexamples), but we will show that it is true in the present situation. As a consequence, the plane we find in a lower level of $u_{\infty}$ has the form $\left(u_{\mathbb{R}}, u_{M}\right): \mathbb{C} \rightarrow \mathbb{R} \times M$, where $u_{M}: \mathbb{C} \rightarrow M$ is an embedding asymptotic to a contractible Reeb orbit.

There remains one complication: the fact that $u: \mathbb{C} \rightarrow \mathbb{R} \times M$ is nicely embedded does not guarantee that its asymptotic orbit must be simply covered, i.e. the image of $u_{M}: \mathbb{C} \rightarrow M$ might look like an immersed disk that is embedded on the interior but multiply covered on its boundary. We will show in fact that this can happen, but only in very specific ways, and to prove it, we develop a "local adjunction formula" for holomorphic annuli breaking along a Reeb orbit.

1.6. Local adjunction. We now briefly interrupt the outline of the proof to describe a tool of more general applicability. To set the stage, suppose that $\alpha_{k} \rightarrow \alpha_{\infty}$ is a $\mathcal{C}^{\infty}$-convergent sequence of contact forms on a 3-manifold $M$, and $J_{k} \rightarrow J_{\infty}$ is a corresponding sequence with each $J_{k}$ belonging to the usual space (see 2.1) of admissible translation-invariant almost complex structures on the symplectization $\left(\mathbb{R} \times M, d\left(e^{r} \alpha_{k}\right)\right)$. Assume then that

$$
u_{k}:\left([-k, k] \times S^{1}, i\right) \rightarrow\left(\mathbb{R} \times M, J_{k}\right)
$$

is a sequence of pseudoholomorphic annuli which are converging in the sense of SFT compactness to a broken $J_{\infty}$-holomorphic curve

$$
u_{k} \rightarrow\left(u_{\infty}^{+} \mid u_{\infty}^{-}\right)
$$

where $u_{\infty}^{+}$is the top level with a negative puncture, and $u_{\infty}^{-}$is the bottom level with a positive puncture, both asymptotic to the same nondegenerate Reeb orbit $\gamma$ with covering multiplicity $m(\gamma)$. It is natural to choose 
holomorphic cylindrical coordinates around these punctures and thus parametrize the two levels in the form

$$
\begin{gathered}
u_{\infty}^{+}:\left((-\infty, 0] \times S^{1}, i\right) \rightarrow\left(\mathbb{R} \times M, J_{\infty}\right), \\
u_{\infty}^{-}:\left([0, \infty) \times S^{1}, i\right) \rightarrow\left(\mathbb{R} \times M, J_{\infty}\right),
\end{gathered}
$$

so that the two half-cylinders together can be regarded as a broken holomorphic annulus arising as a limit of the finite (but increasingly long) holomorphic annuli $u_{k}$; see Figure 3. This is intended as a local picture of the neighbourhood of a breaking orbit as a sequence of smooth finite energy curves converges to a holomorphic building as in $\left[\mathrm{BEH}^{+} 03\right]$.

Recall from [Sie08] that for any finite energy punctured holomorphic curve that is not a multiple cover, sufficiently small neighbourhoods of each puncture are always embedded, hence if $u_{\infty}^{+}$and $u_{\infty}^{-}$are not multiply covered then we are free to assume without loss of generality that both are embedded. This implies that each $u_{k}$ is also embedded near the boundary of $[-k, k] \times S^{1}$ for sufficiently large $k$, but if $m(\gamma)>1$, then $u_{k}$ can have finitely many double points and critical points that "disappear into the breaking orbit" in the limit. See 2.4 .5 for precise definitions of each of the quantities discussed below. We let

$$
\delta\left(u_{k}\right) \geq 0
$$

denote the algebraic count of double points and critical points of $u_{k}$ : this is a nonnegative integer that equals zero if and only if $u_{k}$ is embedded. The halfcylinders $u_{\infty}^{ \pm}$are embedded by assumption, but if $m(\gamma)>1$, then they may have "hidden double points at infinity" in the sense of [Sie11, i.e. double points that must emerge from infinity under generic perturbations of the curves. We denote the algebraic counts of these hidden double points by

$$
\delta_{\infty}\left(u_{\infty}^{ \pm}\right) \geq 0
$$

they are nonnegative integers that vanish if and only if generic perturbations of $u_{\infty}^{ \pm}$remain embedded. We denote by

$$
\bar{\sigma}_{ \pm}(\gamma) \geq 1
$$

the so-called spectral covering numbers of $\gamma$ as in [Sie11: these are covering multiplicities of certain asymptotic eigenfunctions of $\gamma$, and are thus positive integers that equal 1 if and only if those eigenfunctions are simply covered (which is always the case e.g. if $m(\gamma)=1$ ). For one last piece of notation, we let

$$
p(\gamma) \in\{0,1\}
$$

denote the parity of $\gamma$, i.e. its Conley-Zehnder index modulo 2. The result we will prove in 44 can now be stated as follows.

Theorem 1.16 (local adjunction). In the setting described above, assume $u_{k} \rightarrow\left(u_{\infty}^{+} \mid u_{\infty}^{-}\right)$is a sequence of holomorphic annuli in $\mathbb{R} \times M$ converging to a broken pair of half-cylinders, where $u_{\infty}^{+}$and $u_{\infty}^{-}$are both embedded and asymptotic to a nondegenerate Reeb orbit $\gamma$ with covering multiplicity $m(\gamma)$, parity $p(\gamma)$ and spectral covering numbers $\bar{\sigma}_{ \pm}(\gamma)$. Then for all $k$ sufficiently 


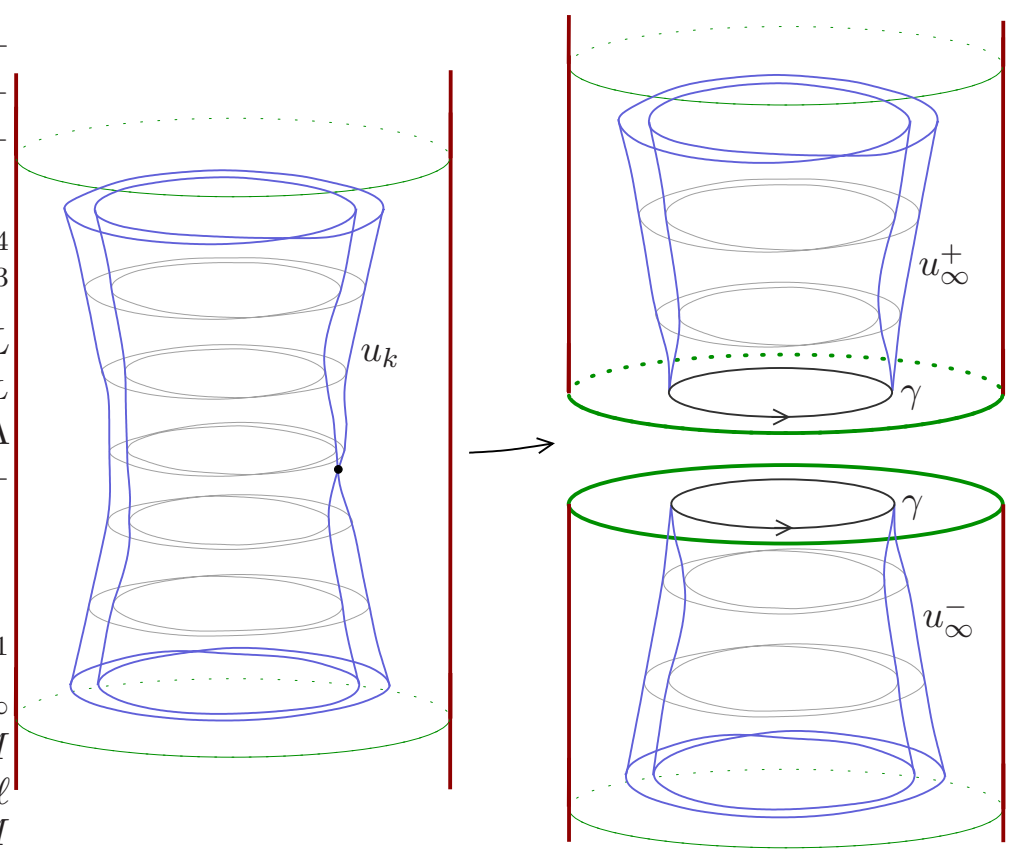

FIgURE 3. A sequence of pseudoholomorphic annuli $u_{k}$ converging to a broken annulus consisting of embedded halfcylinders $u_{\infty}^{ \pm}$asymptotic to a doubly covered breaking orbit $\gamma$. In this case, $u_{k}$ can have double points that disappear in the limit.

large,

$$
\begin{aligned}
2 \delta\left(u_{k}\right)=2\left[\delta_{\infty}\left(u_{\infty}^{+}\right)+\delta_{\infty}\left(u_{\infty}^{-}\right)\right]+\left[\bar{\sigma}_{+}(\gamma)-1\right] & \\
& +\left[\bar{\sigma}_{-}(\gamma)-1\right]+[m(\gamma)-1] p(\gamma) .
\end{aligned}
$$

The usefulness of this theorem lies in the fact that every bracketed term on the right hand side of the formula is known a priori to be nonnegative, so if we also know that the annuli $u_{k}$ are embedded, then all these terms must vanish. In that case, we will easily be able to deduce the following consequence:

Corollary 1.17. In the setting of Theorem 1.16, if $u_{k}$ is embedded for every $k$, then one of the following is true:

- $\gamma$ is a simply covered orbit;

- $\gamma$ is a double cover of a simply covered orbit $\gamma^{\prime}$ such that $p\left(\gamma^{\prime}\right)=1$ and $p(\gamma)=0$, and both of the half-cylinders $u_{\infty}^{ \pm}$have no hidden double points at infinity.

1.7. Outline of proofs, conclusion. In the situation at hand, our degenerating curves are all embedded, so Corollary 1.17 applies and we conclude that the breaking orbit is always either simply covered or a double cover of a negative hyperbolic orbit, what is known in the SFT literature (cf. EGH00]) as a bad orbit. In the first case we are done, and in the second, we will show that degenerations of this form can always be glued back together so that they are interpreted as interior points of the compactified moduli space, 
and the moduli space must therefore have additional degenerations besides this. In other words, breaking along bad orbits can happen, but it cannot be the only type of breaking that happens, so there is still guaranteed to be some breaking along a simple orbit somewhere, producing a nicely embedded curve asymptotic to an unknotted orbit. The resulting constraints on the Conley-Zehnder index and self-linking number of the orbit then follow by a straightforward and essentially standard topological computation.

The major differences between the above summary and the proof of Theorem 1.2 are as follows. For the first statement in the theorem, the symplectic cobordism $W$ is taken to be symplectically trivial, i.e. its completion has the form $(\mathbb{R} \times M, d \lambda)$, where $\lambda$ is a Liouville form matching $e^{r} \alpha_{ \pm}$near $\{ \pm \infty\} \times M$, and $\alpha_{ \pm}$are two nondegenerate contact forms for $(M, \xi)$, of which $\alpha_{-}$is given but $\alpha_{+}$is carefully chosen. The assumptions of the theorem then allow us to choose $\alpha_{+}$and a compatible almost complex structure $J_{+}$near $+\infty$ so that we find a smooth 1-dimensional moduli space of seed curves. Since this moduli space is only 1- and not 2-dimensional, it does not form a foliation, but the curves are still nicely embedded and the same principles therefore apply: a variation on the same argument described above leads to a nicely embedded plane asymptotic to a simple Reeb orbit for $\alpha_{-}$.

Here is an outline of the remainder of the paper. In $\$ 2$, we clarify the essential definitions and review the necessary facts about punctured holomorphic curves and their intersection theory in dimension four. The purpose of 93 is then to specify the data at the positive ends of our symplectic cobordisms, construct the seed curves and prove that they are Fredholm regular and nicely embedded. Theorem 1.16 and Corollary 1.17 on local adjunction for breaking holomorphic annuli are proved in 4 . Finally, \$5 carries out the main compactness arguments, and 96 completes the proofs of the main theorems.

1.8. Acknowledgements. The second author would like to thank Paolo Ghiggini for enlightening discussions, and Emmy Murphy for explaining Example 1.6 and pointing out the connection between exact Lagrangian caps and Liouville cobordisms. The present paper constitutes a portion of the first author's PhD thesis within the framework of the project Intersections in Low-Dimensional Symplectic Field Theory, funded by the Leverhulme Trust. The work of the second author was also partially funded by a Royal Society University Research Fellowship and by EPSRC grant EP/K011588/1.

\section{Preparation}

The purpose of this section is to fix definitions and review some known results that will be needed in the rest of the paper.

2.1. Contact manifolds and symplectic cobordisms. We begin by reviewing some basic definitions from contact geometry and the precise way in which contact manifolds arise as hypersurfaces or boundary components of symplectic manifolds.

Suppose $(W, \omega)$ is a $2 n$-dimensional symplectic manifold, and $M \subset W$ is a smooth oriented hypersurface. We say that $M$ is convex if there exists a Liouville vector field near $M$ that is positively transverse to $M$ : here a 
vector field $V$ is called Liouville if its flow dilates the symplectic form, meaning $\mathcal{L}_{V} \omega=\omega$. This is equivalent to the condition that the dual 1-form $\lambda:=\omega(V, \cdot)$ satisfies $d \lambda=\omega$, and being positively transverse to $M$ then means that the restriction $\alpha:=\left.\lambda\right|_{T M}$ satisfies

$$
\alpha \wedge(d \alpha)^{n-1}>0 \text {. }
$$

This makes $\alpha$ a (positive) contact form on $M$, and the induced (positive and co-oriented) contact structure is the co-oriented hyperplane field $\xi:=$ ker $\alpha \subset T M$. It follows from Gray's stability theorem that if $V$ is replaced with any other Liouville vector field positively transverse to $M$, then the induced contact structure is isotopic to $\xi$, hence the contact form can be regarded as an auxiliary choice, but the contact structure is canonical up to isotopy.

Remark 2.1. In this paper, every contact structure is assumed to be cooriented and positive (with respect to a given orientation of the manifold), and contact forms are always assumed compatible with the given co-orientation.

Example 2.2. We denote by $\xi_{\text {std }} \subset T S^{2 n-1}$ the standard contact structure on the sphere, which arises as the convex boundary of the standard symplectic unit ball with a Liouville vector field pointing radially outward. In coordinates $\left(x_{1}, y_{1}, \ldots, x_{n}, y_{n}\right) \in \mathbb{R}^{2 n}$, the standard contact form $\alpha_{\text {std }}$ is the restriction to $S^{2 n-1} \subset \mathbb{R}^{2 n}$ of the Liouville form $\frac{1}{2} \sum_{j=1}^{n}\left(x_{j} d y_{j}-\right.$ $\left.y_{j} d x_{j}\right)$.

Any choice of contact form $\alpha$ determines a Reeb vector field $R_{\alpha}$ on $M$ via the conditions

$$
d \alpha\left(R_{\alpha}, \cdot\right) \equiv 0, \quad \alpha\left(R_{\alpha}\right) \equiv 1 .
$$

If $M$ is a convex hypersurface in a symplectic manifold $(W, \omega)$, then the orbits of $R_{\alpha}$ are precisely the orbits on $M$ of any Hamiltonian vector field defined by a Hamiltonian function on $(W, \omega)$ with $M$ as a regular level set; moreover, convexity implies that a neighbourhood of $M$ is foliated by other convex hypersurfaces that have the same Reeb orbits up to parametrization. See Gei08 for more on contact structures, and HZ94 for more on the convexity condition in Hamiltonian dynamics.

Given two closed contact manifolds $\left(M_{-}, \xi_{-}\right)$and $\left(M_{+}, \xi_{+}\right)$, a strong symplectic cobordism from $\left(M_{-}, \xi_{-}\right)$to $\left(M_{+}, \xi_{+}\right)$is a compact symplectic manifold $(W, \omega)$ whose boundary can be identified with $-M_{-} \sqcup M_{+}$such that $M_{-}$and $M_{+}$are both convex hypersurfaces and the contact structures they inherit are isotopic to $\xi_{-}$and $\xi_{+}$respectively. Note that the orientation reversal for $M_{-}$means that the Liouville vector field points inward at $M_{-}$ (for this reason we sometimes call $M_{-}$the concave boundary component), whereas it points outward at $M_{+}$. Additionally, $(W, \omega)$ is called a Liouville (or exact symplectic) cobordism from $\left(M_{-}, \xi_{-}\right)$to $\left(M_{+}, \xi_{+}\right)$if the transverse Liouville vector field defined near $\partial W$ can be assumed to extend to a global Liouville vector field. This is equivalent to requiring $\omega=d \lambda$ for some 1-form $\lambda$ that restricts to the boundary as contact forms $\alpha_{ \pm}:=\left.\lambda\right|_{T M_{ \pm}}$ for $\xi_{ \pm}$. 
The symplectization of a contact manifold $(M, \xi=\operatorname{ker} \alpha)$ is the open symplectic manifold $\left(\mathbb{R} \times M, d\left(e^{r} \alpha\right)\right.$ ), where $r$ denotes the coordinate on $\mathbb{R}$. Its symplectic structure is independent of the choice of $\alpha$ up to isotopy, but $\alpha$ determines a special class of compatible almost complex structures $\mathcal{J}(\alpha)$ on $\left(\mathbb{R} \times M, d\left(e^{r} \alpha\right)\right)$ such that $J \in \mathcal{J}(\alpha)$ if and only if:

- $J$ is $\mathbb{R}$-invariant (i.e. invariant under the flow of $\partial_{r}$ );

- $J \partial_{r}=R_{\alpha}$;

- $J(\xi)=\xi$

- $\left.d \alpha(\cdot, J \cdot)\right|_{\xi}$ is a bundle metric on $\xi$.

Given a symplectic cobordism $(W, \omega)$ from $\left(M_{-}, \xi_{-}\right)$to $\left(M_{+}, \xi_{+}\right)$with induced contact forms $\alpha_{ \pm}$at $M_{ \pm}$, the corresponding Liouville vector fields defined near $M_{+}$and $M_{-}$determine collar neighbourhoods $(-\epsilon, 0] \times M_{+}$ and $[0, \epsilon) \times M_{-}$respectively in which $\omega=d\left(e^{r} \alpha_{ \pm}\right)$. One then defines the symplectic completion

$$
\bar{W}=\left((-\infty, 0] \times M_{-}\right) \cup_{M_{-}} W \cup_{M_{+}}\left([0, \infty) \times M_{+}\right)
$$

by extending $\omega$ over the cylindrical ends as $d\left(e^{r} \alpha_{ \pm}\right)$. We shall denote by

$$
\mathcal{J}\left(W, \omega, \alpha_{+}, \alpha_{-}\right)
$$

the (nonempty and contractible) space of almost complex structures on $\bar{W}$ that are $\omega$-compatible on $W$ and restrict to the cylindrical ends as elements of $\mathcal{J}\left(\alpha_{ \pm}\right)$. Almost complex structures of this type will be referred to simply as admissible whenever the corresponding symplectic and contact data is fixed.

2.2. Reeb orbits and the Conley-Zehnder index. Given a contact form $\alpha$ on a contact manifold $(M, \xi)$ of dimension $2 n-1$, a closed Reeb orbit can be regarded as a smooth map

$$
\gamma: S^{1}:=\mathbb{R} / \mathbb{Z} \rightarrow M
$$

satisfying $\dot{\gamma}=T R_{\alpha}(\gamma)$ for some $T>0$, which is the orbit's period. Indeed, setting $x(t):=\gamma(t / T)$, such a map is equivalent to a path $x: \mathbb{R} \rightarrow M$ that satisfies $\dot{x}=R_{\alpha}(x)$ and $x(t+T)=x(t)$ for all $t$. The number $T$ need not generally be the minimal period, hence $\gamma$ may be a multiple cover $\gamma(t)=\gamma_{0}(k t)$ of another closed Reeb orbit $\gamma_{0}$ for some integer $k \geq 2$; when this is not the case, we say $\gamma$ is simple, and the map $\gamma: S^{1} \rightarrow M$ is then an embedding. When $\gamma$ is simple and $\operatorname{dim} M=3$, it makes sense to ask whether $\gamma$ is unknotted, meaning it is the boundary of an embedded disk, or more explicitly there exists an embedding

$$
u: \mathbb{D}^{2} \hookrightarrow M
$$

whose restriction to the boundary coincides with the Reeb orbit:

$$
\left.u\right|_{\partial \mathbb{D}^{2}}=\gamma .
$$

To every closed Reeb orbit one can associate an integer-valued invariant, the Conley-Zehnder index, which depends on a trivialization of the contact structure along the orbit. We will recall the definition of this invariant by way of a theorem regarding asymptotic operators. 
Fix $J \in \mathcal{J}(\alpha)$ and suppose $\gamma: S^{1} \rightarrow M$ is a closed orbit of $R_{\alpha}$ with period $T$. Given any symmetric connection $\nabla$ on $M$, define $A_{\gamma}: \mathcal{C}^{\infty}\left(\gamma^{*} \xi\right) \rightarrow$ $\mathcal{C}^{\infty}\left(\gamma^{*} \xi\right)$ by

$$
A_{\gamma} \eta=-J\left(\nabla_{t} \eta-T \nabla_{\eta} R_{\alpha}\right) .
$$

This operator is well defined and independent of the choice of connection $\nabla$ (see e.g. [Wene, §3.3]), and it is symmetric with respect to the inner product on $\mathcal{C}^{\infty}\left(\gamma^{*} \xi\right)$ defined by

$$
\langle\eta, \zeta\rangle=\int_{S^{1}} \omega_{\gamma(t)}(\eta(t), J(\gamma(t)) \zeta(t)) d t .
$$

It also extends to an unbounded self-adjoint operator on $L^{2}\left(\gamma^{*} \xi\right)$ with domain $W^{1,2}\left(\gamma^{*} \xi\right)$, referred to as the asymptotic operator associated to $\gamma$. Its spectral properties have been described in [HWZ95.

Proposition 2.3 ([HWZ95]). With the notation above, let $\sigma\left(A_{\gamma}\right) \subset \mathbb{R}$ denote the spectrum of $A_{\gamma}$, and for any $\lambda \in \sigma\left(A_{\gamma}\right)$, denote the corresponding eigenspace by $E_{\lambda}$. Then:

(1) $0 \in \sigma\left(A_{\gamma}\right)$ if and only if $\gamma$ is degenerate;

(2) $\sigma\left(A_{\gamma}\right)$ is a discrete subset;

(3) For each $\lambda \in \sigma\left(A_{\gamma}\right), 1 \leq \operatorname{dim} E_{\lambda} \leq 2(n-1)$;

(4) All nontrivial eigenfunctions of $A_{\gamma}$ are everywhere nonzero.

If $\operatorname{dim} M=3$, then the last statement implies that one can define winding numbers wind $^{\Phi}(\eta) \in \mathbb{Z}$ of nontrivial eigenfunctions $\eta$ relative to any fixed unitary trivialization $\Phi$ of $\gamma^{*} \xi$. The following statements then also hold:

(5) If $\eta, \zeta \in E_{\lambda}$ are two nontrivial elements of the same eigenspace, then wind $^{\Phi}(\eta)=\operatorname{wind}^{\Phi}(\zeta)$, hence we can sensibly denote both by $\operatorname{wind}^{\Phi}(\lambda)$.

(6) The map $\sigma\left(A_{\gamma}\right) \rightarrow \mathbb{Z}: \lambda \mapsto$ wind $^{\Phi}(\lambda)$ is 2-to-1 (counting multiplicity of eigenvalues) and increasing. Hence if two distinct eigenvalues have the same winding, they are consecutive and their eigenspaces are 1-dimensional.

It follows that one can speak of the largest negative eigenvalue and the smallest positive eigenvalue associated to the asymptotic operator, and when $\operatorname{dim} M=3$, their winding numbers relative to a chosen trivialization $\Phi$ are denoted by

$$
\alpha_{-}^{\Phi}(\gamma), \quad \alpha_{+}^{\Phi}(\gamma) \in \mathbb{Z}
$$

respectively. Proposition 2.3 implies that these two numbers differ by either 0 or 1 if $\gamma$ is nondegenerate, and in this case, the Conley-Zehnder index relative to the trivialization $\Phi$ of $\gamma^{*} \xi$ can be characterized (according to a theorem in [HWZ95]) via the relation

$$
\mu_{C Z}^{\Phi}(\gamma)=\alpha_{-}^{\Phi}(\gamma)+\alpha_{+}^{\Phi}(\gamma) \in \mathbb{Z},
$$

and its parity (which does not depend on $\Phi$ ) by

$$
p(\gamma)=\alpha_{+}^{\Phi}(\gamma)-\alpha_{-}^{\Phi}(\gamma) \in\{0,1\}
$$

As these formulas indicate, $\mu_{C Z}^{\Phi}(\gamma)$ depends only on the asymptotic operator and can thus sensibly be written as

$$
\mu_{C Z}^{\Phi}\left(A_{\gamma}\right)=\mu_{C Z}^{\Phi}(\gamma)
$$


With this in mind, (2.2) can also be used to compute Conley-Zehnder indices in higher dimensions, via the relation

$$
\mu_{C Z}^{\Phi_{1} \oplus \ldots \oplus \Phi_{m}}\left(A_{1} \oplus \ldots \oplus A_{m}\right)=\mu_{C Z}^{\Phi_{1}}\left(A_{1}\right)+\ldots+\mu_{C Z}^{\Phi_{m}}\left(A_{m}\right),
$$

which holds for any collection of asymptotic operators $A_{j}$ with trivial kernels on Hermitian line bundles trivialized by $\Phi_{j}$ for $j=1, \ldots, m$. We will use this to compute the indices of higher-dimensional seed curves in 3.1 .

While $\mu_{C Z}^{\Phi}(\gamma)$ depends generally on the choice of trivialization $\Phi$, in certain situations one can make natural choices to remove this ambiguity. If $\gamma$ is nullhomologous and forms the boundary of an immersed surface $\mathcal{D}$ in $M$, we define

$$
\mu_{\mathrm{CZ}}(\gamma ; \mathcal{D}) \in \mathbb{Z}
$$

as $\mu_{C Z}^{\Phi}(\gamma)$ with $\Phi$ required to admit an extension to a unitary trivialization of $\xi$ along $\mathcal{D}$. The index in this case still depends on the choice of surface $\mathcal{D}$, but this ambiguity also disappears if $c_{1}(\xi)=0$, which is true e.g. on $\left(S^{3}, \xi_{\text {std }}\right)$.

We require the following standard lemma on the behaviour of the index for multiply covered orbits in dimension three. Let

$$
\gamma^{k}: S^{1} \rightarrow M: t \mapsto \gamma(k t)
$$

denote the $k$-fold cover of the orbit $\gamma: S^{1} \rightarrow M$ for $k \in \mathbb{N}$, and note that any trivialization $\Phi$ of $\gamma^{*} \xi$ induces a trivialization $\Phi^{k}$ of $\left(\gamma^{k}\right)^{*} \xi$.

Lemma 2.4. Suppose $\operatorname{dim} M=3$, and that $\gamma$ and all its multiple covers are nondegenerate. Then for any unitary trivialization $\Phi$ of $\gamma^{*} \xi$,

$$
\mu_{C Z}^{\Phi^{k}}\left(\gamma^{k}\right)= \begin{cases}k \cdot \mu_{C Z}^{\Phi}(\gamma) & \text { if } \gamma \text { is hyperbolic } \\ 2\lfloor k \theta\rfloor+1 & \text { if } \gamma \text { is elliptic }\end{cases}
$$

for every $k \in \mathbb{N}$, where in the elliptic case, $\theta \in \mathbb{R}$ is an irrational number determined by $\gamma$ and $\Phi$.

We will occasionally also need to deal with Reeb orbits $\gamma$ that are degenerate but belong to Morse-Bott families, in which case the following definition will be convenient. If $\gamma$ is degenerate, then $0 \in \sigma\left(A_{\gamma}\right)$ but one can find $\epsilon>0$ such that $(-\epsilon, 0) \cap \sigma\left(A_{\gamma}\right)=\emptyset$. It follows that for any $\epsilon>0$ sufficiently small, $A_{\gamma}+\epsilon$ is the asymptotic operator of a perturbed nondegenerate orbit, whose index we will denote by

$$
\mu_{C Z}^{\Phi}(\gamma+\epsilon):=\mu_{C Z}^{\Phi}\left(A_{\gamma}+\epsilon\right) .
$$

This is independent of the choice as long as $\epsilon>0$ is sufficiently small, and this perturbed Conley-Zehnder index gives a sharp lower bound on the indices of possible nondegenerate perturbations of $\gamma$. The winding numbers $\alpha_{ \pm}^{\Phi}(\gamma+\epsilon) \in \mathbb{Z}$ are defined similarly after replacing $A_{\gamma}$ by $A_{\gamma}+\epsilon$, and they are then related to $\mu_{C Z}^{\Phi}(\gamma+\epsilon)$ by the obvious analogue of (2.2). Notice that $\alpha_{-}^{\Phi}(\gamma+\epsilon)=\alpha_{-}^{\Phi}(\gamma)$, but $\alpha_{+}^{\Phi}(\gamma+\epsilon)$ and $\alpha_{+}^{\Phi}(\gamma)$ may differ if $\gamma$ is degenerate.

Finally, here is a definition that will be needed for intersection theory when $\operatorname{dim} M=3$. Observe that for any Reeb orbit $\gamma_{0}$ and integers $k \geq 2$, every eigenfunction in the $\lambda$-eigenspace of $A_{\gamma_{0}}$ has a $k$-fold cover that belongs to the $k \lambda$-eigenspace of $A_{\gamma_{0}^{k}}$. In the three-dimensional case, one can use Proposition 2.3 to show that the covering multiplicity of an eigenfunction depends only on its winding number, thus all elements of the same eigenspace 
have the same covering multiplicity. The (positive and negative) spectral covering numbers

$$
\bar{\sigma}_{ \pm}(\gamma) \in \mathbb{N}
$$

are defined as the covering multiplicity of the eigenspace that has winding $\alpha_{ \pm}^{\Phi}(\gamma)$. Note that this is only interesting when $\gamma=\gamma_{0}^{k}$ for some other orbit $\gamma_{0}$ and $k \geq 2$; if $\gamma$ is simple then $\bar{\sigma}_{ \pm}(\gamma)=1$ always.

2.3. Holomorphic curves in completed symplectic cobordisms. In this subsection, fix a $2 n$-dimensional symplectic cobordism $(W, \omega)$ with completion $\bar{W}$ and admissible almost complex structure $J \in \mathcal{J}\left(W, \omega, \alpha_{+}, \alpha_{-}\right)$, with the restrictions of $J$ to the cylindrical ends denoted by $J_{ \pm} \in \mathcal{J}\left(\alpha_{ \pm}\right)$.

2.3.1. Asymptotics. We will consider asymptotically cylindrical pseudoholomorphic curves $u:(\dot{\Sigma}, j) \rightarrow(\bar{W}, J)$, where

$$
\dot{\Sigma}=\Sigma \backslash \Gamma
$$

is the result of removing finitely many punctures $\Gamma \subset \Sigma$ from a closed Riemann surface $(\Sigma, j)$. The set of punctures is partitioned into sets of positive and negative punctures $\Gamma^{+}$and $\Gamma^{-}$respectively, where $z \in \Gamma^{ \pm}$means that one can find a biholomorphic identification of a punctured neighbourhood of $z$ with $[0, \infty) \times S^{1}$ or $(-\infty, 0] \times S^{1}$ respectively such that for $|s|$ sufficiently large, $u$ in these coordinates takes the form

$$
u(s, t)=\exp _{(T s, \gamma(t))} h(s, t) \in[0, \infty) \times M_{+} \text {or }(-\infty, 0] \times M_{-}
$$

for some closed Reeb orbit $\gamma: S^{1} \rightarrow M_{ \pm}$with period $T>0$, where the exponential map is defined with respect to any choice of translation-invariant metric on the cylindrical ends, and $h(s, t)$ is a vector field along the trivial cylinder which satisfies $|h(s, t)| \rightarrow 0$ as $s \rightarrow \pm \infty$. We say in this case that $u$ is (positively or negatively) asymptotic to $\gamma$ at $z$, and $h(s, t)$ is called the asymptotic representative of $u$ at $z$. The asymptotic behaviour of $h(s, t)$ is described by a formula proved in [HWZ96a, HWZ96b, Mor03, Sie08]: namely if the orbit $\gamma$ is nondegenerate or Morse-Bott, then for $|s|$ sufficiently large, $h$ is either identically zero or satisfies

$$
h(s, t)=e^{\lambda s}\left(e_{1}(t)+r(s, t)\right),
$$

where $r(s, t) \rightarrow 0$ uniformly in all derivatives as $s \rightarrow \pm \infty, \lambda \in \sigma\left(A_{\gamma}\right)$ is an eigenvalue of the asymptotic operator of $\gamma$ with $\pm \lambda<0$, and $e_{1} \in \mathcal{C}^{\infty}\left(\gamma^{*} \xi_{ \pm}\right)$ is a nontrivial element of the corresponding eigenspace.

2.3.2. Moduli spaces and compactness. It is a standard fact that every asymptotically cylindrical $J$-holomorphic curve $u:(\dot{\Sigma}, j) \rightarrow(\bar{W}, J)$ either is somewhere injective or is a multiple cover of a somewhere injective asymptotically cylindrical curve, and moreover, the set of injective points of a somewhere injective curve is open and dense. A complete proof of this statement may be found in [Nel15], using asymptotic results of Siefring [Sie08]. Recall that $z \in \dot{\Sigma}$ is called an injective point of $u$ if $u^{-1}(u(z))=\{z\}$ and $d u(z) \neq 0$, and we call $u$ a $k$-fold multiple cover of another curve $v:\left(\dot{\Sigma}^{\prime}=\Sigma^{\prime} \backslash \Gamma^{\prime}, j^{\prime}\right) \rightarrow(\bar{W}, J)$ if

$$
u=v \circ \phi
$$


for some holomorphic map $\phi:(\Sigma, j) \rightarrow\left(\Sigma^{\prime}, j^{\prime}\right)$ of degree $k$.

Fix finite ordered tuples of Reeb orbits $\gamma^{+}=\left(\gamma_{1}^{+}, \ldots, \gamma_{k_{+}}^{+}\right)$and $\gamma^{-}=$ $\left(\gamma_{1}^{-}, \ldots, \gamma_{k_{-}}^{-}\right)$in $M_{+}$and $M_{-}$respectively (the case $k_{ \pm}=0$ is allowed), assuming that all of them are either nondegenerate or belong to Morse-Bott families. For an integer $m \geq 0$, the moduli space

$$
\mathcal{M}_{m}\left(J, \gamma^{+}, \gamma^{-}\right)
$$

of unparametrized $J$-holomorphic spheres asymptotic to $\gamma^{+}$and $\gamma^{-}$with $m$ marked points is defined as the set of equivalence classes of tuples $\left(\Sigma, j, \Gamma^{+}, \Gamma^{-}, u,\left(\zeta_{1}, \ldots, \zeta_{m}\right)\right)$ where $(\Sigma, j)$ is a closed Riemann surface of genus zero, $\Gamma^{+}, \Gamma^{-} \subset \Sigma$ are disjoint finite sets, each equipped with an ordering, the marked points $\zeta_{1}, \ldots, \zeta_{m} \in \dot{\Sigma}:=\Sigma \backslash\left(\Gamma^{+} \cup \Gamma^{-}\right)$are all distinct, and

$$
u:(\dot{\Sigma}, j) \rightarrow(\bar{W}, J)
$$

is an asymptotically cylindrical $J$-holomorphic curve with positive punctures $\Gamma^{+}$and negative punctures $\Gamma^{-}$, such that $u$ is asymptotic at the $i$ th puncture in $\Gamma^{ \pm}$to $\gamma_{i}^{ \pm}$for $i=1, \ldots, k_{ \pm}$. Two such tuples are considered equivalent if one can be written as a reparametrization of the other via a biholomorphic diffeomorphism of their domains that maps marked points to marked points and punctures to punctures, with signs and orderings preserved. The topology of $\mathcal{M}_{m}\left(J, \gamma^{+}, \gamma^{-}\right)$can be characterized by saying that a sequence converges if it has representatives with a fixed domain $\Sigma$ and fixed sets of punctures and marked points such that the conformal structures converge in $\mathcal{C}^{\infty}(\Sigma)$ while the maps to $\bar{W}$ converge in $\mathcal{C}_{\text {loc }}^{\infty}(\dot{\Sigma})$ and also in $\mathcal{C}^{0}$ up to infinity (with respect to translation-invariant metrics on the cylindrical ends). We shall often abuse notation by referring to the entire equivalence class of tuples $\left[\left(\Sigma, j, \Gamma^{+}, \Gamma^{-}, u,\left(\zeta_{1}, \ldots, \zeta_{m}\right)\right)\right]$ forming an element of $\mathcal{M}_{m}\left(J, \gamma^{+}, \gamma^{-}\right)$ simply as $u$. In this paper we will only consider the cases $m=0,1$, abbreviating the former by

$$
\mathcal{M}\left(J, \gamma^{+}, \gamma^{-}\right):=\mathcal{M}_{0}\left(J, \gamma^{+}, \gamma^{-}\right) .
$$

For $m>0$, the evaluation map

$$
\begin{aligned}
\mathrm{ev}: \mathcal{M}_{m}\left(J, \gamma^{+}, \gamma^{-}\right) & \rightarrow \bar{W}^{m} \\
{\left[\left(\Sigma, j, \Gamma^{+}, \Gamma^{-}, u,\left(\zeta_{1}, \ldots, \zeta_{m}\right)\right)\right] } & \mapsto\left(u\left(\zeta_{1}\right), \ldots, u\left(\zeta_{m}\right)\right)
\end{aligned}
$$

is well defined and continuous by construction.

Recall that neighbourhoods in $\mathcal{M}_{m}\left(J, \gamma^{+}, \gamma^{-}\right)$can be described as zerosets of smooth Fredholm sections in suitable Banach space bundles (see e.g. Wen10b]). A curve $u$ is called Fredholm regular whenever it forms a transverse intersection of such a Fredholm section with the zero-section. The virtual dimension of $\mathcal{M}_{m}\left(J, \gamma^{+}, \gamma^{-}\right)$at $u$ is given by the Fredholm index of the linearized section at $u$ minus the dimension of the group of automorphisms of the domain, and in the case $m=0$ is also called the index of $u$. If the orbits are all nondegenerate, it is given by the formula

$$
\operatorname{ind}(u)=(n-3) \chi(\dot{\Sigma})+2 c_{1}^{\Phi}\left(u^{*} T \bar{W}\right)+\sum_{i=1}^{k_{+}} \mu_{C Z}^{\Phi}\left(\gamma_{i}^{+}\right)-\sum_{i=1}^{k_{-}} \mu_{C Z}^{\Phi}\left(\gamma_{i}^{-}\right) .
$$


Here $\Phi$ is an arbitrary choice of unitary trivializations of $\xi_{ \pm}$along each of the asymptotic orbits, which naturally induce asymptotic trivializations of the complex vector bundle $u^{*} T \bar{W} \rightarrow \dot{\Sigma}$, and $c_{1}^{\Phi}\left(u^{*} T \bar{W}\right) \in \mathbb{Z}$ then denotes the relative first Chern number of $u^{*} T \bar{W}$ with respect to these asymptotic trivializations. This term ensures that the total expression is independent of the choice $\Phi$. We will also need a special case of the index formula under Morse-Bott assumptions: if all positive asymptotic orbits are Morse-Bott (but possibly degenerate) and all negative orbits are nondegenerate, then

$$
\operatorname{ind}(u)=(n-3) \chi(\dot{\Sigma})+2 c_{1}^{\Phi}\left(u^{*} T \bar{W}\right)+\sum_{i=1}^{k_{+}} \mu_{C Z}^{\Phi}\left(\gamma_{i}^{+}+\epsilon\right)-\sum_{i=1}^{k_{-}} \mu_{C Z}^{\prime}\left(\gamma_{i}^{-}\right)
$$

where $\epsilon>0$ is assumed sufficiently small (see (2.6)). Note that this is the virtual dimension of the moduli space of curves near $u$ with fixed asymptotic orbits, i.e. the orbits are not allowed to move continuously in their respective Morse-Bott families. The index without this constraint would be larger; see Wen10b for an explanation of (2.9) and the constrained/unconstrained distinction. Adding a marked point generally increases the virtual dimension by 2 , so $\mathcal{M}_{m}\left(J, \boldsymbol{\gamma}^{+}, \boldsymbol{\gamma}^{-}\right)$has virtual dimension ind $(u)+2 m$ on any component that includes the curve $u \in \mathcal{M}\left(J, \gamma^{+}, \gamma^{-}\right)$.

A standard application of the implicit function theorem implies that the open subset consisting of Fredholm regular curves in $\mathcal{M}_{m}\left(J, \gamma^{+}, \gamma^{-}\right)$admits the structure of a smooth finite-dimensional orbifold whose dimension locally equals its virtual dimension, and it is a manifold near any curve that is somewhere injective. Moreover, a standard argument via the Sard-Smale theorem (see [MS04 or [Wenc]) shows that after perturbing $J$ generically in $\mathcal{J}\left(W, \omega, \alpha_{+}, \alpha_{-}\right)$on some open subset $\mathcal{U} \subset W$ with compact closure, one can assume that all somewhere injective curves passing through $\mathcal{U}$ are Fredholm regular. Similarly, Dragnev [Dra04] (see also Wenb]) has shown that on a symplectization $\left(\mathbb{R} \times M, d\left(e^{r} \alpha\right)\right)$, generic perturbations within $\mathcal{J}\left(\alpha_{ \pm}\right)$suffice to make all somewhere injective curves regular, and this result can also be applied to any curves in the cobordism $\bar{W}$ that are contained in a cylindrical end.

If the Reeb flows on $M_{+}$and $M_{-}$are both globally nondegenerate or Morse-Bott, then $\mathcal{M}_{m}\left(J, \gamma^{+}, \gamma^{-}\right)$has a natural compactification

$$
\overline{\mathcal{M}}_{m}\left(J, \gamma^{+}, \gamma^{-}\right)
$$

defined in $\mathrm{BEH}^{+} 03$, consisting of stable holomorphic buildings of arithmetic genus zero with $m$ marked points. An example of a holomorphic building (with higher arithmetic genus) is shown in Figure 4. We shall write holomorphic buildings using the notation

$$
\left(v_{N_{+}}^{+}|\ldots| v_{1}^{+}\left|v_{0}\right| v_{1}^{-}|\ldots| v_{N_{-}}^{-}\right)
$$

where $N_{+}, N_{-} \geq 0$ are integers, $v_{1}^{ \pm}, \ldots, v_{N_{ \pm}}^{ \pm}$are each (possibly disconnected and/or nodal) $J_{ \pm}$-holomorphic curves in the symplectizations $\mathbb{R} \times M_{ \pm}$, forming the upper and lower levels respectively, and $v_{0}$ is a (possibly disconnected and/or nodal) $J$-holomorphic curve in $\bar{W}$, the main level. Note that by convention, the main level is allowed to be empty (i.e. $v_{0}$ is a curve with domain the empty set) if $N_{+}$or $N_{-}$is nonzero. Each upper or level 
is defined only up to $\mathbb{R}$-translation, and the same is true of all levels when $\bar{W}$ is a symplectization, in which case there is no distinguished "main" level or distinction between "upper" and "lower" levels. The evaluation map extends continuously over $\overline{\mathcal{M}}_{m}\left(J, \gamma^{+}, \gamma^{-}\right)$if we also compactify $\bar{W}$ by adding $\{ \pm \infty\} \times M_{ \pm}$to the top and bottom of the cylindrical ends, i.e. marked points in upper or lower levels are mapped to $\{\infty\} \times M_{+}$or $\{-\infty\} \times M_{-}$ respectively.

Our notation for buildings is convenient but suppresses an additional detail that will sometimes be quite important: the data also includes a oneto-one corresondence between the positive punctures of each level (other than the topmost) and the negative punctures of the level above it, such that corresponding punctures have matching asymptotic orbits, the so-called breaking orbits. Additionally, each pair of corresponding punctures is equipped with a choice of a rotation angle for gluing the corresponding positive and negative ends along the breaking orbit - this choice is unique if the orbit is simple, but in general there are $m \in \mathbb{N}$ distinct choices if the orbit has covering multiplicity $m$. All of this data together is called a decoration of the building. Different choices of decoration often produce buildings that are biholomorphically inequivalent to each other and thus represent distinct elements of $\overline{\mathcal{M}}_{m}\left(J, \gamma^{+}, \gamma^{-}\right)$.

Whenever $(W, \omega)$ is a Liouville cobordism (and in particular if $\bar{W}$ is a symplectization), Stokes' theorem prevents the existence of curves with no positive ends, sometimes referred to as holomorphic caps. The following standard result is then immediate from the definition of convergence in $\mathrm{BEH}^{+} 03$.

Proposition 2.5. Suppose $\bar{W}$ is either a symplectization or the completion of a Liouville cobordism, and $u_{k} \in \mathcal{M}_{m}(J, \gamma, \emptyset)$ is a sequence of $J$ holomorphic planes converging to a holomorphic building. Then the limiting building has the following properties:

- Each connected component of each level is a punctured sphere with precisely one positive puncture.

- The lowest level has no negative punctures (so it is a disjoint union of planes).

- The top level is connected.

- There are no nodes.

We shall refer to the components without negative ends in the above lemma as capping planes; they are not to be confused with "holomorphic caps," which have only negative ends.

The converse of compactness is gluing, as discussed e.g. in Nel13, Chapter 7]. We will only need the following special case.

Proposition 2.6. Assume $\gamma_{\infty}$ is a Morse-Bott Reeb orbit in $M_{+}, \gamma$ is a nondegenerate orbit in $M_{-}, m \geq 0$ is an integer, and $\left(v_{0} \mid v_{1}^{-}\right) \in \overline{\mathcal{M}}_{m}\left(J, \gamma_{\infty}, \emptyset\right)$ is a (decorated) stable $J$-holomorphic building such that $v_{0} \in \mathcal{M}_{m}\left(J, \gamma_{\infty}, \gamma\right)$ and $v_{1}^{-} \in \mathcal{M}\left(J_{-}, \gamma, \emptyset\right) / \mathbb{R}$ are both somewhere injective and Fredholm regular. 


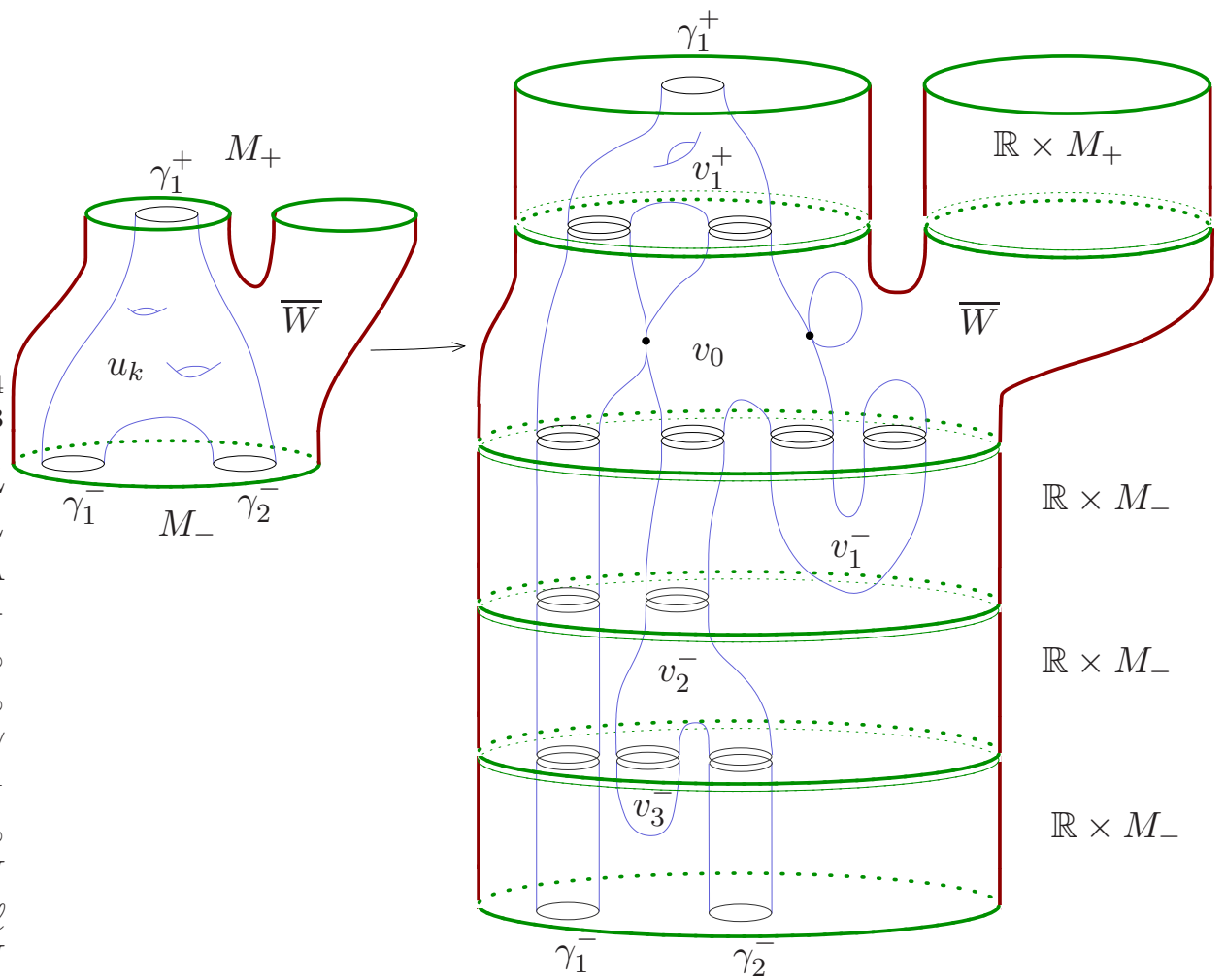

Figure 4. The picture shows the degeneration of a sequence of punctured curves of genus 2 into a building with a main level, one upper level and three lower levels. We label the building as $\left(v_{1}^{+}\left|v_{0}\right| v_{1}^{-}\left|v_{2}^{-}\right| v_{3}^{-}\right)$, where each $v_{i}^{ \pm}$is in general a disconnected nodal curve in a single level. The arithmetic genus of the building is still 2 , and the levels match along their respective asymptotic orbits.

Then there exist neighborhoods

$$
\begin{aligned}
v_{0} & \in \mathcal{U}_{0} \subset \mathcal{M}_{m}\left(J, \gamma_{\infty}, \gamma\right) \\
v_{1}^{-} & \in \mathcal{U}_{-} \subset \mathcal{M}\left(J_{-}, \gamma, \emptyset\right) / \mathbb{R}
\end{aligned}
$$

and a smooth embedding

$$
\Psi:[0, \infty) \times \mathcal{U}_{0} \times \mathcal{U}_{-} \hookrightarrow \mathcal{M}_{m}\left(J, \gamma_{\infty}, \emptyset\right)
$$

such that for any sequences $[0, \infty) \ni r_{k} \rightarrow+\infty, u_{k} \rightarrow u_{\infty} \in \mathcal{U}_{0}$ and $u_{k}^{-} \rightarrow$ $u_{\infty}^{-} \in \mathcal{U}_{-}$,

$$
\Psi\left(r_{k}, u_{k}, u_{k}^{-}\right) \rightarrow\left(u_{\infty} \mid u_{\infty}^{-}\right) \in \overline{\mathcal{M}}_{m}\left(J, \gamma_{\infty}, \emptyset\right)
$$

in the SFT topology. Moreover, every smooth curve in $\mathcal{M}_{m}\left(J, \gamma_{\infty}, \emptyset\right)$ sufficiently close to $\left(v_{0} \mid v_{1}^{-}\right)$in the SFT topology is in the image of $\Psi$.

Remark 2.7. The notation for buildings used in Proposition 2.6 implicitly assumes that if multiple buildings can be constructed out of $u_{\infty}$ and $u_{\infty}^{-}$via different choices of decoration, then $\left(u_{\infty} \mid u_{\infty}^{-}\right)$is the unique choice that is close to $\left(v_{0} \mid v_{1}^{-}\right)$in the SFT topology. 
2.4. The low-dimensional case. We now specialize to the case where the cobordism $(W, \omega)$ is 4-dimensional, so all contact manifolds under consideration will be 3 -dimensional.

2.4.1. Indices of covers. We begin with a pair of convenient numerical observations. The first is borrowed (along with its proof) from Hut02.

Proposition 2.8. Suppose $J \in \mathcal{J}(\alpha)$ for a contact 3 -manifold $(M, \xi=$ ker $\alpha)$, and $u:(\dot{\Sigma}, j) \rightarrow(\mathbb{R} \times M, J)$ is a J-holomorphic branched cover of a trivial cylinder over a Reeb orbit whose covers are all nondegenerate. Then $\operatorname{ind}(u) \geq 0$, and equality can hold only when the cover is unbranched or the orbit is elliptic.

Proof. If the underlying orbit $\gamma$ is hyperbolic, then the index formula gives $\operatorname{ind}(u)=-\chi(\dot{\Sigma}) \geq 0$ due to Lemma 2.4 which is an equality if and only if $\dot{\Sigma}$ is the cylinder, in which case the Riemann-Hurwitz formula implies that the cover is unbranched. If the orbit is instead elliptic, we can make our lives slightly easier with the observation that $u$ has the same index as that of some holomorphic building whose connected components are all thricepunctured spheres that are also branched covers of the same trivial cylinder. It therefore suffices to prove that the inequality holds for thrice-punctured spheres. If for instance $u$ has two positive punctures at $\gamma^{k}$ and $\gamma^{\ell}$ and a negative puncture at $\gamma^{k+\ell}$, then Lemma 2.4 gives

$$
\operatorname{ind}(u)=-\chi(\dot{\Sigma})+(2\lfloor k \theta\rfloor+1)+(2\lfloor\ell \theta\rfloor+1)-(2\lfloor(k+\ell) \theta\rfloor+1),
$$

where $\chi(\dot{\Sigma})=-1$, and the index is thus nonnegative due to the relation $\lfloor a+b\rfloor \leq\lfloor a\rfloor+\lfloor b\rfloor+1$. In the inverse case with one positive puncture and two negative, we get the same result using $\lfloor a\rfloor+\lfloor b\rfloor \leq\lfloor a+b\rfloor$.

Proposition 2.9. Suppose $\operatorname{dim} W=4$ and $u=v \circ \phi:(\dot{\Sigma}, j) \rightarrow(\bar{W}, J)$ is a $k$-fold cover of a somewhere injective J-holomorphic curve $v:\left(\dot{\Sigma}^{\prime}, j^{\prime}\right) \rightarrow$ $(\bar{W}, J)$ whose asymptotic orbits are all nondegenerate and hyperbolic. Then

$$
\operatorname{ind}(u) \geq k \operatorname{ind}(v) \text {, }
$$

with equality if and only if the cover $\phi:(\dot{\Sigma}, j) \rightarrow\left(\dot{\Sigma}^{\prime}, j^{\prime}\right)$ has no branch points in the punctured surface $\dot{\Sigma}$.

Proof. This is a direct consequence of the index formula (2.8) together with Lemma 2.4 and the Riemann-Hurwitz formula $Z(d \phi)=-\chi(\dot{\Sigma})+k \chi\left(\dot{\Sigma}^{\prime}\right)$, where $Z(d \phi) \geq 0$ denotes the algebraic count of zeroes of the holomorphic section $d \phi \in \Gamma\left(\operatorname{Hom}_{\mathbb{C}}\left(T \dot{\Sigma}, \phi^{*} T \dot{\Sigma}^{\prime}\right)\right)$, and thus vanishes if and only if the cover is unbranched.

2.4.2. Asymptotic defect. Suppose $u: \dot{\Sigma} \rightarrow \bar{W}$ is asymptotic at $z \in \Gamma^{ \pm}$ to a $T$-periodic orbit $\gamma: S^{1} \rightarrow M_{ \pm}$and has an asymptotic representative $h(s, t)$ at this puncture that is not identically zero. Then the asymptotic formula (2.7) provides a nonzero eigenfunction $e_{1} \in \mathcal{C}^{\infty}\left(\gamma^{*} \xi_{ \pm}\right)$, and given a trivialization $\Phi$ of $\gamma^{*} \xi_{ \pm}$, one can define

$$
\operatorname{wind}_{\infty}^{\Phi}(u ; z):=\operatorname{wind}^{\Phi}\left(e_{1}\right) \in \mathbb{Z} .
$$

If $z \in \Gamma^{+}$, then $\alpha_{-}^{\Phi}(\gamma)$ is the winding of the greatest negative eigenvalue of $A_{\gamma}$, thus $\operatorname{wind}_{\infty}^{\Phi}(u ; z) \leq \alpha_{-}^{\Phi}(\gamma)$, and similarly, $\operatorname{wind}_{\infty}^{\Phi}(u ; z) \geq \alpha_{+}^{\Phi}(\gamma)$ if 
$z \in \Gamma^{-}$. The difference $\alpha_{-}^{\Phi}(\gamma)-\operatorname{wind}_{\infty}^{\Phi}(u ; z)$ or $\operatorname{wind}_{\infty}^{\Phi}(u ; z)-\alpha_{+}^{\Phi}(\gamma)$ for a positive or negative puncture respectively is denoted $d_{0}(u ; z) \geq 0$ and called the asymptotic defect of $u$ at $z \in \Gamma$. Notice that it does not depend on the trivialization. The total asymptotic defect of $u$ is then a nonnegative integer

$$
d_{0}(u)=\sum_{z \in \Gamma} d_{0}(u ; z) .
$$

This is well defined for any curve $u$ that is not identical to a trivial cylinder in some neighbourhood of any of its punctures; in particular, if $\bar{W}$ is a symplectization $\left(\mathbb{R} \times M, d\left(e^{r} \alpha\right)\right)$ with $J \in \mathcal{J}(\alpha)$, then $d_{0}(u)$ is well defined for every curve other than covers of trivial cylinders.

2.4.3. The normal Chern number and $\operatorname{wind}_{\pi}(u)$. The normal Chern number of a curve $u \in \mathcal{M}\left(J, \gamma^{+}, \gamma^{-}\right)$with all asymptotic orbits nondegenerate is defined by

$$
c_{N}(u)=c_{1}^{\Phi}\left(u^{*} T \bar{W}\right)-\chi(\dot{\Sigma})+\sum_{i=1}^{k_{+}} \alpha_{-}^{\Phi}\left(\gamma_{i}^{+}\right)-\sum_{i=1}^{k_{-}} \alpha_{+}^{\Phi}\left(\gamma_{i}^{-}\right),
$$

where $\Phi$ is again an arbitrary choice of unitary trivializations of $\xi_{ \pm}$along the asymptotic orbits, and the sum does not depend on this choice. The index formula and relations between Conley-Zehnder indices and winding numbers imply

$$
2 c_{N}(u)=\operatorname{ind}(u)-2+2 g+\# \Gamma_{0},
$$

where $g \geq 0$ is the genus of the domain (zero in our case) and $\Gamma_{0} \subset \Gamma$ denotes the set of punctures of $u$ that have even parity. In the Morse-Bott setting of (2.9), the definition of $c_{N}(u)$ given above remains valid, and so does (2.10) after interpreting $\Gamma_{0}$ as the set of punctures for which the perturbed ConleyZehnder index (see (2.6) ) is even. One can interpret $c_{N}(u)$ as " $c_{1}$ of the normal bundle" when $u$ is immersed; in particular, $c_{N}(u)$ then predicts the number of zeroes for a generic section in the kernel of the linearized normal deformation operator at $u$, see e.g. Wen10b.

For curves in the symplectization $\mathbb{R} \times M$ of a contact manifold $(M, \xi=$ $\operatorname{ker} \alpha$ ), there is a further invariant related to $c_{N}(u)$ and the asymptotic defect. Let $\pi: T M \rightarrow \xi$ denote the fibrewise linear projection along the Reeb vector field. Then the nonlinear Cauchy-Riemann equation for $u$ : $\dot{\Sigma} \rightarrow \mathbb{R} \times M$ implies that $\pi \circ d u \in \mathcal{C}^{\infty}\left(\operatorname{Hom}_{\mathbb{C}}\left(T \dot{\Sigma}, u^{*} \xi\right)\right)$ locally satisfies a linear Cauchy-Riemann type equation, so zeroes of $\pi \circ d u$ are isolated and positive by the similarity principle unless $\pi \circ d u \equiv 0$. The latter is the case if and only if $u$ is a cover of a trivial cylinder, and otherwise, we define

$$
\operatorname{wind}_{\pi}(u) \geq 0
$$

to be the algebraic count of zeroes of $\pi \circ d u$. The asymptotic formula (2.7) implies that zeroes of $\pi \circ d u$ cannot accumulate near infinity, so $\operatorname{wind}_{\pi}(u)$ is always finite. It equals 0 if and only if $u=\left(u_{\mathbb{R}}, u_{M}\right): \dot{\Sigma} \rightarrow \mathbb{R} \times M$ has the property that $u_{M}: \dot{\Sigma} \rightarrow M$ is an immersion transverse to the Reeb vector field. From [HWZ95, Prop. 5.6], we have

$$
c_{N}(u)=\operatorname{wind}_{\pi}(u)+d_{0}(u) .
$$


In particular, this implies

$$
c_{N}(u) \geq d_{0}(u) \geq 0 \quad \text { and } \quad c_{N}(u) \geq \operatorname{wind}_{\pi}(u) \geq 0
$$

for any curve that is not a cover of a trivial cylinder, so $c_{N}(u)=0$ gives a homotopy-invariant sufficient condition for both the asymptotic defect and $\operatorname{wind}_{\pi}(u)$ to vanish.

2.4.4. Self-linking numbers. Let $\gamma$ be a nullhomologous transverse knot in a closed contact 3-manifold $(M, \xi)$, let $\Sigma \subset M$ be a Seifert surface and $X$ a framing of $\gamma$, i.e. a non-zero section of $\gamma^{*} \xi$. The self-linking number of $\gamma$ with respect to $X$ is then the algebraic count of intersections between $\Sigma$ and a generic push-off of $\gamma$ in the direction of $X$ :

$$
\operatorname{sl}(\gamma, X)=\left(\exp _{\gamma} X\right) \cdot \Sigma \in \mathbb{Z} .
$$

Note that this depends on $X$ up to homotopy, but not on $\Sigma$, as a different choice of Seifert surface changes $\operatorname{sl}(\gamma, X)$ by the homological intersection number of $\gamma$ with a closed 2-cycle, which vanishes since $\gamma$ is nullhomologous. Replacing $X$ with another framing changes $\operatorname{sl}(\gamma, X)$ by the relative winding of the two framings,

$$
\operatorname{sl}\left(\gamma, X_{1}\right)-\operatorname{sl}\left(\gamma, X_{2}\right)=\operatorname{wind}\left(X_{1}, X_{2}\right),
$$

where wind $\left(X_{1}, X_{2}\right) \in \mathbb{Z}$ denotes the winding number of the section $X_{1}$ along $\gamma$ in the trivialization induced by $X_{2}$. Note that the Seifert surface determines a canonical homotopy class of framings $X_{\Sigma}$ via the condition that $X_{\Sigma}$ should extend to a trivialization of $\xi$ along $\Sigma$, so with this choice we shall denote

$$
\operatorname{sl}(\gamma ; \Sigma):=\operatorname{sl}\left(\gamma, X_{\Sigma}\right)
$$

This depends on $\Sigma$ since $X_{\Sigma}$ does, but the dependence vanishes if $c_{1}(\xi)=0$.

With this definition in mind, suppose $\gamma$ is an unknotted Reeb orbit and $u=\left(u_{\mathbb{R}}, u_{M}\right): \mathbb{C} \rightarrow \mathbb{R} \times M$ is a $J$-holomorphic plane asymptotic to $\gamma$ for which $u_{M}: \mathbb{C} \rightarrow M$ is embedded. The closure of $u_{M}(\mathbb{C})$ is then a Seifert disk $\mathcal{D} \subset M$ for $\gamma$, and we claim

$$
\operatorname{sl}(\gamma ; \mathcal{D})=\operatorname{wind}\left(X_{\mathcal{D}}, e_{1}(u)\right),
$$

where $X_{\mathcal{D}}$ is the canonical framing determined by $\mathcal{D}$ as discussed above, and $e_{1}(u)$ is the nonzero eigenfunction appearing in the asymptotic formula (2.7) for the approach of $u$ to $\gamma$. Indeed, $e_{1}(u)$ gives the direction of the approach of $u$ to $\gamma$ and is thus homotopic to the Seifert framing of $\gamma$, implying $\operatorname{sl}\left(\gamma, e_{1}(u)\right)=0$, so

$$
\operatorname{sl}(\gamma ; \mathcal{D})=\operatorname{sl}\left(\gamma, X_{\mathcal{D}}\right)=\operatorname{sl}\left(\gamma, e_{1}(u)\right)+\operatorname{wind}\left(X_{\mathcal{D}}, e_{1}(u)\right)=\operatorname{wind}\left(X_{\mathcal{D}}, e_{1}(u)\right) .
$$

2.4.5. Siefring intersection theory. We recall here some useful properties of the intersection product on classes of $J$-holomorphic curves in almost complex manifolds with cylindrical ends. In [Sie11, Siefring associates to any pair of (not necessarily $J$-holomorphic) asymptotically cylindrical maps $u_{1}: \dot{\Sigma}_{1} \rightarrow \bar{W}$ and $u_{2}: \dot{\Sigma}_{2} \rightarrow \bar{W}$ with nondegenerate or Morse-Bott asymptotic orbits an integer

$$
u_{1} * u_{2} \in \mathbb{Z},
$$


which matches the homological intersection number $\left[u_{1}\right] \cdot\left[u_{2}\right]$ if both curves have no punctures, and in general has the following properties. First, the pairing is symmetric

$$
u_{1} * u_{2}=u_{2} * u_{1}
$$

and it is invariant under homotopies of asymptotically cylindrical maps with fixed asymptotic orbits; in fact, $u_{1} * u_{2}$ depends only on the asymptotic orbits of $u_{1}$ and $u_{2}$ and their relative homology classes. If both maps are $J$ holomorphic and their images are non-identical, then the relative asymptotic results of Sie08 imply that all intersections between $u_{1}$ and $u_{2}$ are isolated and contained in a compact subset, so by positivity of intersections, the algebraic count of intersections $u_{1} \cdot u_{2}$ is finite and satisfies

$$
u_{1} \cdot u_{2} \geq\left|\left\{\left(z_{1}, z_{2}\right) \in \dot{\Sigma}_{1} \times \dot{\Sigma}_{2} \mid u_{1}\left(z_{1}\right)=u_{2}\left(z_{2}\right)\right\}\right|,
$$

with equality if and only if all intersections are transverse. This is then related to $u_{1} * u_{2}$ by

$$
u_{1} * u_{2} \geq u_{1} \cdot u_{2},
$$

so the condition $u_{1} * u_{2}=0$ gives a homotopy-invariant sufficient condition for $u_{1}$ and $u_{2}$ to be disjoint. The following computation is an easy consequence of the definition (cf. [Sie11, Prop. 5.6]):

Proposition 2.10. Suppose $J \in \mathcal{J}(\alpha)$ for a contact 3-manifold $(M, \xi=$ $\operatorname{ker} \alpha$ ), and $u$ and $v$ are both $J$-holomorphic covers of the same trivial cylinder in $(\mathbb{R} \times M, J)$ over a nondegenerate Reeb orbit with even parity. Then $u * v=0$.

The intersection product also has a natural extension to holomorphic buildings such that homotopy invariance holds for all continuous deformations in the SFT topology. We will need a particular result about this extension:

Proposition 2.11. If $v=\left(v_{N_{+}}^{+}|\ldots| v_{1}^{+}\left|v_{0}\right| v_{1}^{-}|\ldots| v_{N_{-}}^{-}\right)$is a holomorphic building in a 4-dimensional completed symplectic cobordism, we have

$$
v * v \geq \sum_{j=1}^{N_{+}} v_{j}^{+} * v_{j}^{+}+v_{0} * v_{0}+\sum_{j=1}^{N_{-}} v_{j}^{-} * v_{j}^{-}+\sum_{\gamma} m(\gamma) p(\gamma),
$$

where the last sum is over all orbits $\gamma$ that occur as breaking orbits in $v$, with covering multiplicities denoted by $m(\gamma) \in \mathbb{N}$.

Proof. The existence of a formula

$$
v * v=\sum_{j=1}^{N_{+}} v_{j}^{+} * v_{j}^{+}+v_{0} * v_{0}+\sum_{j=1}^{N_{-}} v_{j}^{-} * v_{j}^{-}+Q
$$

with some error term $Q \geq 0$ is stated in [Sie11, Prop. 4.3(4)], and our lower bound on the error term can be extracted from the proof of that result. The point is the following. Using notation from [Wend, the pairing $u * w$ can be written in general as

$$
u * w=u \bullet_{\Phi} w-\sum_{(z, \zeta)} \Omega_{+}^{\Phi}\left(\gamma_{z}, \gamma_{\zeta}\right)-\sum_{(z, \zeta)} \Omega_{-}^{\Phi}\left(\gamma_{z}, \gamma_{\zeta}\right),
$$


where $u \bullet_{\Phi} w \in \mathbb{Z}$ denotes the relative intersection number of $u$ and $w$ with respect to an arbitrarily chosen asymptotic trivialization $\Phi$, the two sums are over all pairs of positive resp. negative punctures $z$ of $u$ and $\zeta$ of $w, \gamma_{z}$ and $\gamma_{\zeta}$ are the corresponding asymptotic orbits, and $\Omega_{ \pm}^{\Phi}\left(\gamma_{z}, \gamma_{\zeta}\right)$ are integers determined by the winding numbers $\alpha_{\mp}^{\Phi}\left(\gamma_{z}\right)$ and $\alpha_{\mp}^{\Phi}\left(\gamma_{\zeta}\right)$ (see [Wend, §4.2] for a precise formula). The same formula for $u * w$ is valid if $u$ and $w$ are buildings, and the relative intersection numbers are additive across levels. The difference between $v * v$ and the sum of the invariant self-intersection numbers of its levels is therefore a sum of terms of the form $\Omega_{+}^{\Phi}\left(\gamma, \gamma^{\prime}\right)+\Omega_{-}^{\Phi}\left(\gamma, \gamma^{\prime}\right)$ where $\gamma$ and $\gamma^{\prime}$ are pairs of breaking orbits of $v$. All of these terms are nonnegative, and in particular whenever $\gamma^{m}$ is a breaking orbit (with $\gamma$ denoting the underlying simply covered orbit), they include

$$
\Omega_{+}^{\Phi}\left(\gamma^{m}, \gamma^{m}\right)+\Omega_{-}^{\Phi}\left(\gamma^{m}, \gamma^{m}\right)=m \alpha_{+}^{\Phi}\left(\gamma^{m}\right)-m \alpha_{-}^{\Phi}\left(\gamma^{m}\right)=m p\left(\gamma^{m}\right) .
$$

If $u: \dot{\Sigma} \rightarrow \bar{W}$ is somewhere injective and $J$-holomorphic, then the relative asymptotic results of Sie08 also imply that it is embedded outside a compact subset, so there is a finite singularity count $\delta(u) \in \mathbb{Z}$, defined as the algebraic count of double points $\left\{\left(z_{1}, z_{2}\right) \in \dot{\Sigma} \times \dot{\Sigma} \mid u\left(z_{1}\right)=u\left(z_{2}\right)\right.$ and $\left.z_{1} \neq z_{2}\right\}$ after perturbing $u$ in a compact subset to make it immersed. Standard local results due to Micallef and White MW95 imply that $\delta(u) \geq 0$ with equality if and only if $u$ is embedded, but in contrast to the closed case, $\delta(u)$ is not generally homotopy invariant. Instead, it satisfies the generalized adjunction formula

$$
u * u=2 \delta_{\text {total }}(u)+c_{N}(u)+[\bar{\sigma}(u)-\# \Gamma],
$$

where

$$
\delta_{\text {total }}(u)=\delta(u)+\delta_{\infty}(u)
$$

includes an additional contribution $\delta_{\infty}(u) \geq 0$ counting "hidden" double points that can emerge from infinity under generic perturbations, and the term $\bar{\sigma}(u) \in \mathbb{N}$ is a sum of the spectral covering numbers (see 2.2 ) of all asymptotic orbits, hence $\bar{\sigma}(u)-\# \Gamma$ is also nonnegative. The formula implies that $\delta_{\text {total }}(u)$ is homotopy invariant, and since $\delta_{\infty}(u) \geq 0$, the condition $\delta_{\text {total }}(u)=0$ then suffices to ensure that all somewhere injective curves homotopic to $u$ are embedded. The converse is false in general: a curve can still be embedded with $\delta_{\text {total }}(u)>0$ due to hidden intersections, which can emerge from infinity under perturbations - but this can only happen if $u$ has at least one multiply covered asymptotic orbit or at least two punctures of the same sign that approach covers of the same orbit, thus giving the following useful criterion:

Lemma 2.12. If $u$ is a somewhere injective curve whose asymptotic orbits are all distinct and simple, then $\delta_{\infty}(u)=\bar{\sigma}(u)-\# \Gamma=0$.

The following is a minor improvement on a definition originating in Wen10a, Wen10b.

Definition 2.13. An asymptotically cylindrical $J$-holomorphic curve $u$ : $\dot{\Sigma} \rightarrow \bar{W}$ is called nicely embedded if it is somewhere injective and satisfies $u * u \leq 0$ and $\delta_{\text {total }}(u)=0$. 
It is clear from the above discussion that if $u$ is nicely embedded, then so is any other somewhere injective curve $u^{\prime}$ in the same component of the moduli space, and moreover, $u$ and $u^{\prime}$ must then be disjoint. Nicely embedded curves arise naturally in the study of finite energy foliations, initiated in [HWZ03. Their most important properties for our purposes are the following.

Lemma 2.14. If $u \in \mathcal{M}\left(J, \gamma^{+}, \gamma^{-}\right)$is nicely embedded then $c_{N}(u) \leq 0$ and $\operatorname{ind}(u) \leq 2$.

Proof. The first inequality follows directly from the definition and the adjunction formula (2.15), and this implies the second via (2.10).

Proposition 2.15. If $u \in \mathcal{M}\left(J, \gamma^{+}, \gamma^{-}\right)$is a nicely embedded curve with $\operatorname{ind}(u) \in\{1,2\}$, then $u$ is Fredholm regular.

Proof. Since $u$ is immersed by assumption and, by Lemma 2.14, satisfies $c_{N}(u) \leq 0$, it satisfies the criterion $\operatorname{ind}(u)>c_{N}(u)$ for automatic transversality given in Wen10b.

Proposition 2.16. Suppose $\mathcal{M}^{\text {nice }} \subset \mathcal{M}_{1}\left(J, \gamma^{+}, \gamma^{-}\right)$is an open and closed subset of the space of nicely embedded index 2 curves, equipped with the extra data of a marked point, such that all curves in $\mathcal{M}^{\text {nice }}$ represent the same relative homology class. Then $\mathcal{M}^{\text {nice }}$ is a smooth 4-manifold, and the evaluation map

$$
\text { ev }: \mathcal{M}^{\text {nice }} \rightarrow \bar{W}
$$

is an embedding onto an open subset of $\bar{W}$.

Proof. This is a mild generalization of a similar result proved in HWZ99 for planes with simply covered asymptotic orbits. We know every $u \in$ $\mathcal{M}^{\text {nice }}$ is Fredholm regular by Prop. 2.15, and $c_{N}(u)=0$ due to (2.10) and Lemma 2.14. It follows that $\mathcal{M}^{\text {nice }}$ is smooth and has dimension ind $(u)+2=$ 4 , and since $u * u \leq 0$ (which becomes $u * u=0$ when $c_{N}(u)=0$ ), invariance of the intersection number implies that no two curves in $\mathcal{M}^{\text {nice }}$ can intersect, hence ev $: \mathcal{M}^{\text {nice }} \rightarrow \bar{W}$ is injective. To see that it is also an immersion, observe that for a given curve $u_{0}: \dot{\Sigma} \rightarrow \bar{W}$ and marked point $\zeta_{0} \in \dot{\Sigma}$ with the pair $\left(u_{0}, \zeta_{0}\right)$ representing an element of $\mathcal{M}^{\text {nice }}$, the tangent space $T_{\left(u_{0}, \zeta_{0}\right)} \mathcal{M}^{\text {nice }}$ is naturally identified with the direct sum of $T_{\zeta_{0}} \dot{\Sigma}$ and the kernel of the linearized Cauchy-Riemann operator acting on the normal bundle of $u_{0}$. The condition $c_{N}\left(u_{0}\right)=0$ then implies via Wen10b, Equation (2.7)] that sections in this kernel are nowhere zero, hence the derivative of the evaluation map $\operatorname{ev}(u, \zeta)=u(\zeta)$ at $\left(u_{0}, \zeta_{0}\right)$ is injective.

Proposition 2.17. Suppose $\bar{W}$ is a symplectization $\left(\mathbb{R} \times M, d\left(e^{r} \alpha\right)\right)$ and $J \in$ $\mathcal{J}(\alpha)$. Then for any nicely embedded J-holomorphic curve $u=\left(u_{\mathbb{R}}, u_{M}\right)$ : $\dot{\Sigma} \rightarrow \mathbb{R} \times M$ that is not a trivial cylinder, the map $u_{M}: \dot{\Sigma} \rightarrow M$ is embedded.

Proof. Since $c_{N}(u) \leq 0$ by Lemma 2.14, $\operatorname{wind}_{\pi}(u)=0$ due to (2.11) and $u_{M}$ is therefore immersed and transverse to the Reeb vector field. To show that $u_{M}$ is injective, observe that any double point $u_{M}\left(z_{1}\right)=u_{M}\left(z_{2}\right)$ can be interpreted as an intersection of $u$ with one of its $\mathbb{R}$-translations $u^{c}:=$ $\left(u_{\mathbb{R}}+c, u_{M}\right)$ for some $c \in \mathbb{R}$, and $c$ must be nonzero since $\delta_{\text {total }}(u)=0$ implies that $u$ itself is embedded. By homotopy invariance of the intersection 
product, $u * u=u * u^{c} \leq 0$, so such an intersection is possible only if $u$ and $u^{c}$ are the same curve up to parametrization. But this would imply that $u$ is also equivalent to $u^{k c}$ for every $k \in \mathbb{N}$, so taking $k \rightarrow \infty$, we conclude from the asymptotically cylindrical behaviour of $u$ that its image lies in an arbitrarily small neighbourhood of a collection of trivial cylinders. This can only happen if $u$ itself is a trivial cylinder, so we have a contradiction.

Lemma 2.18. Under the assumptions of Prop. 2.17, suppose $u=\left(u_{\mathbb{R}}, u_{M}\right)$ : $\mathbb{C} \rightarrow \mathbb{R} \times M$ is a nicely embedded plane asymptotic to a simply covered orbit $\gamma$ and $\operatorname{ind}(u) \in\{1,2\}$. Then if $\mathcal{D} \subset M$ denotes the Seifert surface with interior $u_{M}(\mathbb{C})$, we have

$$
\mu_{\mathrm{CZ}}(\gamma ; \mathcal{D})= \begin{cases}2 & \text { if } \operatorname{ind}(u)=1, \\ 3 & \text { if } \operatorname{ind}(u)=2,\end{cases}
$$

and in both cases $\operatorname{sl}(\gamma ; \mathcal{D})=-1$.

Proof. If $\Phi$ is the trivialization of $\gamma^{*} \xi$ that extends over $\mathcal{D}$, then the relative $c_{1}$ term in the index formula vanishes and gives the stated relation between $\operatorname{ind}(u)$ and $\mu_{\mathrm{CZ}}^{\Phi}(\gamma)$. By (2.2) and (2.3), this implies $\alpha_{-}^{\Phi}(\gamma)=1$. Moreover, $c_{N}(u) \leq 0$ by Lemma 2.14, thus (2.12) implies that $u$ has zero asymptotic defect, so the nonzero eigenfunction $e_{1}(u)$ appearing in the asymptotic formula (2.7) satisfies

Now by (2.14),

$$
\operatorname{wind}^{\Phi}\left(e_{1}(u)\right)=\alpha_{-}^{\Phi}(\gamma)=1
$$

$$
\operatorname{sl}(\gamma ; \mathcal{D})=-\operatorname{wind}^{\Phi}\left(e_{1}(u)\right)=-1 .
$$

\section{SEED CURVES IN THE POSITIVE END}

In this section we describe the seed curves that will generate the moduli spaces required for proving Theorems 1.1, 1.2 and 1.3.

3.1. The standard sphere. The following construction is for the proofs of Theorems 1.1 and 1.3 ,

Regarding $S^{2 n-1}$ as the unit sphere in $\mathbb{C}^{n}$, fix the standard contact form $\alpha_{\text {std }}$ described in Example 2.2, along with the unique admissible complex structure $J_{\text {std }} \in \mathcal{J}\left(\alpha_{\text {std }}\right)$ on $\mathbb{R} \times S^{2 n-1}$ that restricts to $\xi_{\text {std }} \subset T S^{2 n-1} \subset \mathbb{C}^{n}$ as the standard complex structure $i$. Recall that the diffeomorphism

$$
\left(\mathbb{R} \times S^{2 n-1}, J_{\text {std }}\right) \rightarrow\left(\mathbb{C}^{n} \backslash\{0\}, i\right):(r, x) \mapsto e^{2 r} x
$$

is then biholomorphic, so we can regard holomorphic curves in $\mathbb{C}^{n} \backslash\{0\}$ as

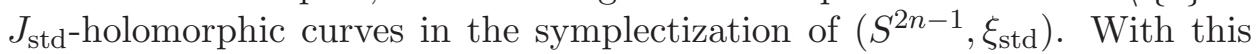
understood, define for each $w \in \mathbb{C}^{n-1} \backslash\{0\}$ the holomorphic plane

$$
u_{w}:(\mathbb{C}, i) \rightarrow\left(\mathbb{C}^{n} \backslash\{0\}, i\right): z \mapsto(z, w) .
$$

As a curve in $\mathbb{R} \times S^{2 n-1}$, each $u_{w}$ is asymptotic at $\infty$ to the same closed Reeb orbit in $\left(S^{2 n-1}, \alpha_{\text {std }}\right)$, namely

$$
\gamma_{\infty}: S^{1} \rightarrow S^{2 n-1}: t \mapsto\left(e^{2 \pi i t}, 0, \ldots, 0\right) .
$$

This orbit belongs to a $(2 n-2)$-dimensional Morse-Bott family of closed embedded Reeb orbits with period $\pi$, which foliate $S^{2 n-1}$; indeed, they form the fibres of the Hopf fibration $S^{1} \hookrightarrow S^{2 n-1} \rightarrow \mathbb{C} P^{n-1}$. 
Lemma 3.1. For each $w \in \mathbb{C}^{n-1} \backslash\{0\}$, ind $\left(u_{w}\right)=2 n-2$.

Proof. Abbreviate $\bar{W}=\mathbb{R} \times S^{2 n-1}$. The fibres of the contact bundle along $\gamma_{\infty}$ are naturally identified with $\{0\} \oplus \mathbb{C}^{n-1} \subset T S^{2 n-1} \subset \mathbb{C}^{n}$, so $\gamma_{\infty}^{*} \xi_{\text {std }}$ has a natural trivialization, which we will denote by $\Phi$, and it extends to a natural trivialization of the normal bundle $N_{u_{w}} \rightarrow \mathbb{C}$ of $u_{w}$. The latter implies $c_{1}^{\Phi}\left(N_{u_{w}}\right)=0$, so writing $u_{w}^{*} T \bar{W}=T \mathbb{C} \oplus N_{u_{w}}$ gives

$$
c_{1}^{\Phi}\left(u_{w}^{*} T \bar{W}\right)=\chi(\mathbb{C})+c_{1}^{\Phi}\left(N_{u_{w}}\right)=1 .
$$

To compute $\mu_{C Z}^{\Phi}\left(\gamma_{\infty}+\epsilon\right)$, we observe that the asymptotic operator $A_{\gamma_{\infty}}$ splits with respect to the obvious decomposition

$$
\gamma_{\infty}^{*} \xi_{\text {std }}=S^{1} \times \mathbb{C}^{n-1}=L_{2} \oplus \ldots \oplus L_{n}
$$

into trivial complex line bundles, so we can write $A_{\gamma_{\infty}}=A_{2} \oplus \ldots \oplus A_{m}$, and the trivialization $\Phi$ is now also a direct sum $\Phi_{2} \oplus \ldots \oplus \Phi_{m}$ of trivializations of these line bundles. The kernel of $A_{\gamma_{\infty}}$ is a complex $(n-1)$-dimensional space of sections along $\gamma_{\infty}$ that point in the directions of other Hopf fibres, and its intersection with each of the summands $L_{j}$ for $j=2, \ldots, n$ is a complex 1-dimensional space spanned by a section of the form

$$
\eta_{j}: S^{1} \rightarrow L_{j}: t \mapsto\left(0, \ldots, 0, e^{2 \pi i t}, 0, \ldots, 0\right) .
$$

We thus have wind $\Phi_{j}\left(\eta_{j}\right)=1$, and $A_{j}+\epsilon$ therefore has a real 2-dimensional eigenspace with the smallest positive eigenvalue $\epsilon$ and winding 1. By Proposition 2.3, the largest negative eigenvalue $A_{j}+\epsilon$ must then have winding 0 , so by (2.2),

and (2.4) then implies

$$
\mu_{C Z}^{\Phi_{j}}\left(A_{j}+\epsilon\right)=1
$$

$$
\mu_{C Z}^{\Phi}\left(\gamma_{\infty}+\epsilon\right)=\sum_{j=2}^{n} \mu_{C Z}^{\Phi_{j}}\left(A_{j}+\epsilon\right)=n-1 .
$$

Finally, (2.9) gives

$$
\begin{aligned}
\operatorname{ind}\left(u_{w}\right) & =(n-3) \chi(\mathbb{C})+2 c_{1}^{\Phi}\left(u_{w}^{*} T \bar{W}\right)+\mu_{C Z}^{\Phi}\left(\gamma_{\infty}+\epsilon\right) \\
& =(n-3)+2+(n-1)=2 n-2 .
\end{aligned}
$$

Lemma 3.2. The $J_{\text {std }}$-holomorphic planes $u_{w}$ are all Fredholm regular.

Proof. Note that the standard genericity arguments do not apply here since $J_{\text {std }}$ is very far from being generic. But in this case we can check regularity explicitly. Recall that by [Wen10b, Theorem 3], it suffices to check that the linearized normal operator

$$
\mathbf{D}_{u_{w}}^{N}: W^{1, p, \delta}\left(N_{u_{w}}\right) \rightarrow L^{p, \delta}\left(\overline{\operatorname{Hom}}_{\mathbb{C}}\left(T \mathbb{C}, N_{u_{w}}\right)\right)
$$

is surjective, where $\operatorname{ind}\left(\mathbf{D}_{u_{w}}^{N}\right)=\operatorname{ind}\left(u_{w}\right)$. Here $p \in(2, \infty)$, and $\delta>0$ is a small exponential weight, meaning that if sections $\eta: \mathbb{C} \rightarrow N_{u_{w}}$ in the domain of $\mathbf{D}_{u_{w}}^{N}$ are written near $\infty$ in cylindrical coordinates $(s, t) \in[0, \infty) \times$ $S^{1}$ corresponding to $z=e^{2 \pi(s+i t)} \in \mathbb{C}$, then the section $e^{\delta s} \eta(s, t)$ must be of class $W^{1, p}$ on $[0, \infty) \times S^{1}$. This definition also assumes a translationinvariant metric on $\mathbb{R} \times S^{2 n-1}$ for computing $L^{p}$-norms of sections along $u_{w}$. 
Note that since $p>2$, sections of class $W^{1, p}$ are continuous, and we can therefore assume

$$
\eta(s, t) \rightarrow 0 \quad \text { as } \quad s \rightarrow \infty
$$

for $\eta \in W^{1, p, \delta}\left(N_{u_{w}}\right)$.

From a different perspective, however, $\mathbf{D}_{u_{w}}^{N}$ is an extremely simple operator: sections $\eta$ of the normal bundle to $u_{w}: \mathbb{C} \rightarrow \mathbb{C}^{n} \backslash\{0\}$ can be identified canonically with functions $\tilde{\eta}: \mathbb{C} \rightarrow \mathbb{C}^{n-1}$ using the obvious trivialization of $N_{u_{w}}$, and since $\mathbf{D}_{u_{w}}^{N}$ is the linearization of the standard (and thus already linear) Cauchy-Riemann operator $\bar{\partial}, \eta \in \operatorname{ker} \mathbf{D}_{u_{w}}^{N}$ implies that $\tilde{\eta}$ is a $\mathbb{C}^{n-1}$ valued holomorphic function. Under the transformation (3.1), the condition (3.2) then implies

$$
\frac{|\tilde{\eta}(z)|}{|z|} \rightarrow 0 \quad \text { as } \quad z \rightarrow \infty
$$

so the growth of $\tilde{\eta}$ at infinity is strictly smaller than that of an affine function. Complex analysis then implies that the singularity of $\tilde{\eta}$ at $\infty$ is removable, so $\tilde{\eta}$ is constant, proving

$$
\operatorname{dim}_{\mathbb{C}} \operatorname{ker} \mathbf{D}_{u_{w}}^{N}=n-1 .
$$

The real dimension of the kernel of $\mathbf{D}_{u_{w}}^{N}$ is thus equal to its index according to Lemma 3.1, so $\mathbf{D}_{u_{w}}^{N}$ has trivial cokernel.

Lemma 3.3. Up to parametrization, every asymptotically cylindrical $J_{\text {std- }}$ holomorphic curve in $\mathbb{R} \times S^{2 n-1}$ with a single positive puncture asymptotic to $\gamma_{\infty}$ and arbitrary negative punctures is either the trivial cylinder over $\gamma_{\infty}$ or one of the planes $u_{w}$.

Proof. Since no Reeb orbit in $\left(S^{3}, \alpha_{\text {std }}\right)$ has period smaller than that of $\gamma_{\infty}$, any curve $u: \dot{\Sigma} \rightarrow \mathbb{R} \times S^{2 n-1}$ of the specified type with a nonempty set of negative punctures would satisfy $\int_{\dot{\Sigma}} u^{*} \alpha_{\text {std }}=0$ by Stokes' theorem, and since the positive asymptotic orbit is simple, $u$ in this case could only be a trivial cylinder. If $u$ has no negative punctures, then it defines via (3.1) a proper holomorphic map $u=\left(u_{1}, \ldots, u_{n}\right): \dot{\Sigma} \rightarrow \mathbb{C}^{n}$ such that $u_{2}, \ldots, u_{n}: \dot{\Sigma} \rightarrow \mathbb{C}$ are all bounded holomorphic functions that decay to 0 at the unique puncture, so these all extend to holomorphic functions on the compact domain $\Sigma$ and are therefore constant. The remaining function $u_{1}: \dot{\Sigma} \rightarrow \mathbb{C}$ has a pole of order 1 at its unique puncture, thus it extends to a nonconstant holomorphic map $\Sigma \rightarrow S^{2}$ of degree 1, implying that $\Sigma=S^{2}$ and, after a suitable reparametrization, $\dot{\Sigma}=\mathbb{C}$ with $u_{1}: \mathbb{C} \rightarrow \mathbb{C}$ an affine map.

Lemma 3.4. In the case $\operatorname{dim} M=3$, the planes $u_{w}$ satisfy $c_{N}\left(u_{w}\right)=0$ and are nicely embedded.

Proof. We saw in the proof of Lemma 3.1 that $\mu_{C Z}^{\Phi}\left(\gamma_{\infty}+\epsilon\right)$ is odd and $\operatorname{ind}\left(u_{w}\right)=2$, so (2.10) implies $c_{N}\left(u_{w}\right)=0$. Since $u_{w}$ is embedded and has only a single simple asymptotic orbit, $\delta_{\text {total }}\left(u_{w}\right)=\bar{\sigma}\left(u_{w}\right)-1=0$ by Lemma 2.12. Thus by Siefring's adjunction formula (2.15),

$$
u_{w} * u_{w}=2 \delta_{\text {total }}\left(u_{w}\right)+c_{N}\left(u_{w}\right)+\left[\bar{\sigma}\left(u_{w}\right)-1\right]=0 .
$$


3.2. Reducible tight contact 3-manifolds. We now describe the seed curves for the first case of Theorem 1.2. Assume $M$ is a reducible closed oriented 3-manifold with a contact structure $\xi$; we are free to assume $\xi$ is tight since the overtwisted case will be dealt with separately in 3.3 below. The reducibility hypothesis means that $M$ is either $S^{1} \times S^{2}$ or a nontrivial connected sum $M_{1} \# M_{2}$, and in the latter case, tightness of $\xi$ implies via Colin's connected sum theorem [Col97] that $(M, \xi)=\left(M_{1}, \xi_{1}\right) \#\left(M_{2}, \xi_{2}\right)$ for some tight contact structures $\xi_{i}$ on $M_{i}, i=1,2$. The case $S^{1} \times S^{2}$ can also be understood via connected sums since the unique tight contact structure on $S^{1} \times S^{2}$ is the one that is obtained from $\left(S^{3}, \xi_{\text {std }}\right)$ by attaching two disjoint neighborhoods in $S^{3}$ to each other via a self-connected sum. In either case, $(M, \xi)$ contains a special embedded 2-sphere

$$
S^{2} \cong S \subset M,
$$

the belt sphere of the connected sum, and $\xi$ takes a certain standard form in a neighbourhood of $S$. Moreover, $S$ represents a nontrivial element of $\pi_{2}(M)$ : this follows from the Poincaré conjecture after applying [Hat, Prop. 3.1] to deduce that $[S] \in \pi_{2}(M)$ can be trivial only if $S$ bounds a contractible submanifold in $M$.

The desired $J$-holomorphic curves in $\mathbb{R} \times M$ can now be borrowed wholesale from a construction of Fish and Siefring [FS18. Specifically, Theorem 5.1 in their paper provides a nondegenerate contact form $\alpha_{+}$on $(M, \xi)$ and an almost complex structure $J_{+} \in \mathcal{J}\left(\alpha_{+}\right)$, which may be assumed generic outside a neighborhood of $\mathbb{R} \times S$, such that there exists a nondegenerate embedded Reeb orbit

$$
\gamma_{\infty}: S^{1} \rightarrow M
$$

with even Conley-Zehnder index and with image in $S$. This orbit splits $S$ into two hemispheres $S_{+}$and $S_{-}$, and there exists a pair of nicely embedded and Fredholm regular $J_{+}$-holomorphic planes

$$
u^{ \pm}=\left(u_{\mathbb{R}}^{ \pm}, u_{M}^{ \pm}\right): \mathbb{C} \rightarrow \mathbb{R} \times M
$$

with index 1 , both asymptotic to $\gamma_{\infty}$, such that $u_{M}^{ \pm}(\mathbb{C})$ is the interior of $S_{ \pm}$. They satisfy

$$
c_{N}\left(u^{ \pm}\right)=u^{ \pm} * u^{ \pm}=u^{+} * u^{-}=0,
$$

and they approach $\gamma_{\infty}$ "from opposite sides" in the sense that after suitable $\mathbb{R}$-translations, one can arrange

$$
e_{1}\left(u^{+}\right)=-e_{1}\left(u^{-}\right),
$$

where $e_{1}\left(u^{ \pm}\right)$denotes the nontrivial asymptotic eigenfunction appearing in the asymptotic formula (2.7) for $u^{ \pm}$.

Lemma 3.5. Up to parametrization and $\mathbb{R}$-translation, every asymptotically cylindrical $J_{+}$-holomorphic curve in $\mathbb{R} \times M$ with a single positive puncture asymptotic to $\gamma_{\infty}$ and arbitrary negative punctures is either the trivial cylinder over $\gamma_{\infty}$ or one of the planes $u^{ \pm}$.

Proof. We use Siefring's intersection theory. Fix a trivialization $\Phi$ of $\xi$ along $\gamma_{\infty}$. The first observation is that since $d_{0}\left(u^{ \pm}\right) \leq c_{N}\left(u^{ \pm}\right)=0$, the 
eigenfunctions $e_{1}\left(u^{ \pm}\right)$both have maximal winding $\alpha_{-}^{\Phi}\left(\gamma_{\infty}\right)$. Since $\mu_{C Z}^{\Phi}\left(\gamma_{\infty}\right)$ is even, Proposition 2.3 implies that there is only a 1-dimensional eigenspace

$$
E_{\lambda} \subset \mathcal{C}^{\infty}\left(\gamma_{\infty}^{*} \xi\right)
$$

of $A_{\gamma_{\infty}}$ with negative eigenvalue and winding $\alpha_{-}^{\Phi}\left(\gamma_{\infty}\right)$, and $e_{1}\left(u^{ \pm}\right) \in E_{\lambda}$. Denoting the trivial cylinder over $\gamma_{\infty}$ by $\mathbb{R} \times \gamma_{\infty}$, this implies

$$
u^{ \pm} *\left(\mathbb{R} \times \gamma_{\infty}\right)=0 .
$$

Indeed, there are no geometric intersections between $u^{ \pm}$and $\mathbb{R} \times \gamma_{\infty}$ since $u_{M}^{ \pm}(\mathbb{C})$ is the interior of $S_{ \pm}$, but one must still rule out asymptotic contributions, i.e. "hidden" intersections at infinity. These are characterized in Sie11] in terms of relative winding numbers, and in the present situation, the asymptotic contribution to $u^{ \pm} *\left(\mathbb{R} \times \gamma_{\infty}\right)$ vanishes if and only if the asymptotic representative describing the approach of $u^{ \pm}$to $\mathbb{R} \times \gamma_{\infty}$ at infinity has maximal winding. This is true since wind ${ }^{\Phi}\left(e_{1}\left(u^{ \pm}\right)\right)=\alpha_{-}^{\Phi}\left(\gamma_{\infty}\right)$.

Now suppose $u: \dot{\Sigma} \rightarrow \mathbb{R} \times M$ is a $J_{+}$-holomorphic curve with the specified properties. We claim

$$
u * u^{ \pm}=0 .
$$

To see this, first use $\mathbb{R}$-translation to move $u^{ \pm}$until its image is contained in $[0, \infty) \times M$, which is possible since $u^{ \pm}$has no negative punctures. Then notice that since $u$ has only one positive puncture and it is asymptotic to $\gamma_{\infty}, u$ admits a homotopy through asymptotically cylindrical (but not necessarily $J_{+}$-holomorphic) maps to a map whose intersection with $[0, \infty) \times$ $M$ is identical to the top half of the trivial cylinder $\mathbb{R} \times \gamma_{\infty}$. Using the homotopy invariance of the intersection product, it follows that $u * u^{ \pm}=$ $u^{ \pm} *\left(\mathbb{R} \times \gamma_{\infty}\right)=0$.

Finally, let $e_{1}(u)$ denote the nontrivial asymptotic eigenfunction in (2.7) that controls the approach of $u$ to $\gamma_{\infty}$ at its positive puncture. If $e_{1}(u) \notin E_{\lambda}$, then $\operatorname{wind}^{\Phi}\left(e_{1}(u)\right)<\alpha_{-}^{\Phi}\left(\gamma_{\infty}\right)=\operatorname{wind}^{\Phi}\left(e_{1}\left(u^{ \pm}\right)\right)$. In this case the projections of $u$ and $u^{ \pm}$to $M$ obviously intersect each other near $\gamma_{\infty}$, implying that some $\mathbb{R}$-translation of $u$ intersects $u^{ \pm}$, but this is impossible by (3.3). We therefore have $e_{1}(u) \in E_{\lambda}$. But observe now that applying $\mathbb{R}$-translations to $u$ modifies $e_{1}(u)$ by multiplication with a positive constant, so since $\operatorname{dim} E_{\lambda}=1$ and $e_{1}\left(u^{+}\right)$and $e_{1}\left(u^{-}\right)$have opposite signs, there exists a unique $\mathbb{R}$-translation for which $e_{1}(u)$ precisely matches either $e_{1}\left(u^{+}\right)$or $e_{1}\left(u^{-}\right)$. For concreteness, suppose $e_{1}(u)=e_{1}\left(u^{+}\right)$. Then the main results of Sie08 imply that unless $u=u^{+}$up to parametrization, there is a nontrivial asymptotic eigenfunction controlling the approach of $u$ to $u^{+}$at their positive ends, and it lies in a different eigenspace, with winding strictly less than $\alpha_{-}^{\Phi}\left(\gamma_{\infty}\right)$. The characterization of asymptotic contributions in Sie11] then implies that $u * u^{+}>0$, again contradicting (3.3).

Remark 3.6. The above lemma does not specifically require the manifold $M$ to be reducible: it only requires the existence of a simple Reeb orbit $\gamma_{\infty}$ spanned by two disjoint embedded index 1 holomorphic planes $u^{ \pm}$that approach $\gamma_{\infty}$ "from opposite sides" in the sense described above. The conditions $c_{N}\left(u^{ \pm}\right)=u^{ \pm} * u^{ \pm}=u^{+} * u^{-}=0$ follow automatically from these assumptions via (2.10) and Siefring's adjunction formula (2.15). 
3.3. Overtwisted contact 3-manifolds. If $(M, \xi)$ is overtwisted, then Eliashberg's appendix to Yau06] uses the following geometric picture to prove vanishing of contact homology. There is a nondegenerate contact form $\alpha_{+}$and an almost complex structure $J_{+} \in \mathcal{J}\left(\alpha_{+}\right)$admitting an embedded Fredholm regular $J_{+}$-holomorphic plane

$$
u^{\infty}=\left(u_{\mathbb{R}}^{\infty}, u_{M}^{\infty}\right): \mathbb{C} \rightarrow \mathbb{R} \times M
$$

with index 1 , asymptotic to a simple Reeb orbit

$$
\gamma_{\infty}: S^{1} \rightarrow M
$$

with even Conley-Zehnder index, such that $u^{\infty}$ is (up to parametrization and $\mathbb{R}$-translation) the only nontrivial $J_{+}$-holomorphic curve in $\mathbb{R} \times M$ with one positive puncture asymptotic to $\gamma_{\infty}$ (and arbitrary negative punctures). A more detailed version of this construction can be extracted as a special case from Wen13a], using intersection-theoretic arguments similar to those of 3.2 above. Since $u^{\infty}$ is asymptotic to a simple orbit, Lemma 2.12 implies $\delta_{\infty}\left(u^{\infty}\right)=\bar{\sigma}\left(u^{\infty}\right)-1=0$, and since it is also embedded, $\delta_{\text {total }}\left(u^{\infty}\right)=0$. Moreover, by (2.10), $c_{N}\left(u^{\infty}\right)=0$, so the adjunction formula (2.15) now gives

$$
u^{\infty} * u^{\infty}=2 \delta_{\text {total }}\left(u^{\infty}\right)+c_{N}\left(u^{\infty}\right)+\left[\bar{\sigma}\left(u^{\infty}\right)-1\right]=0,
$$

implying that $u^{\infty}$ is nicely embedded.

\section{A LOCAL ADJUNCTION FORMULA FOR BREAKING HOLOMORPHIC ANNULI}

The aim of this section is to prove Theorem 1.16 and derive Corollary 1.17. We assume throughout that $M$ is a 3-manifold endowed with $\mathcal{C}^{\infty}$ converging sequences of contact forms $\alpha_{k} \rightarrow \alpha_{\infty}$ and admissible almost complex structures $J_{k} \in \mathcal{J}\left(\alpha_{k}\right), k \leq \infty$. Fix a nondegenerate closed Reeb orbit $\gamma: S^{1} \rightarrow M$ for $\alpha_{\infty}$ with covering multiplicity $m \in \mathbb{N}$, period $T>0$ and parity $p(\gamma) \in\{0,1\}$. We consider a sequence of $J_{k}$-holomorphic annuli

$$
u_{k}:\left(\left[-R_{k}, R_{k}^{\prime}\right] \times S^{1}, i\right) \rightarrow\left(\mathbb{R} \times M, J_{k}\right),
$$

where $R_{k}, R_{k}^{\prime} \rightarrow \infty$ and $u_{k}$ converges in the SFT topology to a broken $J_{\infty}$-holomorphic annulus

$$
u_{k} \rightarrow\left(u_{\infty}^{+} \mid u_{\infty}^{-}\right)
$$

in which both levels are embedded and $\gamma$ is the breaking orbit. More precisely, $u_{\infty}^{ \pm}$are embedded $J_{\infty}$-holomorphic half-cylinders

$$
u_{\infty}^{-}:[0, \infty) \times S^{1} \rightarrow \mathbb{R} \times M, \quad u_{\infty}^{+}:(-\infty, 0] \times S^{1} \rightarrow \mathbb{R} \times M
$$

with

$$
u_{\infty}^{ \pm}(s, t)=\exp _{(T s, \gamma(t))} h_{ \pm}(s, t)
$$

for some translation-invariant metric on $\mathbb{R} \times M$ and asymptotic representatives satisfying $h_{ \pm}(s, \cdot) \rightarrow 0$ with all derivatives as $s \rightarrow \mp \infty$. To say what the convergence $u_{k} \rightarrow\left(u_{\infty}^{+} \mid u_{\infty}^{-}\right)$means, denote the $\mathbb{R}$-translation action on $\mathbb{R} \times M$ by

$$
\tau_{c}: \mathbb{R} \times M \rightarrow \mathbb{R} \times M:(r, x) \mapsto(r+c, x)
$$

for $c \in \mathbb{R}$. Then we require

$$
\tau_{r_{k}} \circ u_{k}\left(\cdot+R_{k}^{\prime}, \cdot\right) \rightarrow u_{\infty}^{+} \quad \text { in } \quad \mathcal{C}_{\mathrm{loc}}^{\infty}\left((-\infty, 0] \times S^{1}, \mathbb{R} \times M\right)
$$


for some sequence $r_{k} \rightarrow-\infty$, while

$$
\tau_{r_{k}} \circ u_{k}\left(\cdot-R_{k}, \cdot\right) \rightarrow u_{\infty}^{-} \quad \text { in } \quad \mathcal{C}_{\mathrm{loc}}^{\infty}\left([0, \infty) \times S^{1}, \mathbb{R} \times M\right)
$$

for some sequence $r_{k} \rightarrow+\infty$. Additionally, choose diffeomorphisms $\varphi_{-}$: $[-1,0) \rightarrow[0, \infty)$ and $\varphi_{+}:(0,1] \rightarrow(-\infty, 0]$, let $\pi_{M}: \mathbb{R} \times M \rightarrow M$ denote the natural projection, and define the continuous map

$$
\bar{u}_{\infty}^{M}:[-1,1] \times S^{1} \rightarrow M:(s, t) \mapsto \begin{cases}\pi_{M} \circ u_{\infty}^{+}\left(\varphi_{+}(s), t\right) & \text { if } s>0 \\ \gamma(t) & \text { if } s=0, \\ \pi_{M} \circ u_{\infty}^{-}\left(\varphi_{-}(s), t\right) & \text { if } s<0\end{cases}
$$

We then also require the existence of a sequence of diffeomorphisms $\varphi_{k}$ : $[-1,1] \times S^{1} \rightarrow\left[-R_{k}, R_{k}^{\prime}\right] \times S^{1}$ such that

$$
\pi_{M} \circ u_{k} \circ \varphi_{k} \rightarrow \bar{u}_{\infty}^{M} \quad \text { in } \quad C^{0}\left([-1,1] \times S^{1}, M\right) .
$$

With these hypotheses understood, the statement of Theorem 1.16 is that for all $k$ sufficiently large,

$$
2 \delta\left(u_{k}\right)=2\left[\delta_{\infty}\left(u_{\infty}^{+}\right)+\delta_{\infty}\left(u_{\infty}^{-}\right)\right]+\left[\bar{\sigma}_{+}(\gamma)-1\right]+\left[\bar{\sigma}_{-}(\gamma)-1\right]+(m-1) p(\gamma) .
$$

The proof is based on a relative adjunction formula in the style of Hutchings [Hut02]. Since $u_{\infty}^{+}$and $u_{\infty}^{-}$are embedded, we are free to assume $u_{k}$ is embedded near the boundary of its domain for sufficiently large $k$; moreover, if we reparametrize $u_{\infty}^{ \pm}$by suitable shifts to focus only on neighborhoods of $\pm \infty$, the corresponding adjustments in $u_{k}$ can be arranged so that its tangent spaces are close to those of a trivial cylinder for large $k$. This means replacing the domains $\left[-R_{k}, R_{k}^{\prime}\right] \times S^{1}$ of $u_{k}$ with smaller domains that nonetheless still expand to infinite length, and we do not lose any singularities this way since $u_{\infty}^{ \pm}$are both embedded on the corresponding portions of their domains that are being discarded. With this understood, if we choose a trivialization $\Phi$ of $\xi=\operatorname{ker} \alpha_{\infty}$ along $\gamma$, this determines a trivialization of the normal bundle of $u_{k}$ along its boundary uniquely up to homotopy for large $k$. Define the relative self-intersection number of $u_{k}$,

$$
u_{k} \bullet_{\Phi} u_{k} \in \mathbb{Z}
$$

as the algebraic count of intersections between $u_{k}$ and a generic perturbation of $u_{k}$ that is pushed in the direction of $\Phi$ near the boundary. This number depends on $\Phi$ up to homotopy. We can similarly define $u_{\infty}^{ \pm} \bullet_{\Phi} u_{\infty}^{ \pm}$with the condition that the second copy of $u_{\infty}^{ \pm}$is pushed by some small but nonzero amount in the direction of $\Phi$ both near its boundary and near infinity. The convergence $u_{k} \rightarrow\left(u_{\infty}^{+} \mid u_{\infty}^{-}\right)$then implies

$$
u_{k} \bullet_{\Phi} u_{k}=u_{\infty}^{+} \bullet_{\Phi} u_{\infty}^{+}+u_{\infty}^{-} \bullet_{\Phi} u_{\infty}^{-}
$$

for sufficiently large $k$. The relative adjunction formula relates these selfintersection numbers to the count of double points and corresponding relative first Chern numbers

$$
c_{1}^{\Phi}\left(u_{k}^{*} T(\mathbb{R} \times M)\right), c_{1}^{\Phi}\left(\left(u_{\infty}^{ \pm}\right)^{*} T(\mathbb{R} \times M)\right) \in \mathbb{Z},
$$

defined by regarding $\Phi$ as a trivialization of the normal bundle of $u_{k}$ or $u_{\infty}^{ \pm}$ over the boundary and/or near infinity - this sums with the canonical parallelization of the domains (annuli and half-cylinders) to give trivializations 
of the pulled back tangent bundle on these regions. Appealing again to the convergence $u_{k} \rightarrow\left(u_{\infty}^{+} \mid u_{\infty}^{-}\right)$, we have

$$
c_{1}^{\Phi}\left(u_{k}^{*} T(\mathbb{R} \times M)\right)=c_{1}^{\Phi}\left(\left(u_{\infty}^{+}\right)^{*} T(\mathbb{R} \times M)\right)+c_{1}^{\Phi}\left(\left(u_{\infty}^{-}\right)^{*} T(\mathbb{R} \times M)\right)
$$

for large $k$. Note that if $u_{k}$ is immersed with normal bundle $N_{u_{k}}$, then $c_{1}^{\Phi}\left(u_{k}^{*} T(\mathbb{R} \times M)\right)=c_{1}^{\Phi}\left(N_{u_{k}}\right)$ since the domains have vanishing Euler characteristic. If $u_{k}$ is not immersed, one can perturb it to an immersion without changing $c_{1}^{\Phi}\left(u_{k}^{*} T(\mathbb{R} \times M)\right)$, so the same argument used to prove the adjunction formula for closed holomorphic curves gives the relative formula

$$
u_{k} \bullet u_{k}=2 \delta\left(u_{k}\right)+c_{1}^{\Phi}\left(u_{k}^{*} T(\mathbb{R} \times M)\right) .
$$

This makes crucial use of the fact that $u_{k}$ is embedded near the boundary for large $k$, so pushing it in normal directions determined by $\Phi$ does not produce any intersections near the boundary.

The analogous story for $u_{\infty}^{ \pm}$is slightly more complicated if $\gamma$ has covering multiplicity $m>1$, because new intersections can appear near infinity after pushing off via $\Phi$. This phenomenon was observed in [Hut02, §3.2] and quantified in terms of the writhe of a braid determined by the asymptotic behaviour of $u_{\infty}^{ \pm}$. Using notation adapted from [Sie11, §3.2] (see also [Wend, $\S 4.3]$ ), we denote by

$$
i_{\infty}^{\Phi}\left(u_{\infty}^{ \pm}\right) \in \mathbb{Z}
$$

the algebraic count of intersections near infinity between $u_{\infty}^{ \pm}$and a small perturbation of itself via $\Phi$. This number matches the writhe described in Hut02 up to a sign. It is only nonzero if $m \geq 2$, and in that case it depends on $\Phi$ up to homotopy; in [Sie11] it is expressed as a sum of winding numbers of asymptotic eigenfunctions that control the relative approach of different parametrizations of $u_{\infty}^{ \pm}$near the orbit. The bounds on these winding numbers coming from Proposition 2.3 lead to the bound

$$
i_{\infty}^{\Phi}\left(u_{\infty}^{ \pm}\right) \geq \Omega_{\mp}^{\Phi}(\gamma):= \pm(m-1) \alpha_{ \pm}^{\Phi}(\gamma)+\left[\bar{\sigma}_{ \pm}(\gamma)-1\right],
$$

which furnishes the definition of the number $\delta_{\infty}\left(u_{\infty}^{ \pm}\right)$counting "hidden" double points at infinity:

$$
\delta_{\infty}\left(u_{\infty}^{ \pm}\right)=\frac{1}{2}\left[i_{\infty}^{\Phi}\left(u_{\infty}^{ \pm}\right)-\Omega_{\mp}^{\Phi}(\gamma)\right] \geq 0
$$

Including the contribution from intersections near infinity, the relative adjunction formula for $u_{\infty}^{ \pm}$takes the form

$$
u_{\infty}^{ \pm} \bullet u_{\infty}^{ \pm}=2 \delta\left(u_{\infty}^{ \pm}\right)+c_{1}^{\Phi}\left(\left(u_{\infty}^{ \pm}\right)^{*} T(\mathbb{R} \times M)\right)+i_{\infty}^{\Phi}\left(u_{\infty}^{ \pm}\right) .
$$

We are now ready to prove both the theorem and the corollary.

Proof of Theorem 1.16. We can use the various relative adjunction formulas to rewrite both the left and right hand sides of (4.1), thus

$$
\begin{aligned}
2 \delta\left(u_{k}\right)+c_{1}^{\Phi}\left(u_{k}^{*} T(\mathbb{R} \times M)\right) & =2 \delta\left(u_{\infty}^{+}\right)+c_{1}^{\Phi}\left(\left(u_{\infty}^{+}\right)^{*} T(\mathbb{R} \times M)\right)+i_{\infty}^{\Phi}\left(u_{\infty}^{+}\right) \\
& +2 \delta\left(u_{\infty}^{-}\right)+c_{1}^{\Phi}\left(\left(u_{\infty}^{-}\right)^{*} T(\mathbb{R} \times M)\right)+i_{\infty}^{\Phi}\left(u_{\infty}^{-}\right) .
\end{aligned}
$$

The terms $\delta\left(u_{\infty}^{ \pm}\right)$vanish since $u_{\infty}^{+}$and $u_{\infty}^{-}$are embedded, and combining this with (4.2) gives

$$
2 \delta\left(u_{k}\right)=i_{\infty}^{\Phi}\left(u_{\infty}^{+}\right)+i_{\infty}^{\Phi}\left(u_{\infty}^{-}\right)
$$


Now plugging in (4.4) and $p(\gamma)=\alpha_{+}^{\Phi}(\gamma)-\alpha_{-}^{\Phi}(\gamma)$ gives the stated formula.

Proof of Corollary 1.17. The local adjunction formula implies that if $\delta\left(u_{k}\right)=$ 0 , then

(1) $\delta_{\infty}\left(u_{\infty}^{+}\right)=\delta_{\infty}\left(u_{\infty}^{-}\right)=0$

(2) $\bar{\sigma}_{+}(\gamma)=\bar{\sigma}_{-}(\gamma)=1$, and

(3) $\gamma$ is either simply covered or has even parity.

In the case with even parity, we can derive further constraints on the multiplicity $m$ from the condition on the spectral covering numbers: recalling Proposition 2.3, the extremal winding numbers $\alpha_{ \pm}^{\Phi}(\gamma)$ for positive and negative eigenvalues match, and $\bar{\sigma}_{ \pm}(\gamma)=1$ means that any nontrivial eigenfunction in the corresponding eigenspaces $E_{ \pm}$is simply covered. Both of these eigenspaces are also 1-dimensional, and since $\gamma$ has multiplicity $m$, there is a linear $\mathbb{Z}_{m}$-action on each $E_{ \pm}$generated by the map that sends an eigenfunction $e \in E_{ \pm}$to $e(\cdot+1 / m)$. This defines a real 1-dimensional representation of $\mathbb{Z}_{m}$, and the representation must be faithful since $E_{ \pm}$contains simply covered eigenfunctions. Real 1-dimensional representations of finite groups can act only by \pm 1 , so the only possibility for $m>1$ is that $m=2$ and the generator of $\mathbb{Z}_{2}$ acts by -1 . We claim finally that in this case, the underlying simple orbit has odd parity. Indeed, Proposition 2.3 implies that it would otherwise have two eigenfunctions with the same winding but eigenvalues of opposite sign, and $E_{ \pm}$would then have to consist of the double covers of those eigenfunctions, which is a contradiction.

\section{CompaCtness for NiCELy EMBedded Planes in COBORdisms}

In this section we fix the following assumptions. Let $(W, d \lambda)$ denote a 4 dimensional Liouville cobordism with concave boundary $\left(M_{-}, \xi_{-}=\operatorname{ker} \alpha_{-}\right)$ and convex boundary $\left(M_{+}, \xi_{+}=\operatorname{ker} \alpha_{+}\right)$, where $\left.\lambda\right|_{T M_{ \pm}}=\alpha_{ \pm}$, with $\alpha_{-}$ assumed nondegenerate and $\alpha_{+}$Morse-Bott. The symplectic completion of $W$ will be denoted as usual by $\bar{W}$, and we choose $J \in \mathcal{J}\left(W, \omega, \alpha_{+}, \alpha_{-}\right)$to be generic in the interior of $W$ such that its restriction $J_{-}$to the negative end is also generic; in particular, this means that all simple $J$-holomorphic curves in $\bar{W}$ that pass through the interior of $W$ have nonnegative index, and all simple $J_{-}$-holomorphic curves in $\mathbb{R} \times M_{-}$other than trivial cylinders have index at least 1 . Fix a simply covered Reeb orbit

$$
\gamma_{\infty}: S^{1} \rightarrow M_{+}
$$

The main objective of this section is the following theorem, which characterizes the closure in the SFT compactification of the set of planes in $\mathcal{M}\left(J, \gamma_{\infty}, \emptyset\right)$ that are nicely embedded in the sense of Definition 2.13. For application to our main theorems, the results of 33 permit us to ignore holomorphic buildings with nontrivial upper levels.

Theorem 5.1. Suppose $u_{k} \in \mathcal{M}\left(J, \gamma_{\infty}, \emptyset\right)$ is a sequence of nicely embedded planes converging in the sense of $\left[\mathrm{BEH}^{+} 03\right]$ to a holomorphic building $u_{\infty} \in$ $\overline{\mathcal{M}}\left(J, \gamma_{\infty}, \emptyset\right)$ with no nontrivial upper levels but at least one nontrivial lower level. Then all components of the levels of $u_{\infty}$ other than trivial cylinders are nicely embedded, all breaking orbits are either simply covered or are 

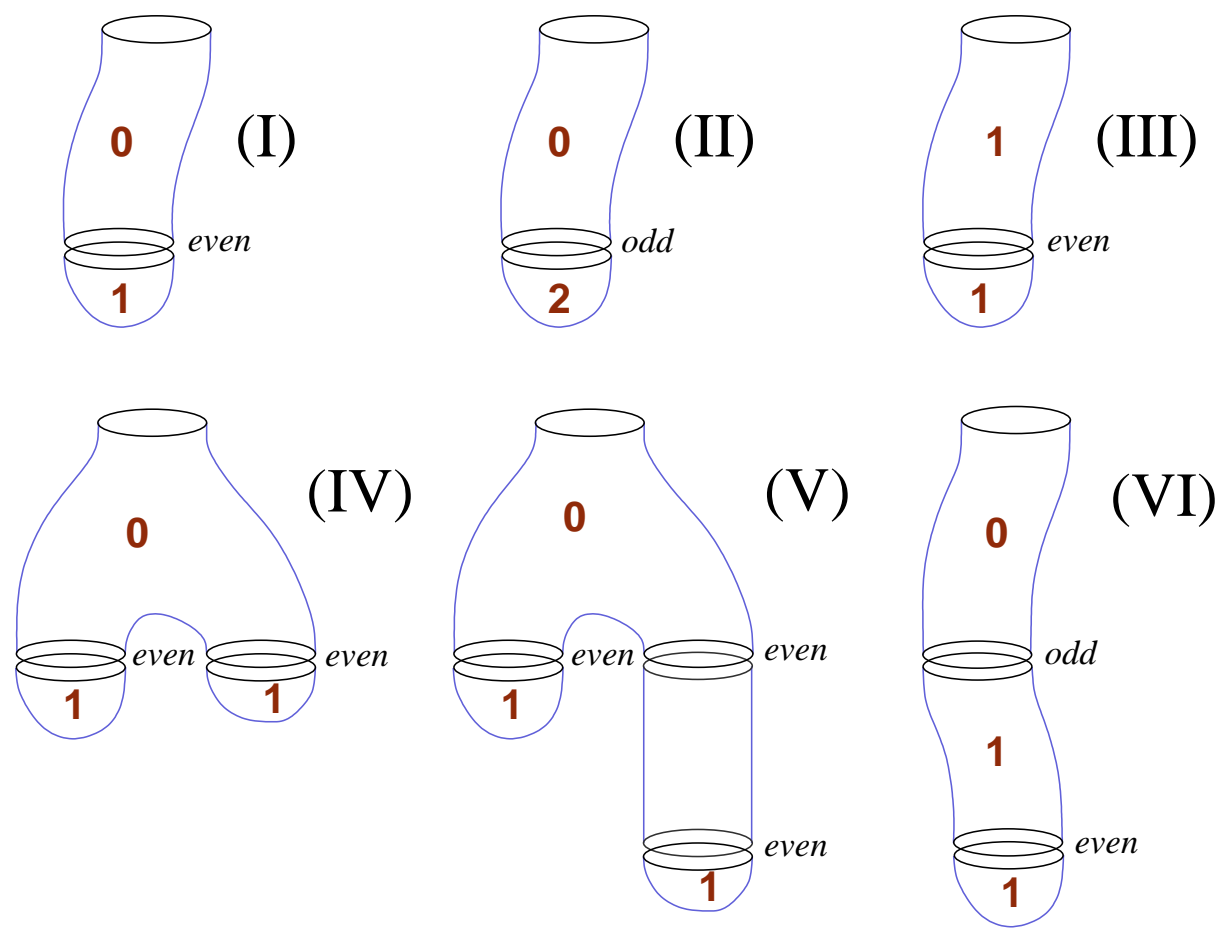

Figure 5. The six types of holomorphic buildings in Theorem 5.1.

doubly covered bad orbits, and $u_{\infty}$ fits one of the following descriptions (see Figure (5):

- Type (I): $\left(v_{0} \mid v_{1}^{-}\right)$, where $v_{0}$ is an index 0 cylinder, $v_{1}^{-}$is an index 1 plane, and the breaking orbit has even parity.

- Type (II): $\left(v_{0} \mid v_{1}^{-}\right)$, where $v_{0}$ is an index 0 cylinder, $v_{1}^{-}$is an index 2 plane, and the breaking orbit has odd parity.

- Type (III): $\left(v_{0} \mid v_{1}^{-}\right)$, where $v_{0}$ is an index 1 cylinder, $v_{1}^{-}$is an index 1 plane, and the breaking orbit has even parity.

- Type (IV): $\left(v_{0} \mid v_{1}^{-}\right)$, where $v_{0}$ has index 0 and two negative punctures, $v_{1}^{-}$is a disjoint union of two index 1 planes, and both breaking orbits have even parity.

- Type $(V):\left(v_{0}\left|v_{1}^{-}\right| v_{2}^{-}\right)$, where $v_{0}$ has index 0 and two negative punctures, $v_{1}^{-}$is the disjoint union of a trivial cylinder with an index 1 plane, and $v_{2}^{-}$is an additional index 1 plane, with all breaking orbits having even parity.

- Type (VI): $\left(v_{0}\left|v_{1}^{-}\right| v_{2}^{-}\right)$, where $v_{0}$ is an index 0 cylinder, $v_{1}^{-}$is an index 1 cylinder and $v_{2}^{-}$is an index 1 plane, the breaking orbit between $v_{0}$ and $v_{1}^{-}$has odd parity, and the breaking orbit between $v_{1}^{-}$and $v_{2}^{-}$ has even parity.

We begin with a few preliminary observations that will be used repeatedly in the proof. Recall that by Prop. 2.5. $u_{\infty}$ must have the structure of a tree, with all connected components having exactly one positive puncture and the bottom level being a disjoint union of capping planes. The top of this tree is 
the main level, which will always be somewhere injective since $\gamma_{\infty}$ is a simply covered orbit. Moreover, since $J$ is generic, Equation 2.10 and Lemma 2.14 imply that the curves $u_{k}$ in our sequence can be assumed to satisfy either $\operatorname{ind}\left(u_{k}\right) \in\{1,2\}$ and $c_{N}\left(u_{k}\right)=0$ or $\operatorname{ind}\left(u_{k}\right)=0$ and $c_{N}\left(u_{k}\right)=-1$. Note that all the building types in the above theorem have total index 2 except for Type (I), which occurs in the index 1 case. We shall denote

$$
u_{\infty}=\left(v_{0}\left|v_{1}^{-}\right| \ldots \mid v_{N}^{-}\right)
$$

where by assumption $N \geq 1$, and each $v_{j}^{-}$is in general a disjoint union of $m_{j} \geq 1$ connected curves

$$
v_{j, 1}^{-}, \ldots, v_{j, m_{j}}^{-}
$$

each having exactly one positive puncture. The definition of the normal Chern number, together with the relation (2.3) between parities and winding numbers, implies the formula

$$
0 \geq c_{N}\left(u_{k}\right)=c_{N}\left(v_{0}\right)+\sum_{j=1}^{N} \sum_{i=1}^{m_{j}} c_{N}\left(v_{j, i}^{-}\right)+\sum_{j=1}^{N} \sum_{i=1}^{m_{j}} p\left(\gamma_{j, i}\right),
$$

where $\gamma_{j, i}$ denotes the asymptotic orbit at the unique positive puncture of $v_{j, i}^{-}$, i.e. the $\gamma_{j, i}$ are all the breaking orbits of $u_{\infty}$. Since $u_{\infty}$ has no negative punctures in its lowest level, we can conveniently repackage this formula as

$$
0 \geq c_{N}\left(u_{k}\right)=\hat{c}_{N}\left(v_{0}\right)+\sum_{j=1}^{N} \sum_{i=1}^{m_{j}} \hat{c}_{N}\left(v_{j, i}^{-}\right),
$$

where for any punctured holomorphic curve $w$, we define $\hat{c}_{N}(w)$ to be the sum of $c_{N}(w)$ with the parities of the asymptotic orbits at all its negative punctures.

Lemma 5.2. All the components $v_{j, i}^{-}$in lower levels have $c_{N}\left(v_{j, i}^{-}\right)=0$, and one of the following holds:

(1) All breaking orbits in $u_{\infty}$ have even parity and the main level $v_{0}$ satisfies $c_{N}\left(v_{0}\right)=0$, or

(2) The main level $v_{0}$ is a cylinder with $c_{N}\left(v_{0}\right)=-1$ whose negative asymptotic orbit has odd parity, and all other breaking orbits have even parity.

Proof. Genericity implies ind $\left(v_{0}\right) \geq 0$, so by (2.10), $c_{N}\left(v_{0}\right) \geq-1$ with equality if and only if all the asymptotic orbits of $v_{0}$ have odd parity, hence $\hat{c}_{N}\left(v_{0}\right) \geq 0$. For components $v_{j, i}^{-}$in lower levels, (2.12) implies $c_{N}\left(v_{j, i}^{-}\right) \geq 0$ unless $v_{j, i}^{-}$is a cover of a trivial cylinder. If on the other hand $v_{j, i}^{-}$covers a trivial cylinder over some orbit $\gamma$, Proposition 2.8 gives $\operatorname{ind}\left(v_{j, i}^{-}\right) \geq 0$, with strict inequality unless $\gamma$ is elliptic or the cover is unbranched. Strict inequality would imply $c_{N}\left(v_{j, i}^{-}\right) \geq 0$ due to (2.10). The scenario $c_{N}\left(v_{j, i}^{-}\right)<0$ can thus happen only if $\gamma$ is elliptic and $\operatorname{ind}\left(v_{j, i}^{-}\right)=0$, in which case (2.10) gives $c_{N}\left(v_{i, j}\right)=-1$ and thus $\hat{c}_{N}\left(v_{i, j}\right) \geq 0$, with strict inequality if $v_{i, j}$ has more than one negative puncture. Using (5.1), we conclude that $\hat{c}\left(v_{0}\right)=0$ and $\hat{c}\left(v_{j, i}^{-}\right)=0$ for every $i, j$, which implies that all components in $u_{\infty}$ having negative normal Chern number have exactly one negative puncture, i.e. they are cylinders. For components in lower levels, this means they are trivial 
cylinders over elliptic Reeb orbits, and no other lower level components can have any negative asymptotic orbits with odd parity since this would imply $\hat{c}_{N}\left(v_{j, i}^{-}\right)>0$. Note that since $u_{\infty}$ is assumed to be stable in the sense of $\mathrm{BEH}^{+}$03], it does not have any levels consisting only of trivial cylinders. It follows that if $u_{\infty}$ has any odd breaking orbits at all, then $v_{0}$ is an index 0 cylinder with $c_{N}\left(v_{0}\right)=-1$ and a negative orbit with odd parity, but all other breaking orbits in the building are even.

Proof of Theorem 5.1. Consider first the case where all breaking orbits have even parity. We know that $v_{0}$ is somewhere injective since $\gamma_{\infty}$ is a simple orbit, but each lower level component $v_{j, i}^{-}$could be a multiple cover, say a $k_{j, i}$-fold cover of a somewhere injective curve $w_{j, i}$. Each $w_{j, i}$ that is not a trivial cylinder satisfies ind $\left(w_{j, i}\right) \geq 1$ due to genericity, so by Proposition 2.9.

$$
\operatorname{ind}\left(v_{j, i}^{-}\right) \geq k_{j, i} \cdot \operatorname{ind}\left(w_{j, i}\right) \geq k_{j, i}
$$

with strict inequality unless the cover is unbranched. Thus if any $k_{j, i}$ is greater than 1 , the fact that $\operatorname{ind}\left(u_{k}\right) \leq 2$ then $\operatorname{implies} \operatorname{ind}\left(v_{j, i}^{-}\right)=k_{j, i}=2$, $v_{j, i}^{-}$is an unbranched double cover of $w_{j, i}$, and all other components in the building must have index 0 . Note that if this happens, $v_{j, i}^{-}$cannot be a plane, as $w_{j, i}$ would then also be a plane and the Riemann-Hurwitz formula precludes the existence of an unbranched cover $\mathbb{C} \rightarrow \mathbb{C}$. But $u_{\infty}$ definitely also has components that are capping planes, which also must have positive index, so this gives a contradiction, proving that every $v_{j, i}^{-}$is either somewhere injective or is a trivial cylinder over an orbit with even parity. Since the curves $u_{k}$ converging to $u_{\infty}$ are all embedded, Corollary 1.17now implies that all breaking orbits are either simple or doubly covered bad orbits. To see that all components are also nicely embedded, we use Proposition 2.11 to write

$$
0 \geq u_{k} * u_{k} \geq v_{0} * v_{0}+\sum_{j=1}^{N} \sum_{i, \ell=1}^{m_{j}} v_{j, i}^{-} * v_{j, \ell}^{-} .
$$

Positivity of intersections together with Prop. 2.10 implies that all terms on the right hand side are nonnegative, hence all of them vanish, including each $v_{j, i}^{-} * v_{j, i}^{-}$. Since $c_{N}\left(v_{j, i}^{-}\right)=c_{N}\left(v_{0}\right)=0$, the adjunction formula (2.15) then gives $\delta_{\text {total }}\left(v_{j, u}^{-}\right)=\delta_{\text {total }}\left(v_{0}\right)=0$, hence all components are nicely embedded.

By Lemma 5.2, we must also consider the case where $v_{0}$ is a cylinder with $c_{N}\left(v_{0}\right)=-1$ whose asymptotic orbits are both odd, while all other breaking orbits are even and all lower level components satisfy $c_{N}\left(v_{j, i}^{-}\right)=0$. The adjunction formula (2.15) implies $v_{0} * v_{0} \geq-1$. For any pair of components $v_{j, i}^{-}$and $v_{j, \ell}^{-}$that are not both trivial cylinders, positivity of intersections gives $v_{j, i}^{-} * v_{j, \ell}^{-} \geq 0$; note that this is guaranteed even if they are (covers of) the same curve since $\mathbb{R}$-invariance allows us to push one of them so that they have only isolated intersections. This trick only fails if both are covers of the same trivial cylinder, but in that case, we know that the underlying Reeb orbit cannot have odd parity, hence Prop. 2.10 applies again to give $v_{j, i}^{-} * v_{j, \ell}^{-} \geq 0$. Now Proposition 2.11 implies

$$
0 \geq u_{k} * u_{k} \geq-1+m(\gamma)
$$


where $\gamma$ is the odd breaking orbit between $v_{0}$ and $v_{1,1}^{-}$and $m(\gamma) \in \mathbb{N}$ denotes its covering multiplicity; we conclude that this orbit must be simply covered. It follows that $v_{1,1}^{-}$is a somewhere injective curve and thus has $\operatorname{ind}\left(v_{1,1}^{-}\right) \geq$ 1. For all curves in levels below this, the absence of odd orbits means that we can again use the arguments of the previous paragraph to rule out multiple covers, and we conclude again that all components in $u_{\infty}$ are somewhere injective except possibly for trivial cylinders. The constraints on multiplicities of the breaking orbits and the conclusion that all nontrivial components are nicely embedded now follow from the same arguments using the local adjunction formula and Proposition 2.11.

To obtain the classification of buildings stated in the theorem, it remains only to add up Fredholm indices, using the fact that ind $\left(v_{0}\right) \geq 0$ and all nontrivial components in lower levels have index at least 1 . The conclusions about parities of Reeb orbits then follow directly from the index formula.

\section{Proofs of the MaIN theorems}

We shall now prove Theorems 1.1 and 1.2 in reverse order.

Proof of Theorem 1.2(2). Assume $(W, d \lambda)$ is a 4-dimensional Liouville cobordism from $(M, \xi)$ to $\left(M_{+}, \xi_{+}\right)$, where $\left(M_{+}, \xi_{+}\right)$is overtwisted, and $\alpha$ is a nondegenerate contact form for $\xi$. After possibly rescaling $\alpha$ by a positive constant, we can arrange $\left.\lambda\right|_{T M}=\alpha$ and $\left.\lambda\right|_{T M_{+}}=\alpha_{+}$, where $\alpha_{+}$is the particular nondegenerate contact form described in 3.3 . Arguing by contradiction, assume $\alpha$ does not admit any unknotted Reeb orbit with Conley-Zehnder index 2 and self-intersection number -1 . By Lemma 2.18, this means that nicely embedded planes with index 2 and simply covered asymptotic orbits cannot exist in $\left(\mathbb{R} \times M, J_{-}\right)$for any $J_{-} \in \mathcal{J}(\alpha)$; we will use this to exclude some of the possible buildings listed in Theorem 5.1 and thus derive a contradiction.

Choose $J \in \mathcal{J}\left(W, d \lambda, \alpha_{+}, \alpha\right)$ to be generic in the interior of $W$ such that its restriction to the negative cylindrical end is a generic element $J_{-} \in \mathcal{J}(\alpha)$ and its restriction to the positive end matches $J_{+} \in \mathcal{J}\left(\alpha_{+}\right)$from $\$ 3.3$. Then the nicely embedded plane $u^{\infty} \in \mathcal{M}\left(J_{+}, \gamma_{\infty}, \emptyset\right)$ constructed in that section gives rise to a 1-parameter family of nicely embedded curves in $\mathcal{M}\left(J, \gamma_{\infty}, \emptyset\right)$, living in the cylindrical end $[0, \infty) \times M_{+} \subset \bar{W}$; we shall refer to these henceforth as the seed curves in $\bar{W}$. They are Fredholm regular by Prop. 2.15, Let

$$
\mathcal{M}^{\text {nice }}(J) \subset \mathcal{M}\left(J, \gamma_{\infty}, \emptyset\right)
$$

denote the set of all nicely embedded planes in $\mathcal{M}\left(J, \gamma_{\infty}, \emptyset\right)$ that belong to the same relative homology class as the seed curves. These all have index 1 , and the uniqueness of curves in $\left(\mathbb{R} \times M_{+}, J_{+}\right)$implies that all of them other than the seed curves intersect the region where $J$ is generic, thus $\mathcal{M}^{\text {nice }}(J)$ is a smooth 1-manifold. Its closure

$$
\overline{\mathcal{M}}^{\text {nice }}(J) \subset \overline{\mathcal{M}}\left(J, \gamma_{\infty}, \emptyset\right)
$$

in the SFT compactification can now be described as follows. If $u_{k} \in$ $\mathcal{M}^{\text {nice }}(J)$ is a sequence converging to a building with a nontrivial upper level, then the uniqueness of curves in $\left(\mathbb{R} \times M_{+}, J_{+}\right)$implies that this building is $\left(u^{\infty} \mid \emptyset\right)$, where $u^{\infty}$ here is an upper level and the main level is empty. 
This can only be the limit if $u_{k}$ consists of seed curves for sufficiently large $k$, so a neighborhood of $\left(u^{\infty} \mid \emptyset\right)$ in $\overline{\mathcal{M}}^{\text {nice }}(J)$ is homeomorphic to the interval $(-1,0]$, with $\left(u^{\infty} \mid \emptyset\right)$ as the boundary point. If the limit is any building with trivial upper levels but a nontrivial lower level, then it is described by Theorem 5.1, and must in fact be a building of Type (I) since all the others in the list have total index 2 . We can thus write the limit as $\left(v_{0} \mid v_{1}^{-}\right)$for an index 0 cylinder $v_{0}$ in $(\bar{W}, J)$ and an index 1 plane $v_{1}^{-}$in $\left(\mathbb{R} \times M, J_{-}\right)$, both nicely embedded. The breaking orbit between these must be a doubly covered bad orbit due to the assumption excluding unknotted orbits. Proposition 2.6 on gluing implies that a neighborhood of $\left(v_{0} \mid v_{1}^{-}\right)$in $\overline{\mathcal{M}}^{\text {nice }}(J)$ is also homeomorphic to $(-1,0]$, with the building forming the boundary point. We've thus shown that each connected component of $\overline{\mathcal{M}}^{\text {nice }}(J)$ has the topology of a compact connected 1-manifold with boundary, so either a circle or a closed interval, and at least one component is of the latter type, namely the one containing the seed curves. We claim in fact that $\overline{\mathcal{M}}^{\text {nice }}(J)$ itself is compact. In light of the above description, this can only fail to be true if $\overline{\mathcal{M}}^{\text {nice }}(J)$ has infinitely many connected components, in which case we can find a sequence of nicely embedded curves $u_{k} \in \mathcal{M}^{\text {nice }}(J)$ all belonging to separate components. But these curves are all homologous and thus satisfy a uniform energy bound, so they have an SFT-convergent subsequence by $\mathrm{BEH}^{+} 03$, whose limit is in $\overline{\mathcal{M}}^{\text {nice }}(J)$ by definition. We conclude that $\overline{\mathcal{M}}^{\text {nice }}(J)$ is a compact 1-manifold with boundary.

The crucial observation is now that since the breaking orbit in $\left(v_{0} \mid v_{1}^{-}\right)$ is doubly covered, there are always exactly two choices of decoration which give two distinct elements of $\overline{\mathcal{M}}\left(J, \gamma_{\infty}, \emptyset\right)$ having the same curves as their main and lower levels. Indeed, these cannot represent equivalent elements of $\overline{\mathcal{M}}\left(J, \gamma_{\infty}, \emptyset\right)$ since the levels are both somewhere injective and thus admit no automorphisms that could change the decoration. Moreover, both buildings can be glued via Prop. 2.6 to produce smooth 1-parameter families of somewhere injective planes in $\mathcal{M}\left(J, \gamma_{\infty}, \emptyset\right)$, which will be nicely embedded since they are homologous to the seed curves, thus both buildings also belong to $\overline{\mathcal{M}}^{\text {nice }}(J)$. With this understood, let

$$
\widehat{\mathcal{M}}^{\text {nice }}(J)=\overline{\mathcal{M}}^{\text {nice }}(J) / \sim,
$$

where the equivalence relation identifies any two buildings that have matching levels but different decorations. Topologically, $\widehat{\mathcal{M}}^{\text {nice }}(J)$ is formed by gluing components of $\overline{\mathcal{M}}^{\text {nice }}(J)$ together along boundary points of the form $\left(v_{0} \mid v_{1}^{-}\right)$, all of which become interior points in $\widehat{\mathcal{M}}^{\text {nice }}(J)$. But exactly one boundary point of $\overline{\mathcal{M}}^{\text {nice }}(J)$ does not belong to a matching pair, namely $\left(u^{\infty} \mid \emptyset\right)$, thus $\widehat{\mathcal{M}}^{\text {nice }}(J)$ is homeomorphic to a compact 1-manifold with one boundary point, giving a contradiction.

Proof of Theorem 1.2(1). Assume $(M, \xi)$ is a reducible tight contact 3-manifold, and fix the contact form $\alpha_{+}$and almost complex structure $J_{+} \in \mathcal{J}\left(\alpha_{+}\right)$ described in 3.2 , so there is a homotopically nontrivial 2-sphere $S \subset M$ containing a simple nondegenerate Reeb orbit $\gamma_{\infty}: S^{1} \rightarrow M$ that divides $S$ into hemispheres $S_{ \pm}=u_{M}^{ \pm}(\mathbb{C})$ that are each images of nicely embedded 
$J_{+}$-holomorphic index 1 planes

$$
u^{ \pm}=\left(u_{\mathbb{R}}^{ \pm}, u_{M}^{ \pm}\right): \mathbb{C} \rightarrow \mathbb{R} \times M .
$$

By Lemma 3.5, these are the only $J_{+}$-holomorphic curves up to parametrization and $\mathbb{R}$-translation that have a single positive puncture asymptotic to $\gamma_{\infty}$ (and arbitrary negative punctures).

Now pick an arbitrary nondegenerate contact form $\alpha$ on $(M, \xi)$, and suppose it admits no unknotted Reeb orbit with Conley-Zehnder index 2 and self-linking number -1 . We are free to rescale $\alpha_{+}$so that $\alpha_{+}=e^{f} \alpha$ for some $f: M \rightarrow(0, \infty)$ without loss of generality. There is then a Liouville cobordism $(W, d \lambda)$ with

$$
W=\{(r, x) \in \mathbb{R} \times M \mid 0 \leq r \leq f(x), x \in M\}, \quad \lambda=e^{r} \alpha,
$$

which inherits the contact form $\alpha$ on its concave boundary $M_{-}:=\{0\} \times M$ and $\alpha_{+}$on its convex boundary $M_{+}:=\{(f(x), x) \mid x \in M\}$. Choose a generic $J \in \mathcal{J}\left(W, d \lambda, \alpha_{+}, \alpha\right)$ that matches $J_{+}$in the positive end and has a generic restriction $J_{-} \in \mathcal{J}(\alpha)$ to the negative end. The $\mathbb{R}$-translations of $u^{+}$and $u^{-}$then give rise to a disjoint pair of 1-parameter families of nicely embedded seed curves in the completion $\bar{W}$, living in the positive cylindrical end.

As in the overtwisted case, we consider the space $\mathcal{M}^{\text {nice }}(J) \subset \mathcal{M}\left(J, \gamma_{\infty}, \emptyset\right)$ of nicely embedded planes that are in the same relative homology class with either family of seed curves, and its closure $\overline{\mathcal{M}}^{\text {nice }}(J)$ in the SFT compactification. Then $\overline{\mathcal{M}}^{\text {nice }}(J)$ contains the two elements $\left(u^{ \pm} \mid \emptyset\right)$ with empty main levels, plus (by Theorem 5.1) buildings of the form $\left(v_{0} \mid v_{1}^{-}\right)$with a nicely embedded index 1 plane $v_{1}^{-}$in the lower level and breaking orbits that are doubly covered. The same argument as in the overtwisted case proves that $\overline{\mathcal{M}}^{\text {nice }}(J)$ has the topology of a compact 1-manifold with boundary, where boundary points of the form $\left(v_{0} \mid v_{1}^{-}\right)$come in matching pairs with the same levels but different decorations due to the doubly covered breaking orbit. The space

$$
\widehat{\mathcal{M}}^{\text {nice }}(J)=\overline{\mathcal{M}}^{\text {nice }}(J) / \sim
$$

defined by identifying matching pairs is thus a compact 1-manifold with exactly two boundary points, the two buildings $\left(u^{+} \mid \emptyset\right)$ and $\left(u^{-} \mid \emptyset\right)$. It follows that these two buildings belong to the same connected component in $\widehat{\mathcal{M}}^{\text {nice }}(J)$, and the images of the curves or buildings in this component under the projection $\mathbb{R} \times M \rightarrow M$ then give a continuous 1-parameter family of disks with fixed boundary $\gamma_{\infty}$ forming a homotopy from $S_{+}$to $S_{-}$in $M$. This is a contradiction since $[S] \neq 0 \in \pi_{2}(M)$.

Proof of Theorem 1.1. Recall from $\$ 3.1$ the definitions of the standard contact form $\alpha_{\text {std }}$ and the almost complex structure $J_{\text {std }} \in \mathcal{J}\left(\alpha_{\text {std }}\right)$. Given a Liouville cobordism $(W, d \lambda)$ from some contact manifold $(M, \xi)$ to $\left(S^{3}, \xi_{\text {std }}\right)$ with $\left.\lambda\right|_{T S^{3}}=\alpha_{\text {std }}$ and a nondegenerate contact form $\left.\lambda\right|_{T M}=\alpha$ on $(M, \xi)$, one can choose a generic $J \in \mathcal{J}\left(W, d \lambda, \alpha_{\text {std }}, \alpha\right)$ that matches $J_{\text {std }}$ in the positive cylindrical end and has a generic restriction $J_{-} \in \mathcal{J}(\alpha)$ to the negative cylindrical end. The seed curves $u_{w}$ constructed in 33.1 that are 
contained in $[0, \infty) \times S^{3}$ can then equally well be regarded as nicely embedded $J$-holomorphic planes in the completed cobordism $\bar{W}$ with images in the positive cylindrical end. Recall that by Lemma 3.3, every curve in $\mathbb{R} \times S^{3}$ with one positive puncture asymptotic to the particular orbit $\gamma_{\infty}$ (and arbitrary negative punctures) is one of these planes.

Let $\mathcal{M}_{1}^{\text {nice }}(J) \subset \mathcal{M}_{1}\left(J, \gamma_{\infty}, \emptyset\right)$ denote the set of all nicely embedded planes in the same relative homology class as the seed curves, carrying the extra data of one marked point, and denote its closure in the SFT compactification by $\overline{\mathcal{M}}_{1}^{\text {nice }}(J) \subset \overline{\mathcal{M}}_{1}\left(J, \gamma_{\infty}, \emptyset\right)$. All curves in $\mathcal{M}_{1}^{\text {nice }}(J)$ have index 2 and are Fredholm regular, so $\mathcal{M}_{1}^{\text {nice }}(J)$ is a smooth 4-dimensional manifold. As a consequence of Lemma 3.3, all buildings in $\overline{\mathcal{M}}_{1}^{\text {nice }}(J)$ with nontrivial upper levels are of the form $\left(u_{w} \mid \emptyset\right)$ with $u_{w}$ a seed curve and the main level empty. All other buildings in $\overline{\mathcal{M}}^{\text {nice }}(J)$ are described by Theorem 5.1.

We will now show that if $\alpha$ admits no unknotted Reeb orbits with selflinking number -1 and Conley-Zehnder index 2 , then it must admit one with Conley-Zehnder index 3. To this end, choose points $p_{+} \in S^{3}$ and $p_{-} \in M$, and a 1-dimensional submanifold

$$
\ell(\mathbb{R}) \subset \bar{W}
$$

defined via a smooth proper embedding $\ell: \mathbb{R} \hookrightarrow \bar{W}$ such that $\ell(t)=\left(t, p_{+}\right) \in$ $[0, \infty) \times S^{3}$ for all $t>0$ sufficiently large and $\ell(t)=(t, f(t)) \in(-\infty, 0] \times$ $M$ for $t<0$ sufficiently small, with $\lim _{t \rightarrow-\infty} f(t)=p_{-}$. After generic perturbations of both $p_{-}$and $\ell$ away from $+\infty$, we are free to assume:

(1) $\ell$ is transverse to the evaluation map on the moduli space of all somewhere injective $J$-holomorphic curves in $\bar{W}$ that are not fully contained in the positive end;

(2) $p_{-}$is not contained in any closed Reeb orbit;

(3) $\mathbb{R} \times\left\{p_{-}\right\}$is transverse to the evaluation map on the moduli space of all somewhere injective $J_{-}$-holomorphic curves in $\mathbb{R} \times M$.

Now consider the 1-dimensional submanifold

$$
\mathcal{M}_{\ell}^{\text {nice }}(J)=\mathrm{ev}^{-1}(\ell(\mathbb{R})) \subset \mathcal{M}_{1}^{\text {nice }}(J),
$$

and for convenience define

$$
\overline{\mathcal{M}}_{\ell}^{\text {nice }}(J) \subset \overline{\mathcal{M}}_{1}^{\text {nice }}(J)
$$

to be the set of all buildings in its SFT-closure that have the marked point in the main level. The evaluation map then restricts to

$$
\overline{\mathcal{M}}_{\ell}^{\text {nice }}(J) \stackrel{\text { ev }}{\longrightarrow} \ell(\mathbb{R}),
$$

and the image of this map clearly contains an interval of the form $\ell\left(\left[t_{0}, \infty\right)\right)$ due to the seed curves. The goal of the next two paragraphs is to show that this map is in fact surjective onto $\ell(\mathbb{R})$.

Observe first that the restriction of ev to $\mathcal{M}_{\ell}^{\text {nice }}(J)$ is an open map due to Prop. 2.16. We claim moreover that any building $u \in \overline{\mathcal{M}}_{\ell}^{\text {nice }}(J)$ with ev $(u)=\ell\left(t_{0}\right)$ has a neighborhood in $\overline{\mathcal{M}}_{\ell}^{\text {nice }}(J)$ whose image under ev contains $\ell\left(\left[t_{0}, t_{0}+\epsilon\right)\right)$ or $\ell\left(\left(t_{0}-\epsilon, t_{0}\right]\right)$ for sufficiently small $\epsilon>0$. To see this, note first that unless $u$ is a smooth curve, it is necessarily one of Types (II) though (VI) on the list in Theorem 5.1, but our genericity assumptions impose further 
restrictions: since $\ell$ intersects the evaluation map transversely, the main level of the building must have index at least 1, excluding all options other than Type (III). We can thus write $u=\left(v_{0} \mid v_{1}^{-}\right)$for an index 1 cylinder $v_{0} \in$ $\mathcal{M}_{1}\left(J, \gamma_{\infty}, \gamma\right)$ with a marked point, and an index 1 plane $v_{1}^{-} \in \mathcal{M}\left(J_{-}, \gamma, \emptyset\right)$, both nicely embedded. Here the lower level $v_{1}^{-}$represents an isolated element in $\mathcal{M}\left(J_{-}, \gamma, \emptyset\right) / \mathbb{R}$, while $v_{0}$ has a neighborhood $\mathcal{V}_{0} \subset \mathcal{M}_{1}\left(J, \gamma_{\infty}, \gamma\right)$ that is a smooth 3-manifold, and by choosing this neighborhood sufficiently small, we can assume that

$$
\mathcal{V}_{0} \stackrel{e v}{\longrightarrow} \bar{W}
$$

has only one intersection with $\ell(\mathbb{R})$, namely at $v_{0}$, and it is transverse. Proposition 2.6 now gives a smooth gluing map

$$
\Psi:[0, \infty) \times \mathcal{V}_{0} \hookrightarrow \mathcal{M}_{1}(J, \gamma, \emptyset)
$$

whose image contains all smooth curves close to $\left(v_{0} \mid v_{1}^{-}\right)$in the SFT topology, and all of these belong to $\mathcal{M}_{1}^{\text {nice }}(J)$. The maps

$$
\{R\} \times \mathcal{V}_{0} \rightarrow \bar{W}: v \mapsto \operatorname{ev}(\Psi(R, v))
$$

can then be assumed to converge uniformly to (6.1) as $R \rightarrow \infty$, implying that their algebraic count of intersections with $\ell(\mathbb{R})$ is 1 for all $R>0$ sufficiently large. Choosing $R_{0}>0$ large and generic, the subset

$$
\mathcal{U}_{\ell}:=\left\{(R, v) \in\left[R_{0}, \infty\right) \times \mathcal{V}_{0} \mid \operatorname{ev}(\Psi(R, v)) \in \ell(\mathbb{R})\right\}
$$

is then a smooth and properly embedded 1-manifold that intersects $\left\{R_{0}\right\} \times$ $\mathcal{V}_{0}$ transversely at its boundary $\partial \mathcal{U}_{\ell}$, which is a finite set of points. This 1-manifold must have at least one noncompact component, otherwise the algebraic count of points in $\partial \mathcal{U}_{\ell}$ could not be 1 , hence there exists a smooth path $\mathcal{U}_{\ell}^{0} \subset\left[R_{0}, \infty\right) \times \mathcal{V}_{0}$ whose image under $\Psi$ is a smooth family of curves $u_{t} \in \mathcal{M}_{\ell}^{\text {nice }}(J)$ with $t \in[0, \infty)$ such that

$$
u_{t} \rightarrow\left(v_{0} \mid v_{1}^{-}\right) \quad \text { as } \quad t \rightarrow \infty .
$$

The image of this path under ev necessarily contains $\ell\left(\left(t_{0}, t_{0}+\epsilon\right)\right)$ or $\ell\left(\left(t_{0}-\right.\right.$ $\left.\epsilon, t_{0}\right)$ ) as claimed.

Now, given the lack of unknotted orbits with Conley-Zehnder index 2, the breaking orbit in the building $\left(v_{0} \mid v_{1}^{-}\right)$of the previous paragraph must be doubly covered, so that building has a twin obtained by keeping the same levels but changing the decoration, and the fact that both levels are somewhere injective implies that the two buildings are not equivalent in $\overline{\mathcal{M}}_{1}\left(J, \gamma_{\infty}, \emptyset\right)$. Thus the twin building can also be glued using Prop. 2.6 and produces a nearby family of curves in $\mathcal{M}_{1}^{\text {nice }}(J)$, some of which satisfy $\mathrm{ev}(u) \in \ell(\mathbb{R})$ and whose images under ev again cover an interval of the form $\ell\left(\left(t_{0}, t_{0}+\epsilon\right)\right)$ or $\ell\left(\left(t_{0}-\epsilon, t_{0}\right)\right)$. But since no two curves in $\mathcal{M}_{1}^{\text {nice }}(J)$ can intersect, the curves in $\mathcal{M}_{\ell}^{\text {nice }}(J)$ obtained by gluing the same curves $v_{0}$ and $v_{1}^{-}$with two distinct decorations necessarily cover two disjoint intervals, so that the entirety of $\ell\left(\left(t_{0}-\epsilon, t_{0}+\epsilon\right)\right)$ is necessarily in the image of $\overline{\mathcal{M}}_{\ell}^{\text {nice }}(J)$ for sufficiently small $\epsilon>0$. This proves that that image is an open subset of $\ell(\mathbb{R})$, hence it is all of $\ell(\mathbb{R})$.

With this established, we can now find a sequence $u_{k} \in \mathcal{M}_{\ell}^{\text {nice }}(J)$ with $\operatorname{ev}\left(u_{k}\right)=\ell\left(t_{k}\right)$ for some sequence $t_{k} \rightarrow-\infty$, and a subsequence of $u_{k}$ must 
again converge to one of the buildings listed in Theorem 5.1, but this time with the marked point ending up in a lower level and mapping to $\mathbb{R} \times\left\{p_{-}\right\}$. Since $p_{-}$is not in the image of any Reeb orbit, the marked point in the limit does not lie on a trivial cylinder. Transversality of $\mathbb{R} \times\left\{p_{-}\right\}$to the evaluation map thus implies that the component with the marked point must have index at least 2, which rules out all options in the list other than Type (II): $u_{k}$ has a subsequence covergent to $\left(v_{0} \mid v_{1}^{-}\right)$where $v_{1}^{-}$is a nicely embedded plane in $(\mathbb{R} \times M, J)$ with index 2 and an asymptotic orbit with odd parity, which is therefore simply covered. This is the promised unknotted orbit with self-linking number -1 and Conley-Zehnder index 3 .

\section{Appendix A. Liouville cobordisms from exaCt Lagrangian CAPS}

In this appendix, we provide the details behind Example 1.6. using a general construction that was explained to us by Emmy Murphy.

Proposition A.1. Suppose $(M, \xi)$ is a closed contact manifold of dimension $2 n-1 \geq 3, \Lambda \subset M$ is a closed Legendrian submanifold and $L \subset[1, \infty) \times M$ is an exact Lagrangian cap for $\Lambda$. Then $L$ has an open neighbourhood $\mathcal{U}_{L} \subset$ $[1, \infty) \times M$ such that, after smoothing corners,

$$
W_{-}:=([0,1] \times M) \cup \overline{\mathcal{U}}_{L}
$$

admits the structure of a Weinstein cobordism from $(M, \xi)$ to some contact manifold $\left(M^{\prime}, \xi^{\prime}\right)$, and for suitably large constants $T>1$,

$$
W_{+}:=([1, T] \times M) \backslash \mathcal{U}_{L}
$$

is a Liouville cobordism from $\left(M^{\prime}, \xi^{\prime}\right)$ to $(M, \xi)$.

Proof. Being an exact Lagrangian cap means that for some choice of contact form $\alpha$ on $(M, \xi)$ and some constant $T>1$, the trivial Liouville cobordism

$$
(Z, d \lambda):=\left([1, T] \times M, d\left(e^{r} \alpha\right)\right)
$$

contains $L$ as a compact and properly embedded Lagrangian submanifold with $\partial L=\{1\} \times \Lambda$, such that the Liouville vector field $\partial_{t}$ is tangent to $L$ near $\partial L$ and

$$
\left.\lambda\right|_{T L}=d g
$$

for some smooth function $g: L \rightarrow \mathbb{R}$. Note that since $\Lambda$ is Legendrian and $\lambda$ annihilates its dual Liouville vector field, $g$ must be constant near $\partial L$; we shall assume without loss of generality that it vanishes there. By a combination of the Lagrangian and Legendrian neighbourhood theorems, $L$ has a symplectic neighbourhood $\left(\mathcal{U}_{L}, d \lambda\right)$ whose closure $\overline{\mathcal{U}}_{L}$ is symplectomorphic to the unit disk bundle in $\mathbb{D} T^{*} L \subset T^{*} L$ for some choice of Riemannian metric on $L$. Note that this disk bundle has boundary and corners, its boundary consisting of two smooth faces,

$$
\partial_{-} \overline{\mathcal{U}}_{L}:=\left.\mathbb{D} T^{*} L\right|_{\partial L} \quad \text { and } \quad \partial_{+} \overline{\mathcal{U}}_{L}:=S T^{*} L,
$$

where $S T^{*} L$ is the unit cotangent bundle. We shall write points in $T^{*} L$ as $(q, p)$ for $q \in L$ and $p \in T_{q}^{*} L$, and use the metric and its induced Levi-Civita connection to identify $T_{(q, p)}\left(T^{*} L\right)$ with $T_{q} L \oplus T_{q}^{*} L=T_{q} L \oplus T_{q} L$, where the first splitting comes from the horizontal-vertical decomposition given by the 
connection, and the second uses the isomorphism $T_{q} L=T_{q}^{*} L$ determined by the metric. The canonical Liouville form $\lambda_{0}$ on $T^{*} L$ can then be written as

$$
\lambda_{0}=-d F_{0} \circ J
$$

where $F_{0}(q, p)=\frac{1}{2}|p|^{2}$ and $J$ is the compatible almost complex structure on $T^{*} L$ that acts on $T_{(q, p)}\left(T^{*} L\right)=T_{q} L \oplus T_{q} L$ as $\left(\begin{array}{cc}0 & \mathbb{1} \\ -\mathbb{1} & 0\end{array}\right)$. In particular, $F_{0}$ is a $J$-convex function, and therefore so is

$$
F_{\epsilon}(q, p):=\epsilon f(q)+\frac{1}{2}|p|^{2}
$$

for any smooth function $f: L \rightarrow \mathbb{R}$ if $\epsilon>0$ is sufficiently small. Setting $\lambda_{\epsilon}:=-d F_{\epsilon} \circ J, d \lambda_{\epsilon}$ is then a symplectic form isotopic to $d \lambda_{0}$ on a suitable neighbourhood of the zero-section $L$, and since the antipodal map $(q, p) \mapsto$ $(q,-p)$ is $J$-antiholomorphic but preserves $F_{\epsilon}$, it also preserves the Liouville vector field $V_{\epsilon}$ dual to $\lambda_{\epsilon}$, proving that $V_{\epsilon}$ is tangent to $L$.

Now choose $f: L \rightarrow \mathbb{R}$ in this construction to be a Morse function that is constant with inward-pointing gradient along $\partial L$. After possibly shrinking the neighbourhood $\overline{\mathcal{U}}_{L} \cong \mathbb{D} T^{*} L$ of $L$, we can then assume that $V_{\epsilon}$ points transversely inward at $\partial_{-} \overline{\mathcal{U}}_{L}$ and transversely outward at $\partial_{+} \overline{\mathcal{U}}_{L}$. Since the Liouville field of $(Z, d \lambda)$ is also tangent to $L$ near $\partial L$ and points inward at $\{1\} \times M \subset \partial Z$ (see Figure 1), we can now assume after an isotopy of $\overline{\mathcal{U}}_{L}$ that the two Liouville fields match near $\partial_{-} \overline{\mathcal{U}}_{L}$, meaning $\lambda=\lambda_{\epsilon}$ on that region. We can therefore use $\lambda_{\epsilon}$ to extend $\lambda$ from $[0,1] \times M$ over $W_{-}$so that the dual Liouville vector field remains gradient like, making $W_{-}$a Weinstein cobordism from $(M, \xi)$ to the new contact manifold $\left(M^{\prime}, \xi^{\prime}\right)$, obtained by removing a neighbourhood of $\Lambda$ from $\{1\} \times M$ and replacing it with $S T^{*} L$.

It is also immediate from the above construction that $W_{+}$is a strong symplectic cobordism from $\left(M^{\prime}, \xi^{\prime}\right)$ to $(M, \xi)$, and the exactness of the cobordism follows from the fact that $L$ is an exact Lagrangian. Indeed, let $\dot{\mathcal{U}}_{L}:=\left.\overline{\mathcal{U}}_{L} \backslash \partial_{-} \overline{\mathcal{U}}_{L} \cong \mathbb{D} T^{*} L\right|_{L}$. Since $\lambda$ and $\lambda_{\epsilon}$ match near $\partial_{-} \overline{\mathcal{U}}_{L}$ and are both primitives of the same symplectic form, $\lambda-\lambda_{\epsilon}$ represents an element of the compactly supported de Rham cohomology $H_{c}^{1}\left(\dot{\mathcal{U}}_{L}\right)$, which is isomorphic to $H_{c}^{1}(\stackrel{\circ}{L})$. But under restriction to $L, \lambda_{\epsilon}$ vanishes and $\lambda$ is exact, so this cohomology class is zero, implying $\lambda=\lambda_{\epsilon}+d h$ on $\mathcal{U}_{L}$ for some smooth function $h: \mathcal{U}_{L} \rightarrow \mathbb{R}$ that vanishes near $\partial_{-} \overline{\mathcal{U}}_{L}$. By multiplying $h$ with a suitable cutoff function, we can then find a Liouville form on $W_{+}$that matches $\lambda_{\epsilon}$ near $L$ and matches $\lambda$ outside a neighbourhood of $L$.

Remark A.2. If $W$ is a subcritical Weinstein filling of $(M, \xi)$, then the Weinstein filling of $\left(M^{\prime}, \xi^{\prime}\right)$ obtained by stacking $W_{-}$on top of $W$ is never subcritical. To see this, note that the Morse function $f: L \rightarrow \mathbb{R}$ in the above proof can always be chosen to have exactly one critical point of index $n$, in which case $F_{\epsilon}$ also has exactly one critical point of index $n$. If $W$ is subcritical, this produces a handle decomposition of $W \cup_{M} W_{-}$that includes exactly one critical handle, so $H_{n}\left(W \cup_{M} W_{-}\right) \neq 0$.

\section{REFERENCES}

[AH09] P. Albers and H. Hofer, On the Weinstein conjecture in higher dimensions, Comment. Math. Helv. 84 (2009), no. 2, 429-436. 
[Bou02] F. Bourgeois, A Morse-Bott approach to contact homology, Ph.D. Thesis, Stanford University, 2002.

$\left[\mathrm{BEH}^{+} 03\right]$ F. Bourgeois, Y. Eliashberg, H. Hofer, K. Wysocki, and E. Zehnder, Compactness results in symplectic field theory, Geom. Topol. 7 (2003), 799-888.

[CE12] K. Cieliebak and Y. Eliashberg, From Stein to Weinstein and back: symplectic geometry of affine complex manifolds, American Mathematical Society Colloquium Publications, vol. 59, American Mathematical Society, Providence, RI, 2012.

[CO18] K. Cieliebak and A. Oancea, Symplectic homology and the Eilenberg-Steenrod axioms, Algebr. Geom. Topol. 18 (2018), no. 4, 1953-2130. Appendix written jointly with Peter Albers.

[Co197] V. Colin, Chirurgies d'indice un et isotopies de sphères dans les variétés de contact tendues, C. R. Acad. Sci. Paris Sér. I Math. 324 (1997), no. 6, 659663 (French, with English and French summaries).

[Dra04] D. L. Dragnev, Fredholm theory and transversality for noncompact pseudoholomorphic maps in symplectizations, Comm. Pure Appl. Math. 57 (2004), no. 6, $726-763$

[Eli90] Y. Eliashberg, Filling by holomorphic discs and its applications, Geometry of low-dimensional manifolds, 2 (Durham, 1989), London Math. Soc. Lecture Note Ser., vol. 151, Cambridge Univ. Press, Cambridge, 1990, pp. 45-67.

[EGH00] Y. Eliashberg, A. Givental, and H. Hofer, Introduction to symplectic field theory, Geom. Funct. Anal., Special Volume (2000), 560-673.

[EM13] Y. Eliashberg and E. Murphy, Lagrangian caps, Geom. Funct. Anal. 23 (2013), no. 5, 1483-1514.

[EH02] J. B. Etnyre and K. Honda, On symplectic cobordisms, Math. Ann. 323 (2002), no. 1, 31-39.

[FS18] J. W. Fish and R. Siefring, Connected sums and finite energy foliations I: Contact connected sums, J. Symplectic Geom. 16 (2018), no. 6, 1639-1748.

[Gay06] D. T. Gay, Four-dimensional symplectic cobordisms containing three-handles, Geom. Topol. 10 (2006), 1749-1759.

[Gei08] H. Geiges, An introduction to contact topology, Cambridge Studies in Advanced Mathematics, vol. 109, Cambridge University Press, Cambridge, 2008.

[GZ12] H. Geiges and K. Zehmisch, Symplectic cobordisms and the strong Weinstein conjecture, Math. Proc. Cambridge Philos. Soc. 153 (2012), no. 2, 261-279.

[GZ13a] _ How to recognize a 4-ball when you see one, Münster J. Math. 6 (2013), $525-554$.

[GZ13b] - Erratum to: How to recognize a 4-ball when you see one [MR 3148221], Münster J. Math. 6 (2013), 555-556.

[GZ16] - The Weinstein conjecture for connected sums, Int. Math. Res. Not. 2 (2016), 325-342.

[GNW16] P. Ghiggini, K. Niederkrüger, and C. Wendl, Subcritical contact surgeries and the topology of symplectic fillings, J. Éc. polytech. Math. 3 (2016), 163-208.

[Gro85] M. Gromov, Pseudoholomorphic curves in symplectic manifolds, Invent. Math. 82 (1985), no. 2, 307-347.

[Hat] A. Hatcher, Notes on Basic 3-Manifold Topology. Unpublished manuscript, available at https://www. math. cornell.edu/ hatcher/3M/3Mdownloads.html.

[Hof93] H. Hofer, Pseudoholomorphic curves in symplectizations with applications to the Weinstein conjecture in dimension three, Invent. Math. 114 (1993), no. 3, $515-563$.

[HWZ95] H. Hofer, K. Wysocki, and E. Zehnder, Properties of pseudo-holomorphic curves in symplectisations. II. Embedding controls and algebraic invariants, Geom. Funct. Anal. 5 (1995), no. 2, 270-328.

[HWZ96a] _ Properties of pseudoholomorphic curves in symplectisations. I. Asymptotics, Ann. Inst. H. Poincaré Anal. Non Linéaire 13 (1996), no. 3, 337-379. 
[HWZ96b] _ Properties of pseudoholomorphic curves in symplectisations. IV. Asymptotics with degeneracies, Contact and symplectic geometry (Cambridge, 1994), 1996, pp. 78-117.

[HWZ96c] — Unknotted periodic orbits for Reeb flows on the three-sphere, Topol. Methods Nonlinear Anal. 7 (1996), no. 2, 219-244.

[HWZ99] - Properties of pseudoholomorphic curves in symplectizations. III. Fredholm theory, Topics in nonlinear analysis, 1999, pp. 381-475.

[HWZ03] _ Finite energy foliations of tight three-spheres and Hamiltonian dynamics, Ann. of Math. (2) 157 (2003), no. 1, 125-255.

[HZ94] H. Hofer and E. Zehnder, Symplectic invariants and Hamiltonian dynamics, Birkhäuser Verlag, Basel, 1994.

[Hut02] M. Hutchings, An index inequality for embedded pseudoholomorphic curves in symplectizations, J. Eur. Math. Soc. (JEMS) 4 (2002), no. 4, 313-361.

[Lin16] F. Lin, Exact Lagrangian caps of Legendrian knots, J. Symplectic Geom. 14 (2016), no. 1, 269-295.

[MS04] D. McDuff and D. Salamon, J-holomorphic curves and symplectic topology, American Mathematical Society, Providence, RI, 2004.

[MW95] M. J. Micallef and B. White, The structure of branch points in minimal surfaces and in pseudoholomorphic curves, Ann. of Math. (2) 141 (1995), no. 1, 35-85.

[Mor03] E. Mora, Pseudoholomorphic cylinders in symplectisations, Ph.D. Thesis, New York University, 2003.

[MS18] E. Murphy and K. Siegel, Subflexible symplectic manifolds, Geom. Topol. 22 (2018), no. 4, 2367-2401.

[Nel13] J. Nelson, Applications of automatic transversality in contact homology, Ph.D. Thesis, University of Wisconsin at Madison, 2013.

[Nel15] _ Automatic transversality in contact homology I: regularity, Abh. Math. Semin. Univ. Hambg. 85 (2015), no. 2, 125-179.

[Nie06] K. Niederkrüger, The plastikstufe - a generalization of the overtwisted disk to higher dimensions, Algebr. Geom. Topol. 6 (2006), 2473-2508.

[NR11] K. Niederkrüger and A. Rechtman, The Weinstein conjecture in the presence of submanifolds having a Legendrian foliation, J. Topol. Anal. 3 (2011), no. 4, 405-421.

[Sie08] R. Siefring, Relative asymptotic behavior of pseudoholomorphic half-cylinders, Comm. Pure Appl. Math. 61 (2008), no. 12, 1631-1684.

[Sie11] _ Intersection theory of punctured pseudoholomorphic curves, Geom. Topol. 15 (2011), 2351-2457.

[Wan15] A. Wand, Tightness is preserved by Legendrian surgery, Ann. of Math. (2) 182 (2015), no. 2, 723-738.

[Wen10a] C. Wendl, Compactness for embedded pseudoholomorphic curves in 3manifolds, J. Eur. Math. Soc. (JEMS) 12 (2010), no. 2, 313-342.

[Wen10b] _ Automatic transversality and orbifolds of punctured holomorphic curves in dimension four, Comment. Math. Helv. 85 (2010), no. 2, 347-407.

[Wen13a] _ A hierarchy of local symplectic filling obstructions for contact 3manifolds, Duke Math. J. 162 (2013), no. 12, 2197-2283.

[Wen13b] _ Non-exact symplectic cobordisms between contact 3-manifolds, J. Differential Geom. 95 (2013), no. 1, 121-182.

[Wena] , A biased survey on symplectic fillings, part 4 (cobordisms and flexibility). Blog post, available at https://symplecticfieldtheorist.wordpress.com/2014/11/17/

[Wenb] - Some good news about the forgetful map in SFT. Blog post, available at https://symplecticfieldtheorist.wordpress.com/2015/07/16/

[Wenc] , Lectures on holomorphic curves in symplectic and contact geometry. Preprint arXiv:1011.1690

[Wend] Contact 3-manifolds, holomorphic curves and intersection theory. Preprint arXiv:1706.05540, to appear in Cambridge University Press.

[Wene] — Lectures on Symplectic Field Theory. Preprint arXiv:1612.01009 to appear in EMS Series of Lectures in Mathematics. 
[Yau06] M.-L. Yau, Vanishing of the contact homology of overtwisted contact 3manifolds, Bull. Inst. Math. Acad. Sin. (N.S.) 1 (2006), no. 2, 211-229. With an appendix by Yakov Eliashberg.

Department of Mathematics, University College London, Gower Street, London WC1E 6BT, United Kingdom

E-mail address: a.cioba.12@ucl.ac.uk

Institut für Mathematik, Humboldt-Universität ZU Berlin, Unter Den LinDEN 6, 10099 BerLin, Germany

E-mail address: wendl@math.hu-berlin.de 